Aus der Poliklinik für Zahnärztliche Prothetik

(Prof. Dr. med. dent. R. Bürgers)

im Zentrum Zahn-, Mund- und Kieferheilkunde

der Medizinischen Fakultät der Universität Göttingen

\title{
Untersuchung zum Mundgesundheitszustand und zu Kiefergelenksbefunden bei Kindern und Jugendlichen mit juveniler idiopathischer Arthritis
}

Ergebnisse einer monozentrischen klinischen Querschnittsstudie

\author{
INAUGURAL-DISSERTATION \\ zur Erlangung des Doktorgrades \\ für Zahnheilkunde \\ der Medizinischen Fakultät der \\ Georg-August-Universität zu Göttingen
}

vorgelegt von

Robin Hoffmann

aus

Karlsruhe

Göttingen 2019 
Dekan:

Referent/in

Ko-Referent/in:
Prof. Dr. med. W. Brück

PD Dr. S. Rinke, M.Sc., M.Sc.

PD Dr. Steffi Dreha-Kulaczewski

Datum der mündlichen Prüfung: 19.01.2021 
Hiermit erkläre ich, die Dissertation mit dem Titel "Untersuchung zum Mundgesundheitszustand und zu Kiefergelenksbefunden bei Kindern- und Jugendlichen mit juveniler idiopathischer Arthritis - Ergebnisse einer monozentrischen klinischen Querschnittsstudie" eigenständig angefertigt und keine anderen als die von mir angegebenen Quellen und Hilfsmittel verwendet zu haben.

Göttingen, den

(Unterschrift) 


\section{Inhaltsverzeichnis}

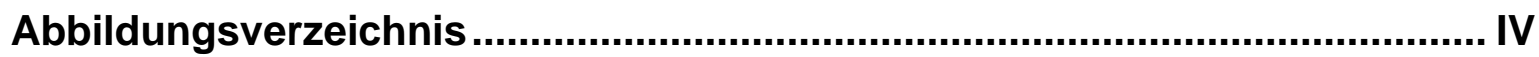

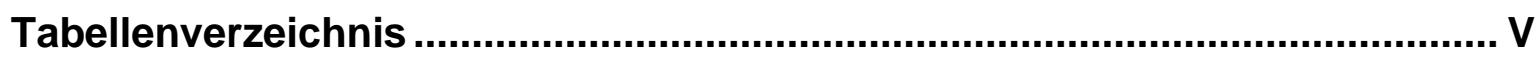

Abkürzungsverzeichnis ............................................................................ VII

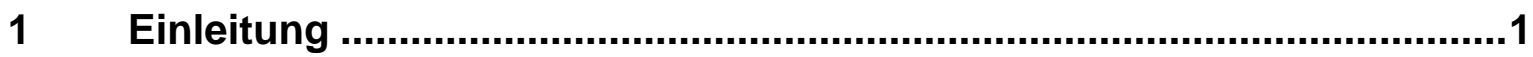

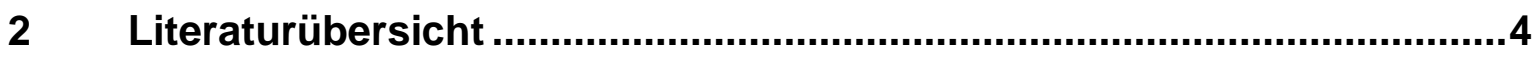

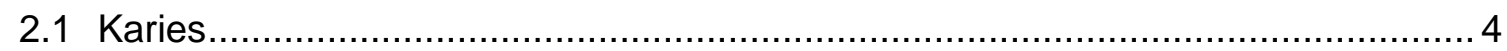

2.1.1 Definition und Epidemiologie ............................................................ 4

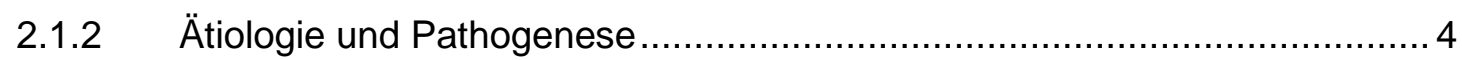

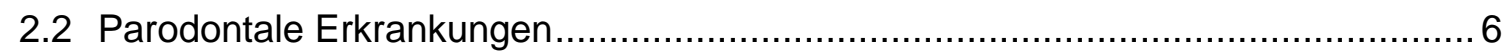

2.2.1 Definition und Epidemiologie ………………...................................

2.2.2 Ätiologie und Pathogenese ...................................................................

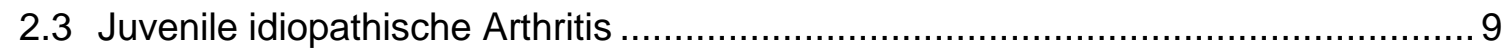

2.3.1 Definition und Epidemiologie ……………….....................................

2.3.2 Ätiologie und Pathogenese ................................................................ 11

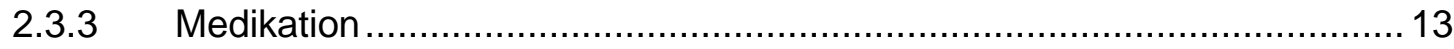

2.3.4 Symptome und Funktionseinschränkungen bei JIA ……........................ 16

2.4 Zusammenhänge zwischen der JIA und oralen Erkrankungen ............................ 19

$3 \quad$ Material und Methode ............................................................................23

3.1 Studientyp

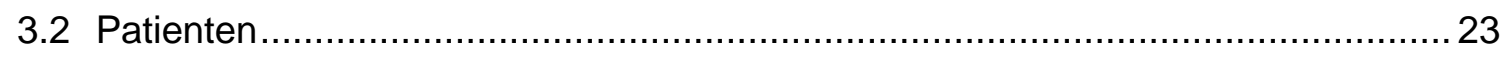

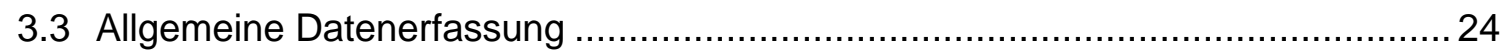

3.3.1 Anamnese- und Funktionsfragebogen .............................................. 25

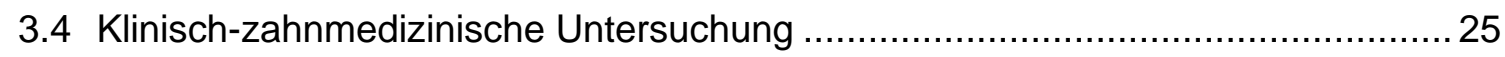

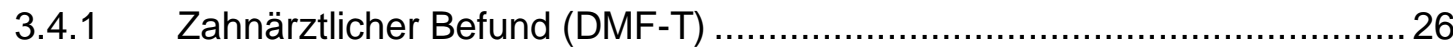

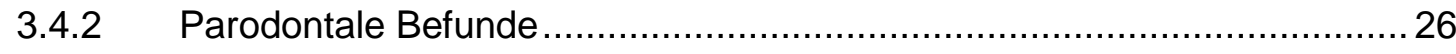

3.4.3 Funktionsdiagnostik (RDC/TMD) nach Dworkin und LeResche (1992) .... 28

3.4.4 Auswertung konsiliarischer Röntgenbilder .................................................. 31

3.5 Datenverarbeitung und statistische Auswertung ................................................... 33 
4.1 Deskriptive Statistik 34

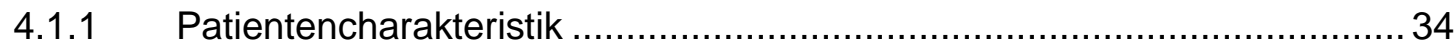

4.1.2 Ergebnisse der zahnärztlichen Untersuchung.................................... 36

4.1.3 Kiefergelenksdiagnostik bei Kindern mit juveniler Arthritis .......................38

4.2 Untersuchungen zum Zusammenhang zwischen oraler Gesundheit und Kiefergelenksbefunden.

4.3 Orale Gesundheit und Kiefergelenksbefunde in Abhängigkeit von Erkrankungsform, Erkrankungsdauer und Blutparametern

4.3.1 Vergleich von Parametern der oralen Gesundheit und röntgenologischer Kiefergelenksbefunde in Abhängigkeit von der Arthritisform.

4.3.2 Vergleich von Parametern der oralen Gesundheit in Abhängigkeit von Funktionseinschränkungen bei Mitbeteiligung oberer Gelenke.

4.3.3 Vergleich von Parametern der oralen Gesundheit und röntgenologischer Auffälligkeiten in Abhängigkeit von Blutparametern

4.3.4 Parameter der oralen Gesundheit und röntgenologische Auffälligkeiten in Abhängigkeit von der Erkrankungsdauer.

4.4 Untersuchungen zum Zusammenhang verschiedener Parameter der Kiefergelenksdiagnostik

4.4.1 Zusammenhang zwischen anamnestischer und klinischer

Kiefergelenksdiagnostik 54

4.4.2 Zusammenhang zwischen Röntgenscore und Anamnese .......................55

4.4.3 Zusammenhang zwischen Röntgenscore und klinischer Diagnostik ..........56

4.4.4 Zusammenhang zwischen Röntgenscore und einer Kombination aus Anamnese und klinischer Diagnostik. 58

4.5 Zusammenfassung der wichtigsten Ergebnisse. 61

5 Diskussion 62

5.1 Stärken und Limitationen 74

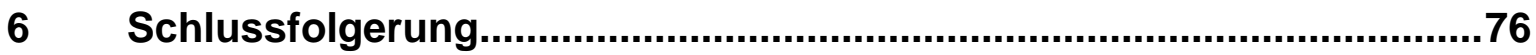

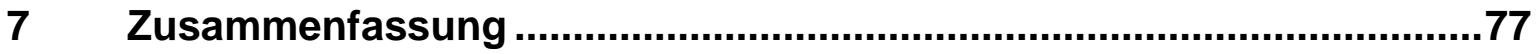

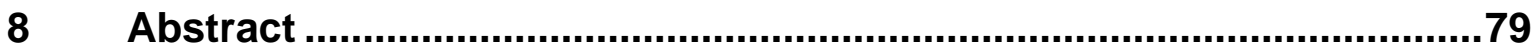




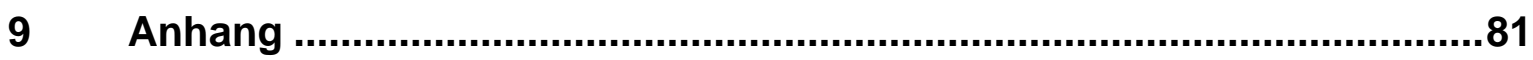

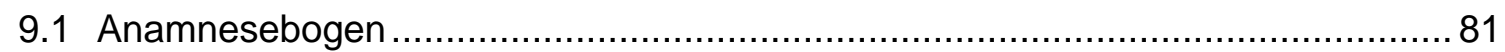

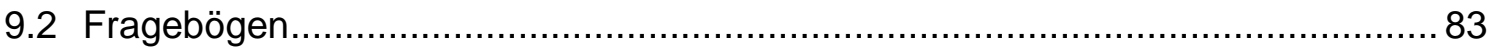

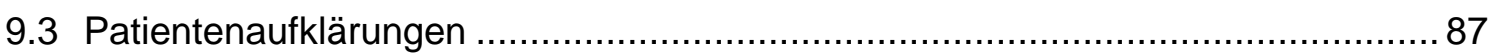

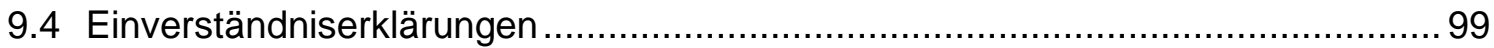

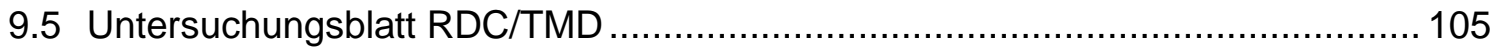

10 Literaturverzeichnis ..................................................................108 


\section{Abbildungsverzeichnis}

Abb. 1: Modell zur Entstehung einer Karies …………..................................

Abb. 2: Risikofaktoren für die Entstehung und Progression einer Parodontitis .......8

Abb. 3: Modulierende Faktoren des Krankheitsgeschehens ...............................13

Abb. 4: Darstellung genetischer Faktoren und entzündlicher Assoziationen zwischen Parodontitis und rheumatoider Arthritis ...................................22

Abb. 5: Definition der vier röntgenologischen Grade .........................................32

Abb. 6: Verteilung der PBI-Werte aller Messstellen ……...................................37

Abb. 7: Graphische Darstellung der Verteilung der PSI-Werte in den einzelnen

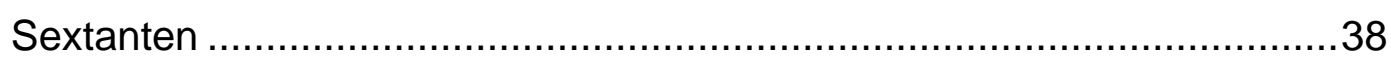

Abb. 8: Röntgenscore aller Kiefergelenke links- und rechtsseitig........................39

Abb. 9: Verteilung des maximal gemessenen PSI-Scores (PSI-Maximum) in Abhängigkeit von der Gelenkmitbeteiligung 49

Abb. 10: Verteilung des maximal gemessenen PSI-Scores (PSI-Maximum) in Abhängigkeit von den Blutparametern.

Abb. 11: Verteilung des Röntgenscores (RS) in Abhängigkeit von den

Blutparametern.

Abb. 12: Streudiagramm der Variablen Erkrankungsdauer und Kariesindex dmf-t/DMF-T .52

Abb. 13: Streudiagramm der Variablen Erkrankungsdauer und Kariesindex d-t/D-T .52

Abb. 14: Streudiagramm der Variablen Erkrankungsdauer und PBI-Maximum ....53

Abb. 15: Klinische Diagnostikparameter in Abhängigkeit von den

Anamneseparametern. .55

Abb. 16: Verteilung des Röntgenscores (RS) in Abhängigkeit von einzelnen

Anamneseparametern. .56

Abb. 17: Verteilung des Röntgenscores (RS) in Abhängigkeit von einzelnen klinischen Diagnostikparametern 58

Abb. 18: Verteilung des Röntgenscores in Abhängigkeit von der Diagnostik .59

Abb. 19: Verteilung des Röntgenscores in Abhängigkeit von einzelnen

Diagnostikparametern 60 


\section{Tabellenverzeichnis}

Tab. 1: Definition der JIA-Subgruppen nach Minden (2009) ...............................10

Tab. 2: Assoziationen von Parodontitis und rheumatoider Arthritis .......................21

Tab. 3: Übersicht über die fünf Grade des PBI...............................................27

Tab. 4: Übersicht über die parodontalen Befunde des PSI ................................27

Tab. 5: Patientencharakteristika Alter und Erkrankungsdauer nach Geschlecht...34

Tab. 6: Eingenommene Medikamente der Studienteilnehmer ..............................35

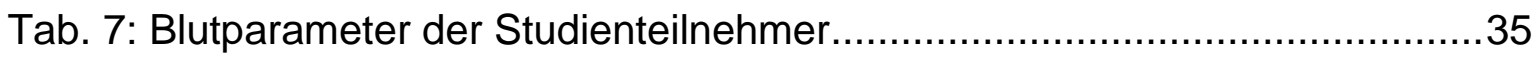

Tab. 8: Erkrankungsart und mitbeteiligte Gelenke ...........................................36

Tab. 9: Mittelwerte und Standardabweichungen der Karieserfahrung sowie des

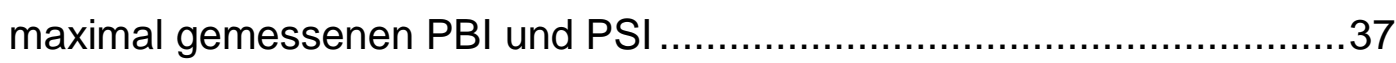

Tab. 10: Anamnestische, klinische und röntgenologische Parameter der Kiefergelenksdiagnostik

Tab. 11: Unterschiede bzgl. oraler Erkrankungen bei JIA-Patienten mit und ohne eingeschränkte MÖ

Tab. 12: Kreuztabelle zum Zusammenhang der klinisch diagnostizierten MÖ mit dem PSI-Maximum

Tab. 13: Kreuztabelle zum Zusammenhang der anamnestisch diagnostizierten MÖ mit dem PSI-Maximum.

Tab. 14: Unterschiede bzgl. oraler Erkrankungen bei JIA-Patienten mit und ohne Schmerzen.

Tab. 15: Kreuztabelle zum Zusammenhang klinisch diagnostizierter Schmerzen mit dem PSI-Maximum.

Tab. 16: Kreuztabelle zum Zusammenhang anamnestisch diagnostizierter Schmerzen mit dem PSI-Maximum

Tab. 17: Unterschiede bzgl. oraler Erkrankungen bei JIA-Patienten mit und ohne röntgenologischen Auffälligkeiten

Tab. 18: Kreuztabelle zum Zusammenhang röntgenologischer Kiefergelenksauffälligkeiten mit dem PSI-Maximum

Tab. 19: Tabellarische Darstellung der Unterschiede im Vorkommen oraler Erkrankungen bei Oligo- bzw. Polyarthritis 45

Tab. 20: Kreuztabelle zur Darstellung des Zusammenhangs zwischen dem PSIMaximum und der Oligo- bzw. Polyarthritis. 
Tab. 21: Kreuztabelle zur Darstellung des Zusammenhangs zwischen dem Röntgenscore und der Oligo- bzw. Polyarthritis 46

Tab. 22: Unterschiede bzgl. oraler Erkrankungen bei JIA-Patienten mit und ohne Erkrankung der oberen Extremitäten 47

Tab. 23: Kreuztabelle zum Zusammenhang der Mitbeteiligung oberer Gelenke und dem PSI-Maximum 48

Tab. 24: Statistische Auswertung des Zusammenhangs zwischen Blutparametern und dem oralen Entzündungsindex $\mathrm{PBI}$ 50

Tab. 25: Mittelwertunterschiede der Erkrankungsdauer in Abhängigkeit vom PSIMaximum und vom max. Röntgenscore .53

Tab. 26: Zusammenhang zwischen Anamnese und klinischer Diagnostik .54

Tab. 27: Kreuztabelle zum Zusammenhang zwischen Anamnese und Röntgenscore .55

Tab. 28: Kreuztabelle zum Zusammenhang zwischen Diagnostik und Röntgenscore .57 


\section{Abkürzungsverzeichnis}

ANA

Anti-ds-DNA

BOP

BS

$\mathrm{Cl}$

CPITN

CRP

CS

DMARD

DMF-S

DMF-T

HLA

$\lg$

IL

ILAR

INF

IS

JIA

k. A.

MAS

$\mathrm{MH}$

$\mathrm{MHC}$

MKG

MMP

MÖ

MRT

MTX

MW

NSAR

$\mathrm{OA}$

OPG antinukleärer Autoantikörper

Anti-doppelsträngige-DNS

Bleeding on Probing

Basistherapeutika

Cramers Index, Cramers V

Community Periodontal Index of Treatment Needs

C-reaktives Protein

Cortisonpräparate

Disease-Modifying Antirheumatic Drug

Decayed/Missing/Filled-Surfaces

Decayed/Missing/Filled-Teeth

Human leukocyte antigen

Immunglobulin

Interleukin

International League of Association for Rheumatology

Interferon

Immunsuppressiva

juvenile idiopathische Arthritis

keine Angabe

Makrophagenaktivierungssyndrom

Mundhygiene

Main histocompatibility complex

Mund-Kiefer-Gesicht

Matrixmetalloproteinasen

Mundöffnung

Magnetresonanztomographie

Methotrexat

Mittelwert

nichtsteroidale Antirheumatika

Oligoarthritis

Orthopantomogramm 
PA Polyarthritis

PBI Papillen-Blutungs-Index

PSI Parodontaler Screening-Index

RA rheumatoide Arthritis

RDC/TMD Research Diagnostic Criteria for Temporomandibular Disorder

$\mathrm{RF}$

Rheumafaktor

RS

Röntgenscore

SA

Standardabweichung

SPSS

Statistical Package for the Social Sciences

TMJ

Temporomandibular joint

TNF

Tumornekrosefaktor

WHO

World Health Organisation 


\section{Einleitung}

Der Begriff „juvenile idiopathische Arthritis“ (JIA, ältere Synonyme: juvenile rheumatoide Arthritis, juvenile chronische Arthritis) beschreibt eine Gruppe chronisch entzündlicher Gelenkerkrankungen, welche zusammenfassend in der pädiatrischen Rheumatologie die häufigsten rheumatischen Erkrankungsformen darstellen (Consolaro et al. 2012). Die JIA ist demnach keine spezielle Erkrankung, sondern vielmehr die Diagnose jeder kindlichen Arthritis ohne erkennbare Ursache. Die Ätiologie bleibt bis heute unklar, wobei zahlreiche Faktoren wie Umwelt- oder genetische Einflüsse für die Erkrankung verantwortlich gemacht werden (Huang 2012).

Bei der interdisziplinären Zusammenarbeit von Ärzten und Zahnärzten gewinnen Zusammenhänge zwischen Allgemeinerkrankungen, wie der JIA, und der oralen Gesundheit zunehmend an Bedeutung. Ein gehäuftes Vorkommen oraler Erkrankungen in dieser Patientengruppe, wie z. B. der Karies, wurde bereits von einigen Autorengruppen beschrieben (Bhatt et al. 2014, Barr et al. 2008, Welbury et al. 2003, Walton et al. 2002, Walton et al. 2000, Tanchyk 1991, Siamopoulou et al. 1989, Storhaug und Holst 1987). Die Gründe für ein vermehrtes Auftreten oraler Erkrankungen sind zahlreich. Allerdings weisen nicht alle JIA-Patienten orale Defizite auf. Die Erkennung von begünstigenden Risikofaktoren ist somit von großer Bedeutung (Walton et al. 2000).

Einige Autoren beschreiben eine erhöhte Kariesanfälligkeit durch zuckerhaltige Medikamente (Shaw und Glenwright 1989), veränderte Ernährungsweisen (Tanchyk 1991) oder eine veränderte Speichelzusammensetzung (Siamopoulou et al. 1989). Ein Weiterer bedeutender Grund für eine erhöhte Kariesaktivität könnten Funktionseinschränkungen sein, unter welchen JIA-Patienten leiden und die eine adäquate Mundhygiene erschweren (Walton et al. 2000). Hier wäre insbesondere die eingeschränkte Mundöffnung (MÖ) bei vorliegendem Kiefergelenksbefall sowie eine feinmotorische Einschränkung bei Mitbeteiligung oberer Extremitäten zu nennen (Walton et al. 2000).

Ob bei jungen Rheumapatienten vermehrt Krankheitsanzeichen einer Gingivitis oder Parodontitis (PD) auftreten und somit Zusammenhänge zwischen der JIA und parodontalen Erkrankungen bestehen, wird kontrovers diskutiert (Ahmed et al. 2004, Miranda et al. 2003, Siamopoulou et al. 1989). Der Zusammenhang zwi- 
schen der PD und der rheumatoiden Arthritis wurde bei Erwachsenen weit besser untersucht als bei Kindern. Hier können mittlerweile zahlreiche Begründungen genannt werden, warum eine gesicherte Assoziation zu existieren scheint (Calderaro et al. 2017, Holmstrup et al. 2017, Tang et al. 2017, Kaur et al. 2013, Ortiz et al. 2009). Ähnlich der rheumatoiden Arthritis stellen fortgeschrittene parodontale Erkrankungen mit Attachmentverlust (Parodontitis) destruktive Entzündungsgeschehen zwischen knöchernen Strukturen und umgebenden Gewebe dar (Detert et al. 2010). Immunologische Prozesse, das Vorkommen parodontitisassoziierter Mikroorganismen in der Synovialflüssigkeit oder gleichartige genetischen Prädispositionen sind mögliche Erklärungen für die Assoziationen der PD mit der RA (Białowąs et al. 2014, El-Shinnawi und Soory 2013, Ogrendik 2013, Engelmann und MüllerHilke 2009). Hauptrisikofaktor für parodontale Erkrankungen ist die Biofilmakkumulation auf der Zahn- bzw. Wurzeloberfläche (Schroeder 1997). Somit könnten die für die erhöhte Kariesaktivität beschrieben Risikofaktoren, wie eine eingeschränkte Mundhygiene, auch für das Entstehen einer Gingivitis oder Parodontitis ursächlich sein. Ebenfalls zu berücksichtigen ist die Einnahme von Medikamenten, welche zum Teil orale Nebenwirkungen wie Stomatitis, Ulzerationen und Gingivawucherungen hervorrufen können (Barr et al. 2008). Über ein vermehrtes Auftreten von Gingivitis bei JIA-Patienten wurde in einigen Studien berichtet (Ahmed et al. 2004, Savioli 2004, Welbury et al. 2003). Zusammenhänge der JIA mit der Parodontitis wurden bisher allerdings selten beobachtet (Hucke 2014).

Es wird beschrieben, dass es bei der JIA in bis zu 87\% der Fälle zu einer Mitbeteiligung der Kiefergelenke kommt (Tzaribachev et al. 2010). Bei fast der Hälfte aller betroffenen Kinder verläuft die Kiefergelenkentzündung asymptomatisch (Twilt et al. 2008). Allerdings ist eine frühe Erkennung und Behandlung der Entzündung für das regelrechte Wachstum und der Vermeidung von Spätfolgen von großer Bedeutung. Auch nicht auf die JIA spezialisierte Zahnärzte sollten mit zur Verfügung stehenden Mitteln eine Kiefergelenkerkrankung und deren Schweregrad möglichst sicher erkennen zu können. Verfügbare Mittel sind hierbei anamnestische und klinische Diagnoseparameter sowie als bildgebende Diagnostik in den meisten Fällen das Orthopantomogramm (OPG). Eine gesicherte Diagnostik konnte mit oben genannten Maßnahmen bisher aber nicht gewährleistet werden (Keller et al. 2015, Abramowicz et al. 2013, Koos et al. 2011, Argyropoulou et al. 2009, Weiss et al. 2008, Billiau et al. 2007, Twilt et al. 2004). 
Ziel dieser Studie war es, den Mundgesundheitszustand und die Kiefergelenke bzw. die Kiefergelenksdiagnostik bei Kindern und Jugendlichen mit der Diagnose "JIA“ zu untersuchen. Dabei sollten Zusammenhänge zu Patienten- und Erkrankungscharakteristika berücksichtigt werden, um mögliche Einflussfaktoren auf die Mundgesundheit und bestehende Kiefergelenksbefunde bei diesem Patientenklientel zu ermitteln.

Des Weiteren sollten Zusammenhänge zwischen anamnestischen, klinisch funktionellen und röntgenologischen Kiefergelenksbefunden festgestellt werden.

In Berücksichtigung der bisherigen Erkenntnisse zum Forschungsgegenstand und der Zielstellung der Untersuchung wurden die nachfolgenden Arbeitshypothesen gebildet:

1. JIA-Patienten weisen einen dentalen und parodontalen Behandlungsbedarf auf. Es sind Unterschiede bei der oralen Gesundheit in Abhängigkeit der Erkrankungsform und Erkrankungsdauer, der Mitbeteiligung oberer Extremitäten, vorliegender Blutparameter und auffälligen Kiefergelenksbefunden zu beobachten.

2. JIA-Patienten zeigen anamnestische, klinisch funktionelle und röntgenologische Auffälligkeiten in der Kiefergelenksdiagnostik. In Abhängigkeit der Erkrankungsform, Erkrankungsdauer und Blutparameter sind strukturelle Unterschiede der Kondylen im OPG erkennbar. In Bezug auf die Röntgenauswertung bestehen Zusammenhänge zur oralen Gesundheit und zu auffälligen Parametern der anamnestischen und klinischen Kiefergelenksdiagnostik. 


\section{Literaturübersicht}

\subsection{Karies}

\subsubsection{Definition und Epidemiologie}

Bei der Karies handelt es sich um einen lokalisierten, pathologischen Vorgang bakteriellen Ursprungs, bei dem es zu einem fortschreitenden Verfall (Demineralisation) der Zahnhartsubstanz kommt. Die kariöse Erkrankung ist durch Phasen der Stagnation, Remission und Progression gekennzeichnet (Hellwig et al. 2009). Die fünfte Deutsche Mundgesundheitsstudie (DMS V) aus dem Jahre 2016 hat gezeigt, dass Kinder mit 12 Jahren durchschnittlich eine Karieserfahrung (DMF-T) von 0,5 aufwiesen. $81,3 \%$ der Kinder (12 Jahre) hatten ein kariesfreies Gebiss (Jordan und Micheelis 2016). Die Zahl der kariesfreien Gebisse hat sich damit in den Jahren 1997 bis 2014 praktisch verdoppelt. Eine mögliche Erklärung für diesen Kariesrückgang wird in regelmäßigen zahnärztlichen Kontrolluntersuchungen gesehen (Jordan und Micheelis 2016). Trotz einem anhaltenden Kariesrückgang im bleibenden Gebiss scheint die Prävalenz frühkindlicher Karies auf stabilem Niveau zu verbleiben und liegt gemäß verschiedenen regionalen Studien in Deutschland zwischen $10 \%$ und $15 \%$ (Jordan et al. 2016). Die frühkindliche Karies gilt auch heute noch als eine der häufigsten Erkrankungsformen bei Kleinkindern (Jordan et al. 2016).

\subsection{2 Ätiologie und Pathogenese}

Karies wird als eine multifaktoriell bedingte Erkrankung angesehen. Die bereits 1889 von Miller beschriebene chemoparasitäre Kariesentstehungstheorie hat heute noch weitestgehend ihre Gültigkeit (Miller 1889). Das Vorhandensein potenziell kariogener Mikroorganismen ist hierbei von entscheidender Bedeutung. Eine herausragende Rolle spielt insbesondere das Bakterium Streptococcus mutans. Dieses Bakterium besitzt die Fähigkeit unter Säurebildung Monosaccharide, wie Glucose und Fructose, abzubauen (Hellwig et al. 2009). Dies führt im Schmelz zu interprismatischen Auflösungserscheinungen. Phosphat- und Hydroxylionen werden aus dem Kristallgitter herausgelöst, was den kariösen Demineralisationsprozess einleitet (Hellwig et al. 2009, Klimm 1997). Keyes (1962) erweiterte die chemoparasitäre Theorie und beschreibt ein Zusammenspiel von kariogenen Mikroorga- 
nismen, Substrat und dem Wirt. Für den Stoffwechsel kariogener Mikroorganismen gelten Substrate, wie Glukose und Fruktose und insbesondere das Disaccharid Saccharose, als wesentliche Grundvoraussetzung (Newbrun 1969, Neff 1967). Individuelle, wirtsbedingte Faktoren wirken sich auf die Entstehung und das Fortschreiten einer Karies aus. Hierzu zählen u. a. der Speichel, die Zahnform, die Schmelzreifung und das Fluoridangebot (Klimm 1997). Die durch Keyes (1962) modifizierte Kariestheorie wurde nachfolgend nochmals um den Faktor Zeit erweitert, da eine Karies nur entsteht, wenn sich über einen Zeitraum ein strukturierter Biofilm (Plaque) auf der Zahnoberfläche etabliert hat und es zu häufigen bzw. lang anhaltenden Säureangriffen kommt (Hellwig et al. 2009, Konig 1985, Gustafsson et al. 1954). Hierbei stellt der Biofilm einen zähen und verfilzten Zahnbelag aus Speichelbestandteilen, bakteriellen Stoffwechselprodukten, Nahrungsresten und Bakterienzellen dar (Hellwig et al. 2009). Abbildung 1 zeigt eine Übersicht direkter und indirekter Einflussfaktoren auf die Entstehung einer Karies.

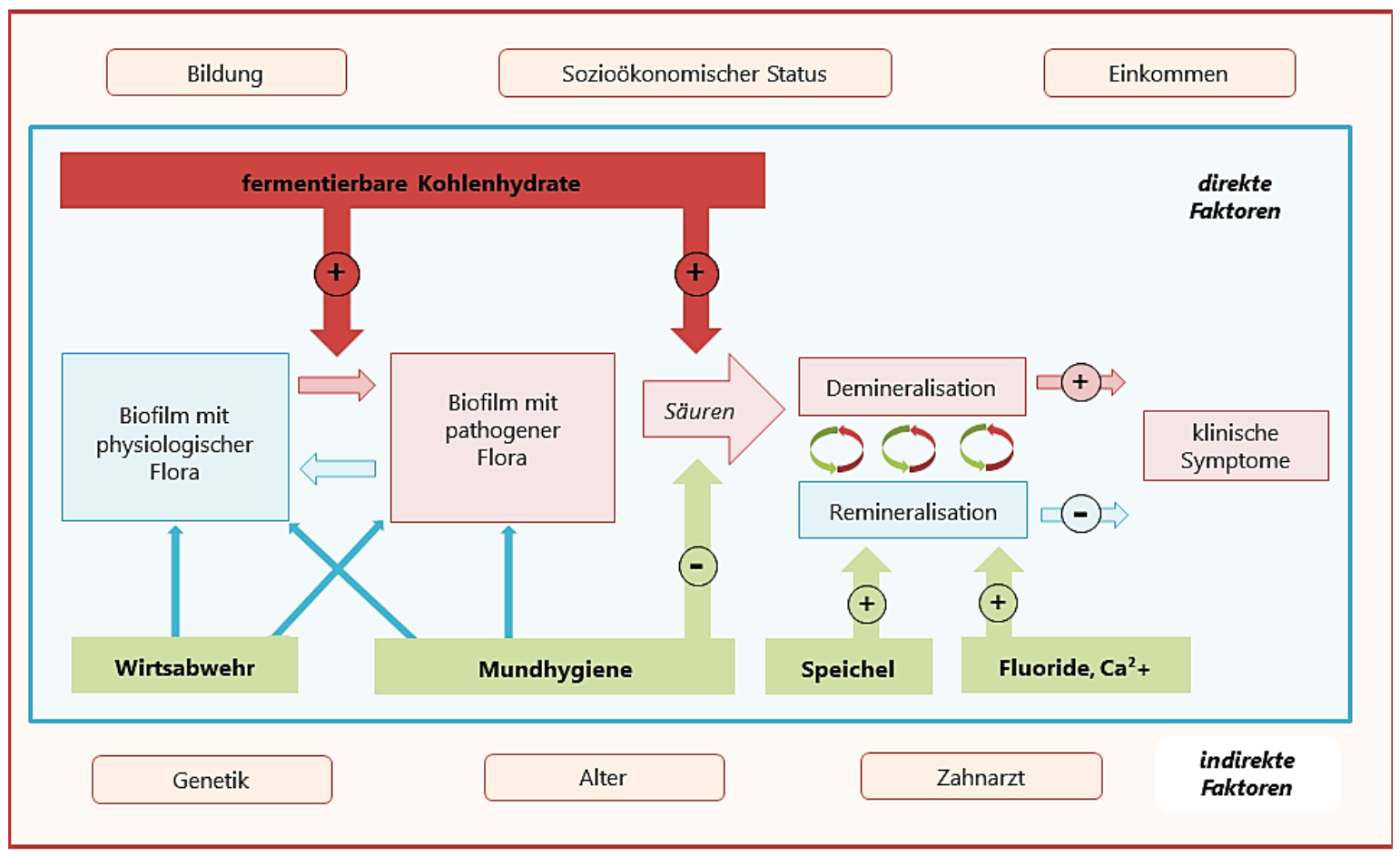

Abb. 1: Modell zur Entstehung einer Karies, eigene Darstellung modifiziert nach Paris und Meyer-Lückel (2012, S.73)

Heute wird die Kariesentstehung unter ökologischen Gesichtspunkten betrachtet (Lehmann 1991). Marsh und Martin führten 1992 den Begriff der ökologischen Plaquehypothese ein (Marsh 2003). Diese Theorie beschreibt bei neutralem pHWert nur einen sehr geringen Anteil kariogener Bakterien. De- und Remineralisati- 
onsvorgänge befinden sich im Gleichgewicht. Erst die häufige Zufuhr niedermolekularer Kohlenhydrate kann zu einer Senkung des pH-Wertes unter einen kritischen Punkt führen. Folgend kommt es zu einer Vermehrung kariogener Mikroorganismen und das Gleichgewicht zwischen De- und Remineralisation verschiebt sich in Richtung Demineralisation (Marsh 2003).

\subsection{Parodontale Erkrankungen}

\subsubsection{Definition und Epidemiologie}

Parodontale Erkrankungen stellen entzündliche Vorgänge der zahnumgebenden Gewebe dar. Die Gingivitis und die Parodontitis sind die am häufigsten auftretenden Erkrankungsformen (Peter et al. 2014).

Die Gingivitis ist eine reversible, primär bakteriell verursachte Entzündung des marginalen Parodonts (Lyons et al. 1959). Sie kann als eine lokale, opportunistische Infektion bezeichnet werden und ist charakterisiert durch eine Schwellung und Rötung der Gingiva bei erhöhter Blutungsneigung (Kandilakis und Lang 2003, Page und Schroeder 1976). Lokale und systemische Faktoren erleichtern entweder die Plaqueentwicklung oder erhöhen die Empfindlichkeit des Wirts gegenüber bakteriellen Angriffen (Kandilakis und Lang 2003). Die Plaque-induzierte Gingivitis kann in vier Untergruppen eingeteilt werden (Armitage 1999):

- Gingivitis, ausschließlich plaqueinduziert

- Gingivitis, modifiziert durch systemische Faktoren

- Gingivitis, modifiziert durch Medikamente

- Gingivitis infolge Mangelernährung

Von der Plaque-induzierten Gingivitis wird die nicht-plaque-induzierte Gingivitis unterschieden (Armitage 1999). Die fünfte deutsche Mundgesundheitsstudie (DMS V) offenbarte, dass in Deutschland ca. ein Fünftel (22,3\%) der 12-jährigen Kinder anhand des Papillen-Blutungs-Index (PBI) entzündungsfreie Gebisse aufwiesen. Bei einem Großteil der kindlichen Population (68,5\%) wurden geringe Entzündungszeichen (Grad 1 und 2) beobachtet. Ausgeprägte Entzündungen (Grad 3 und 4) traten mit einer Prävalenz unter 10\% auf, wobei der Grad 4 mit 0,1\% sehr selten vorkam (Jordan und Micheelis 2016). 
Die Parodontitis (PD) ist eine bakteriell bedingte Entzündung des gesamten Zahnhalteapparates. Sie gilt als opportunistische, multifaktoriell bedingte Erkrankung. Im Gegensatz zur Gingivitis stellt die PD eine irreversible Erkrankungsform dar und kann in fortgeschrittenen Erkrankungsstadien zu ausgeprägten Knochendestruktionen und Zahnverlust führen (Lindroth und Park 2013). Die bedeutendsten Komponenten der PD werden nach Schroeder (1997) in dem Abbau des Alveolarknochens, der Tiefenproliferation und Ulzeration des Saumepithels sowie in einem raschen bindegewebigen Attachementverlust gesehen. In der Klassifizierung der Parodontalerkrankungen wird die PD nochmals in sich unterscheidende Krankheitsbilder unterteilt (Armitage 1999).

- Chronische Parodontitis

- Aggressive Parodontitis

- Parodontitis als Manifestation einer Systemerkrankung

- Nekrotisierende Parodontalerkrankungen

- Parodontalabszesse

- Parodontitis im Zusammenhang mit endodontalen Läsionen

- Entwicklungsbedingte oder erworbene Deformationen und Zustände

Aktuelle Daten zur Epidemiologie der Parodontitis bei Kindern und Jugendlichen liegen nicht vor. Auch die DMS V macht diesbezüglich keine Angaben.

\subsection{2 Ätiologie und Pathogenese}

Die Plaque-induzierte Gingivitis ist eine entzündliche Wirtsantwort auf den bakteriellen Reiz. Sie kann sich unter verschiedenen Umständen in eine Parodontitis entwickeln (Güntsch et al. 2011). Wie oben bereits aufgeführt, gilt die Parodontitis als opportunistische, multifaktoriell bedingte Erkrankung, deren Ausbruch und Schweregrad durch eine komplexe Interaktion von zahlreichen umweltbedingten, erworbenen oder genetischen Risikofaktoren bedingt ist. In Abbildung 2 sind die Risikofaktoren für die Entstehung und Progression der PD dargestellt. 


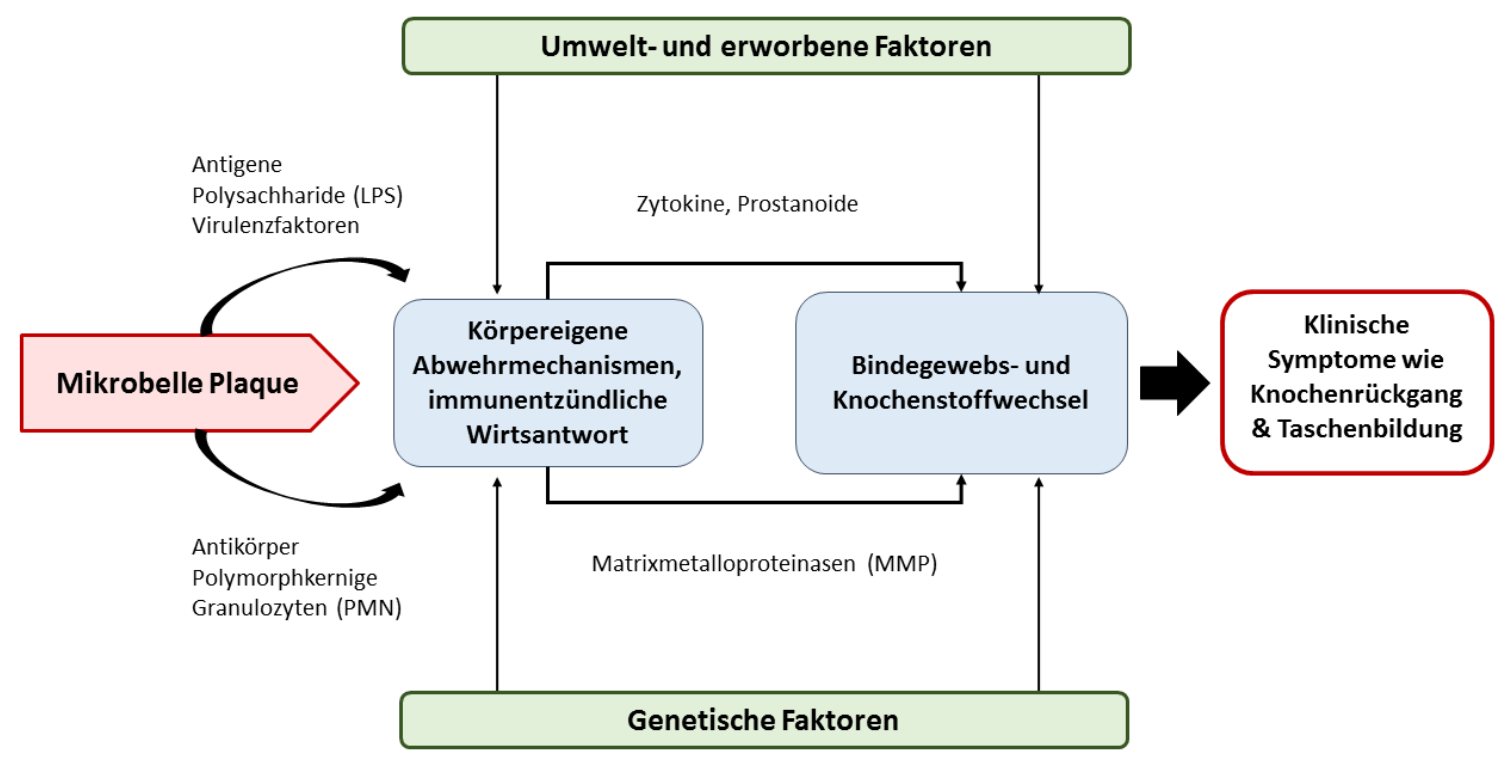

Abb. 2: Risikofaktoren für die Entstehung und Progression einer Parodontitis, modifiziert nach Page und Kornman (1997, S.11)

Als primärer Risikofaktor gilt die Etablierung eines bakteriellen Biofilms (Schroeder 1997). Im Gegensatz zur planktonischen Lebensweise ist durch die Kolonisation von Bakterien in einem Biofilm die Virulenz im Vergleich zu Einzelbakterien verstärkt und die Überlebensfähigkeit gesteigert (Güntsch et al. 2011). Im Laufe des Dickenwachstums des Biofilms verschieben sich die Anteile der Bakterien. Es entsteht zunehmend ein anaerobes Milieu, sodass eine Kolonisation fakultativ und obligat anaerober, gramnegativer Bakterien ermöglicht wird (Mengel und Floresde-Jacoby 2000). Durch die Vertiefung des Sulcus gingivae und die ödematöse Schwellung der Gingiva (Gingivitis), als entzündliche Reaktion auf den supragingivalen Biofilm, entsteht frühzeitig ein subgingivaler Bereich (Müller 2012). Parodontalen Erkrankungen sind Infektionen, die im Zusammenhang mit spezifischen, potenziell pathogenen Bakterien stehen, welche in dem subgingivalen Bereich kolonisieren (Wolf et al. 2012, Socransky et al. 1998).

Virulenzfaktoren der pathogenen Bakterien, wie Toxine, Enzyme und metabolische Produkte, tragen zu der direkten parodontalen Destruktion bei. Die Pathogenität der Bakterien kommt teilweise erst durch ihr Eindringen in das Gewebe voll zum Tragen (Listgarten 1987). Enzyme, wie z. B. Proteinasen, werden freigesetzt, um nachfolgend Matrixmetalloproteine zu aktivieren. Immunglobuline und Faktoren des Komplementsystems werden abgebaut, und die Antigen-AntikörperAbwehr wird inhibiert. Neben Enzymen und Stoffwechselprodukten können auch antigene Toxine, wie beispielsweise Lipopolysaccharide, das Gewebe schädigen 
(Stein et al. 1999). Allerdings manifestiert sich eine parodontale Erkrankung nicht universell bei allen Patienten mit schlechter Mundhygiene und Beherbergung parodontaler Mikroorganismen. Eine Progression erfordert auch einen anfälligen Wirt. Demnach ist die Pathogenese und Ausprägung parodontaler Erkrankungen neben dem Vorliegen von Mikroorganismen von vielen weiteren Risikofaktoren, wie systemischen Erkrankungen, genetischen Faktoren, Umweltfaktoren, Alter, Geschlecht und Mundhygieneverhalten abhängig (Lindroth und Park 2013, Van der Velden et al. 2006, Van Dyke et al. 2005).

Zu den Umwelt- und erworbenen Risikofaktoren zählen u. a. das Rauchen, psychischer Stress und Depression, Alkohol, Fettleibigkeit und Allgemeinerkrankungen, wie das Vorliegen einer HIV-Infektion, Diabetes mellitus, Blutkrankheiten und Osteoporose (Reynolds 2014, Wolf et al. 2012, Kinane et al. 2006). Auch bestimmte Ernährungsweisen und die Einnahme von Medikamenten, insbesondere Immunsuppressiva, gelten als Einflussfaktoren in der Ätiologie und Pathogenese entzündlicher Parodontopathien (Raindi 2016, Barr et al. 2008). Endogene Faktoren wie Speichelmangel oder -zusammensetzung, Mundatmung, Zahnfehlstellungen und exogene mechanische oder chemische Reize gelten als lokale Kofaktoren, welche das Krankheitsgeschehen modifizieren (Reynolds 2014, Wolf et al. 2012, Kinane et al. 2006). Genetischen Risikofaktoren bzw. chromosomale Anomalien können sogar allein für das Auslösen der parodontalen Erkrankung verantwortlich sein. Meist handelt es sich jedoch um genetische Kofaktoren in einem multifaktoriellen Krankheitsgeschehen (Wolf et al. 2012).

\subsection{Juvenile idiopathische Arthritis}

\subsubsection{Definition und Epidemiologie}

Für die chronische Arthritis beim Kind wurde unter der Zusammenarbeit der ILAR (International League of Association for Rheumatology), der WHO (World Health Organisation) und Kinderrheumatologen im Jahre 1995 die Bezeichnung juvenile idiopathische Arthritis (JIA) eingeführt (Carrasco 2015, Huang 2012, Hofer und Southwood 2002). Die JIA ist die häufigste rheumatische Erkrankung bei Kindern und Jugendlichen (Carrasco 2015, Manners und Bower 2002). Allerdings handelt es sich nicht um eine einzelne Erkrankung, sondern um eine heterogene Gruppe aller Formen der chronischen Arthritiden, die vor dem 16. Lebensjahr beginnen, 
länger als 6 Monate andauern und keine erkennbare Ursache haben (Ravelli und Martini 2007). Nach der aktuellen ILAR-Klassifikation wird die JIA in sieben Subgruppen aufgeteilt (Minden 2009) (Tab. 1).

Tab. 1: Definition der JIA-Subgruppen nach Minden (2009)

\begin{tabular}{|c|c|}
\hline Subgruppe & Definition \\
\hline Oligoarthritis & $\begin{array}{l}\text { Entzündung von maximal } 4 \text { Gelenken während der ersten } 6 \text { Er- } \\
\text { krankungsmonate }\end{array}$ \\
\hline - persistierende Form & $\begin{array}{l}\text { nach den ersten } 6 \text { Erkrankungsmonaten werden nicht mehr als } 4 \\
\text { Gelenke in den Entzündungsprozess einbezogen }\end{array}$ \\
\hline - erweiterte Form & $\begin{array}{l}\text { nach den ersten } 6 \text { Erkrankungsmonaten werden } 5 \text { und mehr Ge- } \\
\text { lenke in den Entzündungsprozess einbezogen }\end{array}$ \\
\hline $\begin{array}{l}\text { seronegative } \\
\text { Polyarthritis }\end{array}$ & $\begin{array}{l}\text { Entzündung von mehr als } 4 \text { Gelenken während der ersten } 6 \text { Er- } \\
\text { krankungsmonate, kein Nachweis von Rheumafaktoren }\end{array}$ \\
\hline seropositive Polyarthritis & $\begin{array}{l}\text { Entzündung von mehr als } 4 \text { Gelenken während der ersten } 6 \text { Er- } \\
\text { krankungsmonate, Nachweis von Rheumafaktoren }\end{array}$ \\
\hline Psoriasisarthritis & $\begin{array}{l}\text { Arthritis und Schuppenflechte oder Arthritis und Wurstfinger/- } \\
\text { zehe, Nagelveränderungen und/oder ärztlicherseits bestätigte } \\
\text { Schuppenflechte bei einem Verwandten 1. Grades }\end{array}$ \\
\hline $\begin{array}{l}\text { enthesitisassoziierte } \\
\text { Arthritis }\end{array}$ & $\begin{array}{l}\text { Krankheitsbild mit Arthritis und Sehnenansatzent-zündungen } \\
\text { (Enthesitis), das zu den Spondylarthropathien gehört und in eine } \\
\text { ankylosierende Spondylitis münden kann }\end{array}$ \\
\hline systemische Arthritis & $\begin{array}{l}\text { neben der Arthritis durch Fieber, Hauterscheinungen, Lymphkno- } \\
\text { tenvergrößerungen, Leber- und/oder Milz-vergrößerung und/oder } \\
\text { Entzündungen des Herzbeutels, des Brust- oder Bauchfells ge- } \\
\text { kennzeichnet }\end{array}$ \\
\hline weitere Arthritiden & $\begin{array}{l}\text { Arthritiden, die nicht eindeutig den definierten Subgruppen } 1 \text { bis } 6 \\
\text { zugeordnet werden können }\end{array}$ \\
\hline
\end{tabular}

Die Unterscheidung findet sowohl aufgrund von Geschlecht, Anzahl und Muster der betroffenen Gelenke als auch in Bezug auf eine Mitbeteiligung innerer Organe statt. Durch das Vorhandensein weiterer Erkrankungszeichen, wie z. B. Fieber, Exanthemen, Psoriasis, entsprechender Laborbefunde, wie Immunglobulin-MRheumafaktoren (IgM-Rheumafaktor) oder des genetischen Merkmals human leukocyte antigen B27 (HLA-B27), sind weitere Subgruppen definiert (Manger 2011). 
Die Diagnosefindung kann durch spezifische Marker unterstützt werden (Hucke 2014). Ein typischer Parameter ist hierbei das auf ein verstärktes Krankheitsgeschehen hinweisende C-reaktive Protein (CRP) (Dick 2000). Neben einigen weniger spezifischen Blutparametern, welche die Krankheitsaktivität widerspiegeln, können das Auftreten von Autoantikörper, wie der antinukleäre Antikörper (ANA) und Rheumafaktoren (RF), sowie das Histokompatibilitätsantigen HLA-B27 wichtige diagnostische Hinweise liefern (Dick 2000).

Bei der JIA handelt es sich um eine relativ seltene Erkrankung im Kindesalter, deren Häufigkeit für verschiedene Bevölkerungsgruppen sehr unterschiedlich angegeben wird. Es kann davon ausgegangen werden, dass weltweit zwischen 16 und 150/100.000 Kinder erkrankt sind. Die weltweite Neuerkrankungsrate beträgt 2 bis 20/100.000 Kinder (Kahn 2011). Für Deutschland wird eine Prävalenz von 2030/100.000 Kindern und eine Inzidenz von 5-6/100.000 angegeben (von Koskull et al. 2001). Wie auch bei anderen rheumatischen Erkrankungen sind Mädchen doppelt so häufig von der JIA betroffen wie Jungen (Kahn 2011).

\subsection{2 Ätiologie und Pathogenese}

Die genauen Ursachen der JIA sind bisher noch nicht geklärt. Man nimmt an, dass für den Ausbruch der Erkrankung ein gemeinsames Auftreten genetischer und umweltbedingter Faktoren verantwortlich ist (Oberle et al. 2014). Es ist davon auszugehen, dass es eine genetische Veranlagung gibt, an Rheuma zu erkranken. Eine familiäre Häufung der Erkrankung konnte entsprechend beobachtet werden. Außerdem sind genetische Merkmale bekannt, die bei Patienten mit bestimmten Rheumaformen gehäuft auftreten (Oberle et al. 2014). Vielfach wird hierbei eine HLA-Assoziation beschrieben (Oberle et al. 2014, Prahalad et al. 2012, Yanagimachi et al. 2011, Berntson et al. 2008, Thomson et al. 2002, Savolainen et al. 1998, Fernandez-Viña et al. 1994, Ploski et al. 1993). Diese Histokompatibilitätsantigene (MHC, main histocompatibility complex, beim Menschen auch HLA= human leukocyte antigens genannt) sind auf allen Geweben des Organismus exprimierte Glykoproteine, die vom Immunsystem eines anderen Organismus als fremd erkannt werden. Der MHC-Komplex wird von mehreren Genen codiert. Da es in der Bevölkerung verschiedene Allele für jedes dieser Gene gibt, resultiert eine große Variabilität. Assoziationen der JIA sowohl mit Genvarianten der HLAKlasse1 (HLA-A2, und HLA-B27) als auch mit Varianten der HLA-Klasse2 (HLA- 
DRB1 und HLA-DP) konnten nachgewiesen werden (Oberle et al. 2014, Huang 2012, Prakken et al. 2011).

Nicht HLA assoziierte Gene, die mit der JIA in Verbindung gebracht werden, sind für die Zytokinexpression sowie für weitere immunregulatorische Prozesse verantwortlich (Oberle et al. 2014, Prakken et al. 2011). Eine entscheidende Rolle im Krankheitsgeschehen der JIA spielt die überschießende Aktivierung des Immunsystems. Erhöhte Level proinflammatorischer Zytokine, wie Tumornekrosefaktoralpha (TNF-alpha), Interleukin-1 (IL-1), Interleukin-6 (IL-6) und Interferon-gamma (INF- $\gamma$ ), in der Synovialflüssigkeit und im Serum von JIA-Patienten deuten auf eine abnormale Expression zytokinproduzierender und zytokinregulierender Gene hin (Woo und Colbert 2009, Prahalad und Glass 2008). Erhöhte Genexpressionen im Zusammenhang mit dem Krankheitsgeschehen der JIA werden beispielsweise für den TNF-alpha-Polymorphismus, den IL-1-, IL-6- oder den IL-10Polymorphismus beschrieben (Omoyinmi et al. 2012, Scardapane et al. 2012, Prahalad et al. 2008, Stock et al. 2008). Als weitere Ursache gesteigerter Expressionen werden fehlerhafte DNA-Methylierungen genannt (Ellis et al. 2012a, Ellis et al. 2012b).

Für die Vermutung einer Autoimmunerkrankung sprechen nachgewiesene Immunphänomene wie Auto-Antikörper gegen IgG (Rheumafaktor), antinukleäre Antikörper (ANA) und Antikörper gegen citrullinierte Peptide (Omar et al. 2013, Tebo et al. 2012, Nisihara et al. 2009). Sie sind für das Vorliegen einer rheumatologischen Krankheit aber nicht absolut spezifisch und schließen bei fehlendem Nachweis eine entzündlich-rheumatologische Krankheit nicht aus (Kavanaugh et al. 2000).

Des Weiteren werden zahlreiche äußere Einflüsse diskutiert, welche am Ausbruch der JIA oder an einem verstärkten Krankheitsgeschehen beteiligt sind. Mikrobiologische Einflüsse, wie virale und bakterielle Infektionen kommen in Betracht (Oberle et al. 2014). Von besonderem Interesse sind hierbei Untersuchungen zu Assoziationen zwischen der Stillzeit und der Ausbildung der rheumatischen Erkrankung. Bezüglich der JIA berichten einige Autoren über eine JIA-protektive Wirkung bestimmter Muttermilchsubstanzen, welche dem Kind bei fehlender Stillzeit fehlen könnten (Young et al. 2007, Mason et al. 1995). Auch Traumata und seelische Belastungssituationen, wie physischer und psychischer Stress, werden als Triggerfaktoren genannt (Neufeld et al. 2013). Bei Erwachsenen gilt das Rauchen als 
bedeutender Risikofaktor für die Erkrankung an einer Arthritis, da es negative Einflüsse auf das Immunsystem ausübt, wie beispielsweise eine Reduktion natürlicher Killerzellen oder einer Beeinträchtigung von Lymphozytenfunktionen (Sugiyama et al. 2010). Studien, welche den Einfluss des Rauchens während der Schwangerschaft untersuchten, erbrachten jedoch sehr heterogene Ergebnisse (Carlens et al. 2009, Jaakkola und Gissler 2005). Generell sind äußere Einflüsse auf das Krankheitsgeschehen sehr schwierig zu beurteilen und die Datenlage nicht eindeutig. Definitionsgemäß beginnt die JIA somit meist idiopathisch, also ohne erkennbare äußere Ursache (Oberle et al. 2014).

\subsubsection{Medikation}

Für die medikamentöse Behandlung der JIA stehen hauptsächlich nichtsteroidale Antirheumatika (NSAR), intraartikuläre Glukokortikoidinjektionen und die Medikamentenklasse der Basistherapeutika (BS), auch DMARDs (Disease-Modifying Antirheumatic Drugs) genannt, zur Verfügung. Diese wirken sowohl auf das Immunsystem als auch auf die Entzündungsprozesse an sich (Abb. 3). Die in der JIATherapie ebenfalls verwendeten Immunsuppressiva (IS), wie das Cyclosporin A, können ebenfalls zu den Basistherapeutika gezählt werden (Heubner et al. 2002). In den letzten Jahren kamen zusätzlich die sog. Biologika, einschließlich TumorNekrose-Faktor-Inhibitoren, Interleukin-1-Inhibitoren und Interleukin-6-Inhibitoren mit vielversprechenden Behandlungserfolgen zunehmend zum Einsatz (Harris et al. 2013).

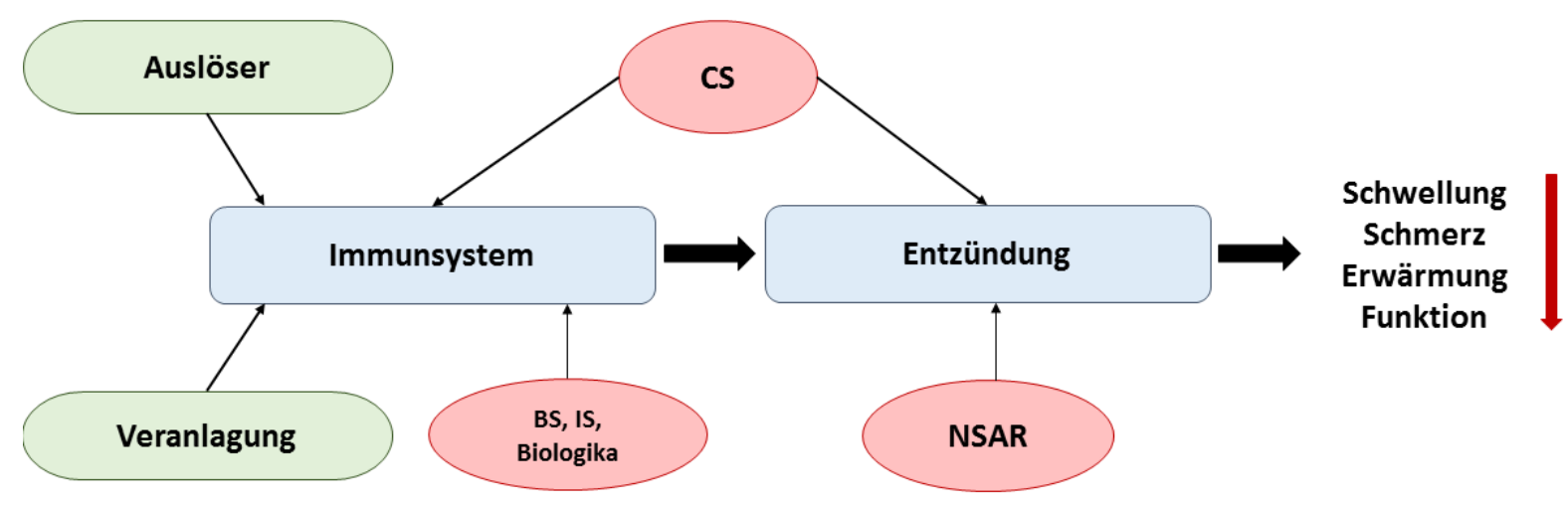

$\mathrm{CS}=$ Cortisonpräparate, $\mathrm{BS}=$ Basistherapeutika, IS = Immunsuppressiva/Zytostatika, NSAR = nichtsteroidale Antirheumatika

Abb. 3: Modulierende Faktoren des Krankheitsgeschehens, eigene Darstellung modifiziert nach Kinderklinik Garmisch-Partenkirchen gGmbH - Deutsches Zentrum für Kinder- und Jugendrheumatologie (2012, S.47) 


\subsubsection{Beschreibung der Medikamentengruppen}

\subsection{Nichtsteroidale Antirheumatika (NSAR)}

Bei den NSARs handelt es sich um die am häufigsten verwendeten Medikamente für die Behandlung einer JIA. Insbesondere bei der Oligo-und der Polyarthritis beginnt die medikamentöse Therapie oftmals mit nichtsteroidalen Antirheumatika. Diese wirken sowohl entzündungshemmend (antiphlogistisch) als auch schmerzstillend (analgetisch) über eine Blockade der Prostaglandinsynthese (Harris et al. 2013). Ibuprofen, Naproxen, Indometacin und Diclofenac gehören zu den am häufigsten verwendeten NSAR's (Heubner et al. 2002).

\subsection{Basismedikamente, nicht-biologische DMARDs}

Bei Basismedikamenten, auch DMARDs genannt, handelt sich um Substanzen, die in erster Linie den Krankheitsverlauf positiv beeinflussen sollen, anstatt nur die Symptome wie Schmerzen, Schwellungen usw. zu unterdrücken. Basismedikamente beeinflussen und schwächen mit verschiedensten Mechanismen die Reaktionen des Immunsystems. Methotrexat (MTX) ist das am häufigsten eingesetzte Basistherapeutikum und bewirkt eine kompetitive Hemmung der DihydrofolatReduktase, einem Schlüsselenzym in der DNA-Synthese (Beukelman et al. 2012). Ein Wirkungsmechanismus bei der Beeinflussung von Entzündungsreaktionen ist die Freisetzung von Adenosin, was nachfolgend die T-Zell-Aktivierung inhibiert (Kumar und Banik 2013). Weitere Basistherapeutika, welche oftmals bei Unwirksamkeit oder Unverträglichkeit des MTX eine effektive Alternative darstellen, sind das Leflunamid, das Azathioprin oder das Sulfaszalazin (Foeldvari und Wierk 2010). Diese Medikamente wirken ebenfalls hauptsächlich über eine Beeinträchtigung der Lymphozyten-funktionen (Meier et al. 2013, Reutter-Simon 2013). Die Verwendung des Antimalariamedikamentes Hydroxychloroquin wird bei eher milden Formen einer aktiven RA beschrieben (Kumar und Banik 2013). Hydroxychloroquin kommt aber vor allem bei den Kollagenosen zum Einsatz und wird oft in Kombination mit MTX angewandt (Reutter-Simon 2013).

\subsection{Glukokortikoide}

Glukokortikoide besitzen eine sehr gute antiphlogistische Wirkung und gelten als die effektivsten Medikamente in der Rheumatherapie. Dem stehen allerdings eine Vielzahl schwerer Nebenwirkungen gegenüber (Heubner et al. 2002). Kortikoste- 
roide wirken stark antiphlogistisch und antiödematös. Kortison hemmt neben der Cyclooxygenase auch innerhalb der Mastzellen die Expression von Botenstoffen, wie Interleukin-1-beta (IL1- $\beta$ ) und Tumornekrosefaktor-alpha (TNF- $\alpha$ ).

\subsection{Biologika, biologische DMARDs}

Im Laufe der letzten 15 Jahre wurden zahlreiche neue Substanzen dieser Medikamentengruppe entwickelt, was eine neue Ära in der Behandlung der JIA eingeläutet hat (Meier et al. 2013). Seitdem stehen u. a. TNF-, IL-1- und IL-6-Inhibitoren zur Verfügung. Bisher als TNF-alpha-Inhibitoren etablierte Substanzen sind z. B. Etanerzept, Adalimumap und Infliximap. Im Weiteren stellt der IL-6Rezeptorantagonist Tocilizumab eine sinnvolle Behandlungsoption bei der refraktären RA dar (Emery et al. 2008).

\subsubsection{Orale Nebenwirkungen der Medikamente}

Die Medikamenteneinnahme bei JIA-Patienten gilt als ein Einflussfaktor, der sowohl lokal als auch systemisch orale Erkrankungen bei Patienten begünstigen kann. Zahlreiche Medikamente werden in einer zuckerhaltigen Darreichungsform verabreicht, um die Akzeptanz zur Medikamenteneinnahme bei den jungen Patienten zu erhöhen (Bhatt et al. 2014). Es wird eine erhöhte Kariesanfälligkeit bei der regelmäßigen Gabe zuckerhaltiger Medikamente beschrieben (Shaw und Glenwright 1989). Fabri et al. (2014) beschreiben die Medikamenteneinnahme daneben als bedeutendsten Risikofaktor bei der Entstehung parodontaler Entzündungen. Immunsuppressiva, wie Cyclosporine, können vermehrt gingivale Wucherungen, Ulzerationen oder gingivale Blutungen hervorrufen (Lucas und Roberts 2005, Seymour 2003). In einer Tierstudie konnte gezeigt werden, dass ein lang anhaltender Einsatz von MTX eine Destruktion des Alveolarknochens nach sich ziehen kann (Yoshinari et al. 1994). Glukokortikoide können möglicherweise eine osteoporoseinduzierende Wirkung haben (Seguro et al. 2013). Eine verringerte Wundheilung und eine erhöhte Anfälligkeit gegenüber gingivalen Infektionen sind ebenfalls möglich. Ob die Medikation bei JIA-Patienten einen Effekt auf den Alveolarknochen hat, ist bisher aber noch unklar (Fabri et al. 2014). Das Auftreten einer Parodontitis unter einer immunsupprimierenden Therapie wird eher selten beschrieben. Bei JIA-Patienten, welchen TNF-Inhibitoren verabreicht werden, konnten erhöhte Sondierungstiefen bei geringem Bleeding on Probing (BOP) festgestellt werden (Fabri et al. 2014). 


\subsubsection{Symptome und Funktionseinschränkungen bei JIA}

Allen Verlaufsformen der JIA gemeinsam ist das Symptom Arthritis. Die entzündliche Reaktion führt zu einer gesteigerten Durchblutung und zu einem Anschwellen der Gelenkinnenhaut. Es kommt zu einer vermehrten Bildung von Gelenkflüssigkeit, dem so genannten Erguss. Das Gelenk schwillt an und schmerzt. Es können sich Flüssigkeitsansammlungen in den gelenknahen Schleimbeuteln bilden, sog. Synovialzysten. Diese treten vor allem in den Kniegelenken (Bakerzysten) aber auch an der Schulter oder am Arm auf (Alessi et al. 2012). Bei der Oligoarthritis $(\mathrm{OA})$ sind maximal vier Gelenken während der ersten sechs Erkrankungsmonate betroffen. Dies sind vor allem die großen Gelenke der unteren Extremitäten, wie Knie- und Sprunggelenke. Mittlerweile wird darauf hingewiesen, dass auch die Kiefergelenke sehr häufig betroffen und vielleicht sogar die am häufigsten betroffenen Gelenke darstellen (Koos et al. 2014, Koos et al. 2011). Klinische Symptome sind Schwellungen, Schmerzen, Rötung, Überwärmung und Bewegungseinschränkung. Durch den Entzündungsreiz kann es zu einem asymmetrischen Knochenwachstum mit anfänglichen Wachstumsvorteilen des betroffenen Gelenkes kommen (Reutter und Girschick 2013). Allerdings ist zu beobachten, dass die jungen Patienten häufig keine Schmerzsymptomatik äußern, sodass eine Morgensteifigkeit und generelle Untätigkeit zur Schonung betroffener Gelenke erste Krankheitsanzeichen darstellen (Hahn und Kimm 2010).

Bei der Polyarthritis (PA) erkranken definitionsgemäß mehr als vier Gelenke. Es kommt in der Regel zu einem symmetrischen Befall sowohl kleiner als auch groBer Gelenke. Typischerweise finden sich Schwellungen der Fingergrund- und mittelgelenke sowie des Weiteren auch der Handgelenke (Reutter und Girschick 2013, Hahn und Kimm 2010).

\subsubsection{Der Kiefergelenkbefall}

Bei JIA-Patienten stellen die Kiefergelenke bei einer Mitbeteiligung in bis zu $87 \%$ der Fälle die wohl am häufigsten betroffenen Gelenke dar (Tzaribachev et al. 2010). Mädchen sind hierbei häufiger betroffen als Jungen. Das Risiko einer Beteiligung der Kiefergelenke steigt bei einem sehr frühen Krankheitsbeginn. Bei lang andauernden und schweren Krankheitsverläufen muss ebenfalls verstärkt mit einer Beteiligung der Kiefergelenke gerechnet werden (Deutsche Rheuma-Liga Bundesverband 2016). Eine Besonderheit der Kiefergelenke ist, dass sich das 
Wachstumszentrum des Unterkiefers im Bereich der Kondylen befindet. Entzündliche Vorgänge können demnach zu Störungen der wachstumsmodulierenden Prozesse führen (Weiss et al. 2008). Bei symptomatischen Verläufen sind früh auftretende Symptome wie Schmerzen beim Kauen, Kopfschmerzen und eine asymmetrische Mundöffnung möglich (Tzaribachev et al. 2010). Allerdings wird auf die Problematik eines symptomlosen Verlaufes der Kiefergelenkarthritis bei mehr als der Hälfte der Patienten hingewiesen. So kommt es in bis zu 69\% zu asymptomatischen Verläufen. Eine Ursache hierfür könnte zum einen der ungünstige Zugang zur diagnostischen Palpation sein, welcher sich aus der verborgenen Lage und der besonderen Anatomie der Kiefergelenke ergibt. Des Weiteren werden Schmerzen in diesem Gelenk oftmals nicht wahrgenommen, da Kinder im Vergleich zu Erwachsenen eine hohe Schmerzschwelle aufweisen (Tzaribachev et al. 2010). Der weitere Krankheitsverlauf kann durch Funktionsstörungen und Bewegungseinschränkungen, wie bspw. einer eingeschränkten Mundöffnung, geprägt sein (Tzaribachev et al. 2010). Die Zerstörung von Kondylen, Diskus und Bandapparat sind drastische Folgen entzündlicher Vorgänge in den Kiefergelenken (KahlNieke 2013, Tzaribachev et al. 2010). Ebenso können eine Mikro- und Retrognathie der Mandibula sowie eine mandibuläre Posteriorrotation des Unterkiefers mit resultierendem offenem Biss resultieren (Carvalho et al. 2012).

Die Diagnostik einer Kiefergelenkarthritis bei JIA-Patienten ist gegenüber anderen Gelenken erschwert (Carvalho et al 2012). Insbesondere in frühen Erkrankungsstadien, aber auch bei fortgeschrittener Kiefergelenkarthritis, fehlen oftmals subjektiv wahrgenommene und klinische Auffälligkeiten (Cedströmer et al. 2014, Svensson et al. 2001). Die Untersuchung muss sehr sorgfältig und präzise erfolgen, wobei rheumaspezifische Anamnese- und Untersuchungsbögen verwendet werden sollten (Dāvidsone et al. 2016, Kahl-Nieke 2013, Hiz et al. 2012). Die klinische Diagnostik alleine ist allerdings nicht ausreichend kondyläre Veränderungen zu diagnostizieren (Hu et al. 2009). Unterstützend kommen bildgebende Verfahren, wie das OPG, die kontrastmittelverstärkte Magnetresonanztomographie (MRT) oder sonographische Verfahren zum Einsatz (Kahl-Nieke 2013). Das MRT gilt in der Kiefergelenksdiagnostik als Goldstandard, wobei ein bedeutender Nachteil in der notwendigen Sedierung der jungen Patienten liegt (Cedströmer et al. 2014, Argyropoulou et al. 2009, Twilt et al. 2008). Zur Evaluation starker Diagnostikparameter zählen neben anamnestischen und klinischen Auffälligkeiten auch 
weitere Parameter, wie Erkrankungsart und -aktivität, Patientenalter, Blutparameter und genetische Aspekte (Dāvidsone et al. 2016, Niibo et al. 2016, Keller et al. 2015, Cedströmer et al. 2014, Abramowicz et al. 2013, Koos et al. 2011, Argyropoulou et al. 2009, Weiss et al. 2008, Billiau et al. 2007, Twilt et al. 2004).

\subsubsection{Extraartikuläre Manifestationen}

Die Uveitis, eine entzündliche Erkrankung der Augen, stellt die häufigste extraartikuläre Manifestation der JIA dar (Wentworth et al. 2014). Des Weiteren können Krankheitszeichen wie Sehnenscheidenentzündungen (Enthesitis) und Schuppenflechte (Psoriasis) hinzukommen (Reutter und Girschick 2013). Herpes Zoster konnte ebenfalls gehäuft bei JIA-Patienten festgestellt werden. Zusammenhänge wären aufgrund der Grunderkrankung und auch durch die Einnahme immunmodulierender Medikamente denkbar, wie u. a. Etanercept oder Kortikosteroide (Nimmrich und Horneff 2015). Bei an Arthritis erkrankten Kindern konnte gleichzeitig eine deutlich erhöhte Belastung durch psychosoziale Stressfaktoren beobachtet werden (Neufeld et al. 2013, Russo et al. 2012). Laut einer groß angelegten Studie im Jahre 2012, leiden 16\% der JIA-Patienten an ernsthaften psychischen Störungen (Pohjankoski et al. 2012). Stressbedingt kann es zu einem Anstieg proinflammatorischer Zytokine und Cortisol kommen, was eine Assoziation erklären könnte (Sävendahl 2012). Unter den verschiedenen Subgruppen zeigt sich zudem ein schlechter Allgemeinzustand vor allem bei der systemischen Arthritis. Ein typisches Zeichen der systemischen Form ist Fieber, häufig begleitet von Exanthemen. Es gilt als sicher, dass insbesondere die systemische Form der JIA bei zahlreichen Patienten mit Erkrankungen des Herzens, wie beispielsweise einer Perikarditis oder Myokarditis, vergesellschaftet ist (Alkady et al. 2012, Goldsmith 2012). Eine finnische Studie zeigte zudem, dass bei JIA-Patienten eine gleichzeitige Erkrankung an Diabetes mellitus Typ 1 um bis zu 4,5mal erhöht war (Pohjankoski et al. 2012). Weiterhin kann es bei der systemischen JIA zur Lymphadenopathie, Hepatosplenomegalie und zu Hautausschlägen kommen (Kadavath und Efthimiou 2015). Schwerwiegende Komplikationen stellen die Amyloidose sowie das Makrophagenaktivierungssyndrom (MAS) dar (Kadavath und Efthimiou 2015). Die Folge dieser Erkrankungen ist eine zunehmende Organinsuffizienz (Isenberg et al. 2004). Das MAS ist eine wenn auch seltene aber lebensbedrohliche Komplikation (Reutter und Girschick 2013). 


\subsection{Zusammenhänge zwischen der JIA und oralen Erkrankungen}

Eine Assoziation zwischen der JIA und oralen Erkrankungen wird kontrovers diskutiert. Während in einigen Publikationen signifikante Zusammenhänge zwischen der JIA und der Karies beschrieben werden (Leksell et al. 2008, Savioli et al. 2004, Welbury et al. 2003), konnten andere kein erhöhtes Kariesvorkommen bei JIA-Patienten feststellen (Feres de Melo et al. 2014, Ahmed et al. 2004). Über ein vermehrtes Auftreten von Gingivitis bei JIA-Patienten wurde in einigen Studien berichtet (Ahmed et al. 2004, Savioli et al. 2004, Welbury et al. 2003). Zusammenhänge der JIA mit der Parodontitis wurden allerdings selten beobachtet (Hucke 2014).

Es existieren zahlreiche mögliche Begründungen für eine Assoziation der JIA mit Karies oder der Gingivitis bzw. Parodontitis (Bhatt et al. 2014, Leksell et al. 2008, Savioli et al. 2004, Welbury et al. 2003, Walton et al. 2002, Walton et al. 2000). Walton et al. (2000) beschrieben eine erschwerte Mundhygiene bei JIA-Patienten, welche bei einer Mitbeteiligung von Kiefer-, Finger- oder Handgelenken eine verminderte Beweglichkeit aufwiesen. Klinisch äußern sich Entzündungen dieser Gelenke in einer eingeschränkten Mundöffnung und einer erheblich eingeschränkten Greiffunktion der Hände (Walton et al. 2000). Die hierdurch erschwerte Mundhygiene kann die Biofilmakkumulation und das Auftreten von parodontalen Erkrankungen und Karies begünstigen (Barr et al. 2008, Savioli et al. 2004). Eine erhöhte Biofilmakkumulation könnte auch durch eine veränderte Ernährungsweise gefördert werden. Es gibt Hinweise darauf, dass erkrankte Patienten über den Tag verteilt mehrere kleine und zuckerhaltige Mahlzeiten zu sich nehmen und damit die tägliche Zuckerexposition erhöht ist (Tanchyk 1991). Eine erhöhte Zuckerexposition kann auch in der Gabe von Medikamenten begründet sein. Diese liegen häufig in einer zuckerhaltigen Darreichungsform vor, um die Akzeptanz zur Medikamenteneinnahme bei den jungen Patienten zu erhöhen (Bhatt et al. 2014). Weitere Auswirkungen JIA-relevanter Medikamente wurden bereits unter Punkt 3.3.3.2 erläutert.

Gründe für gehäuft auftretende orale Erkrankungen bei JIA-Patienten könnten auch eine geringere Speichelproduktion sowie veränderte Konzentrationen antimikrobieller Speichelproteine sein (Walton et al. 2002, Siamopoulou et al. 1989). Weiterhin ist zu beachten, dass es bei Patienten mit rheumatoider Arthritis aufgrund der als oftmals wichtiger angesehenen Rheuma-Therapie eher zur Vernach- 
lässigung der zahnärztlichen Behandlung kommt, was orale Defizite zusätzlich begünstigen kann (Deschner 2011).

Systemische Zusammenhänge zwischen der Parodontitis und der rheumatoiden Arthritis wurde bei Erwachsenen weit besser untersucht als bei Kindern (Calderaro et al. 2017, Holmstrup et al. 2017, Tang et al. 2017, Kaur et al. 2013). Hierbei zeigen sich zum einen mehr Zahnverlust bei Rheumapatienten sowie ein häufigeres Auftreten insbesondere schwerer PD-Formen. Zum anderen konnten Ortiz et al. (2009) bei Patienten mit rheumatoider Arthritis beobachten, dass bei Behandlung einer vorliegenden Parodontitis durch eine Zahnreinigung und antiinfektiösen Maßnahmen nicht nur die Mundgesundheit verbessert, sondern ebenfalls Schmerzen und Zahl der geschwollenen Gelenke verringert wurde. Umgekehrt kann durch die Therapie der rheumatoiden Arthritis mit DMARDs/Biologika die parodontale Situation verbessert werden (Deschner 2011). Hierbei könnte es sich jedoch auch um einen direkten Effekt der Medikamente auf das Parodont handeln (Mayer et al. 2009). Auch neuere Studien mit Rheumapatienten bestätigen einen Rückgang zahlreicher Entzündungsparameter, wie beispielsweise dem CRP, nach der Durchführung einer parodontalen Therapie (Erciyas et al. 2013). Die Studienlage gibt daher Anlass, bei Rheumapatienten in besonderem Maße auf die Mundhygiene/-gesundheit zu achten (Straka et al. 2012).

Für die PD wie die RA konnten gemeinsame Risikofaktoren wie Rauchen oder Übergewicht identifiziert werden (Persson 2012). Eine Begründung für systemisch auftretende Wechselwirkungen zwischen einer vorliegenden PD und rheumatoiden Erkrankungen sind auffallend ähnliche, pathologische Prozesse. In Tabelle 2 sind die Gemeinsamkeiten zwischen den beiden Erkrankungen (PD und RA) aufgeführt. 
Tab. 2: Gemeinsamkeiten von Parodontitis und rheumatoider Arthritis

\begin{tabular}{|c|c|}
\hline Rheumatoide Arthritis & Parodontitis \\
\hline chronisch-entzündliche Erkrankung & chronisch-entzündliche Erkrankung \\
\hline schubweiser Verlauf & schubweiser Verlauf \\
\hline Ausgangsgewebe: Membrana synovialis & Ausgangsgewebe: Parodontium \\
\hline multifaktorielle Pathogenese & multifaktorielle Pathogenese \\
\hline bakterielle Infektion als Auslöser wird diskutiert & $\begin{array}{l}\text { bakterielle Infektion mit gram-negativen, anae- } \\
\text { roben Keimen ist Voraussetzung für die Er- } \\
\text { krankung }\end{array}$ \\
\hline $\begin{array}{l}\text { Gewebedestruktion durch autoaggressive Im- } \\
\text { munreaktion }\end{array}$ & $\begin{array}{l}\text { Gewebedestruktion durch autoaggressive Im- } \\
\text { munreaktion }\end{array}$ \\
\hline $\begin{array}{l}\text { Risikofaktoren sind bekannt (z. B. Nikotinabu- } \\
\text { sus, Übergewicht) }\end{array}$ & $\begin{array}{l}\text { Risikofaktoren sind bekannt (z. B. Nikotinabu- } \\
\text { sus, Übergewicht, Stress, Medikamentenein- } \\
\text { nahme, Mundhygiene) }\end{array}$ \\
\hline $\begin{array}{l}\text { Assoziation mit systemischen Erkrankungen } \\
\text { (z. B. koronare Herzerkrankungen, Nierener- } \\
\text { krankungen, Osteoporose) }\end{array}$ & $\begin{array}{l}\text { Assoziation mit systemischen Erkrankungen } \\
\text { (z. B. koronare Herzerkrankungen, Diabetes } \\
\text { mellitus, Nierenerkrankungen) }\end{array}$ \\
\hline genetische Prädisposition ist bekannt & genetische Prädisposition wird diskutiert \\
\hline
\end{tabular}

Immunpathologische Prozesse führen bei den Krankheitsverläufen der PD und der RA zur Zerstörung von Bindegewebe und Knochen (Routsias et al. 2011, Detert et al. 2010). Erhöhte Zytokin- und Tumornekrosefaktor-alpha-Werte (TNF- $\alpha$ ) werden sowohl bei der PD als auch der RA bzw. der JIA beobachtet (Arunachalam 2014, El-Shinnawi und Soory 2013). Zytokine führen letztlich durch die Aktivierung von Matrixmetalloproteinasen (MMPs) zur Knorpel- und Knochendestruktion in den Gelenken und im Parodont zur Zerstörung des Alveolarknochens (Dev et al.

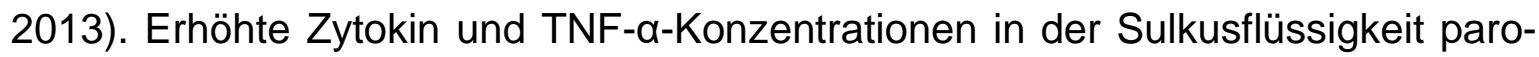
dontal gesunder Rheumapatienten zeigen, dass die Pathogene einer Erkrankung in die strukturellen Gegebenheiten der jeweils anderen Erkrankung vordringen können (Javed et al. 2014). Ähnlich der Zytokine konnten bei JIA- und PDPatienten auch gemeinsame Entzündungsmarker wie Neutrophile, Leukozyten, das CRP sowie Rheumafaktoren gefunden werden (Kanaparthy et al. 2012, Mizuno et al. 2011, Minden 2009, Havemose-Poulsen et al. 2006). Zudem wird bei erwachsenen RA-Patienten von einer Mitbeteiligung parodontitisassoziierter $\mathrm{Pa}$ thogene an dem Krankheitsgeschehen berichtet (Białowąs et al. 2014, Mikuls et al. 2014, Kinloch et al. 2008). Dabei besitzt bspw. Porphyromonas gingivalis das 
Potenzial über citrullinierte Peptide oder Hitzeschockproteine immunpathologische Körperreaktionen zu beeinflussen (Persson 2012). Bei Kindern, insbesondere JIAPatienten, wurden derartige Zusammenhänge jedoch noch nicht beobachtet. Allerdings werden bei der RA wie auch bei der JIA genetische Assoziationen zur PD angenommen. Insbesondere wurden HLA-Assoziationen der Klasse I und II beobachtet (Reichert et al. 2007, Van Dyke et al. 2005). Diese Gene kodieren Oberflächenmoleküle antigenpräsentierender Zellen. Bei Erwachsenen konnten zwischen der PD und der RA genetische Zusammenhänge zu weiteren Erkrankungen, wie u. a. koronarer Herzerkrankungen oder Osteoporose gefunden werden (Persson 2012). Abbildung 4 zeigt eine Übersicht möglicher Zusammenhänge, welche zwischen der Parodontitis und dem Beispiel der RA diskutiert werden.

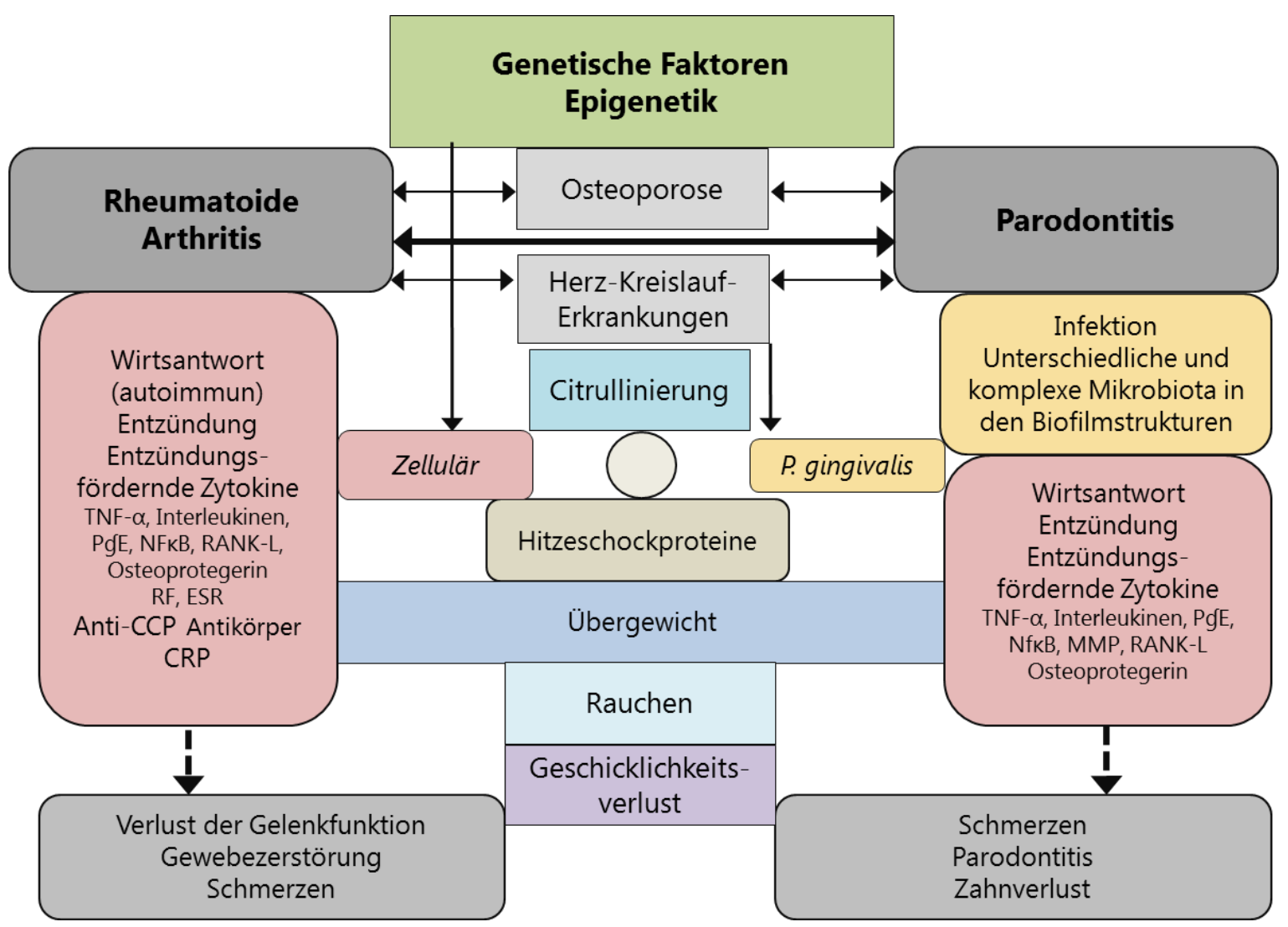

Abb. 4: Darstellung genetischer Faktoren und entzündlicher Assoziationen zwischen Parodontitis und rheumatoider Arthritis, eigene Darstellung modifiziert nach Persson (2012, S.13)

Auch wenn zahlreiche Begründungen für eine Assoziation der JIA mit der PD genannt werden, bleibt der pathogenetische Zusammenhang letztlich bisher ungeklärt (Hucke 2014). 


\section{Material und Methode}

\subsection{Studientyp}

Bei dieser Untersuchung handelt es sich um eine klinische Querschnittsstudie zum Mundgesundheitszustand und der Befundung von Kiefergelenken bei Kindern und Jugendlichen mit juveniler idiopathischer Arthritis. Die Untersuchungen wurden von der Ethikkommission der Medizinischen Fakultät der Universität Göttingen unter der Antragsnummer 3/3/13 geprüft und bewilligt. Alle Patienten wurden vorab schriftlich sowie mündlich über das Studienvorhaben informiert (siehe Anhang 10.3). Die Teilnahme an der Untersuchung war freiwillig und wurde schriftlich bestätigt (siehe Anhang 9.4).

\subsection{Patienten}

Im Rahmen einer rheumatologisch-interdisziplinären Diagnostik erfolgte in der Poliklinik für Präventive Zahnmedizin, Parodontologie und Kariologie der Universitätsmedizin Göttingen routinemäßig und standardisiert eine konsiliarische, zahnärztliche Untersuchung stationärer JIA-Patienten. Zudem wurden JIA-Patienten mit Verdacht einer Mitbeteiligung der Kiefergelenke aus der Praxis für Kinderheilkunde und Kinderrheumatologie des niedergelassenen Kinderarztes Dr. Michael Rühlmann zum zahnärztlichen Konsil in die Klinik überwiesen. Bei der zahnärztlichen Konsiluntersuchung wurden die Patienten um freiwillige Teilnahme an der Studie gebeten.

Für die vorliegende Studie wurden die Daten sowohl aus der zahnärztlichen als auch der allgemeinmedizinischen Untersuchung ausgewertet.

Aufgrund des JIA-Patientenaufkommens im Zentrum für Kinderheilkunde und Jugendmedizin der Universitätsmedizin Göttingen und der externen Praxis für Kinderheilkunde und Kinderrheumatologie (s. o.: Untersuchungszentren) mit maximal ein bis drei konsiliarischen Patienten wöchentlich konnten im Untersuchungszeitraum von November 2012 bis September 2014 insgesamt 59 JIA-Patienten (Kinder und Jugendliche) gewonnen werden.

Folgende Einschlusskriterien wurden definiert:

- Teilnehmer sind sowohl weiblich als auch männlich

- Alter: 6 - 18 Jahre 
- Patienten mit diagnostizierter juveniler idiopathischer Arthritis

- Einwilligung durch Patienten und erziehungsberechtigter Personen

- freiwillige Teilnahme vorausgesetzt

Folgende Ausschlusskriterien wurden formuliert:

- undurchführbare orale Untersuchung aufgrund schlechten Allgemeinzustandes

- andere immunsupprimierte Patienten

- organtransplantierte Patienten

- Vorliegen von Hepatitis A, B, C, TBC, HIV (auf Nachfrage)

- Vorliegen weiterer Allgemeinerkrankungen, wie z. B. Diabetes mellitus

- Patienten mit Anfalls- oder Nervenleiden

- bestehende Schwangerschaft (auf Nachfrage)

- Patienten mit Niereninsuffizienz/Notwendigkeit einer Endokarditisprophylaxe

- nicht auf die JIA zurückzuführende Einschränkungen in der Feinmotorik

- Antibiotikaeinnahme: < 3 Monate

Sonstige Ausschlusskriterien existierten nicht.

\subsection{Allgemeine Datenerfassung}

Um an studienrelevante Patienteninformationen zu gelangen, wurden verschiedene Informationen zum klinischen Ausgangszustand des Patienten erfasst. Hierfür erfolgte eine Datenerfassung durch Auswertung vorliegender medizinischer Befunde- und Behandlungsdokumentationen des Zentrums für Kinderheilkunde und Jugendmedizin der Universitätsmedizin Göttingen sowie der externen Praxis für Kinderheilkunde und Kinderrheumatologie Dr. Michael Rühlmann. Für alle Patienten wurden folgende Parameter erfasst:

- Geschlecht und Alter

- Allgemeinanamnese: Erkrankung und Medikation (sowohl aktuelle Medikation zum Untersuchungszeitraum als auch vergangene Phasen von Medikamenteneinnahmen)

- Zeitpunkt der Diagnosestellung der juvenilen idiopathischen Arthritis

- Stadium der Erkrankung (Progression, Stagnation) sowie Medikation

- ggf. vorhandene Blutparameter 
- CRP (In Anlehnung an Argyropoulou et al. (2009) und den Vorgaben des Uniklinikums Göttingen wurde ein erhöhter CRP ab >8mg/l angenommen.

- Antinukleäre Antikörper (ANA)

- Rheumafaktoren (RF)

○ Histokompatibilitätsantigen HLA-B27

\subsubsection{Anamnese- und Funktionsfragebogen}

Im Weiteren wurde den Patienten ein Anamnesebogen (siehe Anhang 9.1) und ein speziell für die Studie entwickelter Funktions- bzw. Schmerzfragebogen (siehe Anhang 9.2) ausgehändigt. Der Patient und ggf. die erziehungsberechtigte Person wurden gebeten, die studienrelevanten Unterlagen sorgfältig durchzulesen und die Fragebögen gewissenhaft zu beantworten.

Der Anamnesebogen enthielt Fragen zu Allgemeinerkrankungen und zur Mundgesundheit. Neben Herzkreislauferkrankungen und regelmäßiger Medikamenteneinnahme wurden unter anderem der letzte Zahnarztbesuch, Temperaturempfindlichkeit der Zähne, Zahnfleischbluten, Stellungsveränderungen der Zähne, ein schlechter Geschmack im Mund, Zahnlockerung und Zahnfleischbeschwerden, sowie der Nikotinkonsum erfragt.

In den für die Studie entwickelten Funktions- und Schmerzfragebögen wurden die Patienten gebeten, Fragen zu Auffälligkeiten bzw. Schmerzen im Mund-KieferGesichtsbereich (MKG) zu beantworten. In dem Funktionsfragebogen handelte es sich um Fragen zu Bewegungseinschränkungen des Unterkiefers, der Vermeidung harter Speisen, Gelenkgeräuschen sowie dem Pressen und Knirschen mit den Zähnen. Des Weiteren wurden durch den Funktionsfragebogen Ohrgeräusche und ein unbequemer Zusammenbiss der Zähne erfragt. Im Schmerzfragebogen wurden Schmerzen im MKG-Bereich, deren Lokalisation, Intensität, Verweildauer und Zeitpunkt des Auftretens sowie ggf. zurückliegende Traumata erfragt.

\subsection{Klinisch-zahnmedizinische Untersuchung}

In der zahnärztlichen Untersuchung wurden ein zahnärztlicher (dmf-t/DMF-T), parodontaler Befunde (PSI und $\mathrm{PBI}$ ) sowie ein funktionsdiagnostischer Befund (RDC/TMD) erhoben. Die Untersuchungen wurden im Rahmen eines standardi- 
sierten Behandlungsablaufes immer durch den gleichen Behandler durchgeführt. Für die Querschnittstudie wurden die Befundunterlagen und, falls vorhanden, aktuelle Röntgenbilder (OPG) ausgewertet.

\subsubsection{Zahnärztlicher Befund (DMF-T)}

Der DMF-T-Index ist ein international anerkannter Index zur Messung der Karieserfahrung bei einer Einzelperson oder der Kariesverbreitung in einer Bevölkerungsgruppe. Der DMF-T-Index beurteilt die Anzahl der bleibenden Zähne, welche aufgrund von Karies zerstört (Decayed), extrahiert (Missing) oder gefüllt (Filled) wurden (Klein und Palmer 1938). Bei den extrahierten Zähnen ist zu beachten, dass nur Zähne in den Index mit einberechnet werden, welche offensichtlich in Folge einer kariösen Zerstörung verloren gegangen sind. Zähne, deren Verlust auf die normale Exfoliation im Wechselgebiss zurückzuführen ist, sowie Weisheitszähne werden nicht mitgezählt. Im Milchgebiss wird der dmf-t-Index berechnet. Da Weisheitszähne nicht mitgerechnet werden, kann der DMF-T maximal einen Wert von 28 und der dmf-t maximal einen Wert von 20 annehmen. Die Patienten der vorliegenden Studie befanden sich zum Großteil in der Phase des Wechselgebisses. Hier können sowohl Milchzähne als auch bleibende Zähne vorhanden sein. Bei diesen Patienten wurde der gemischte dmf-t/DMF-T erhoben. Dies bedeutet, dass der dmf-t an den vorhandenen Milchzähnen ermittelt und bleibende Zähne dann mit einbezogen wurden, wenn sie sich mindestens zur Hälfte im Durchbruch befanden.

\subsubsection{Parodontale Befunde}

\subsubsection{Gingivale Entzündung (PBI)}

Zur Diagnostik gingivaler Entzündungen wurde der Papillen-Blutungsindex nach Saxer und Mühlemann durchgeführt. Durch diesen Index kann insbesondere der Schweregrad einer Gingivitis beurteilt werden (Saxer und Mühlemann 1975). Die Untersuchung erfolgte mittels einer stumpfen Parodontalsonde (PCP/UNC 15, HuFriedy, Chicago, USA). Unter relativer Trockenlegung wurde der Sulkus von der Papillenspitze bis zur Basis in einem Winkel von $45^{\circ}$ zur Zahnachse vorsichtig ausgestrichen. Die Sondierung erfolgte im ersten und dritten Quadranten oral und im zweiten und vierten Quadranten vestibulär. Die Blutung wurde nach ca. 20 Sekunden kontrolliert. Der Papillenblutungsindex wird in 5 Grade nach Art der Blutung eingeteilt (Tab. 3). 
Tab. 3: Übersicht über die fünf Grade des PBI

\begin{tabular}{l|l}
\hline Grad & Definition \\
\hline Grad 0 & keine Blutung \\
Grad 1 & Blutungspunkt \\
Grad 2 & mehrere isolierte Blutungspunkte oder Blutlinie \\
Grad 3 & Ausfüllen des interdentale Dreiecks mit Blut \\
Grad 4 & profuse Blutung nach Sondierung; Blut fließt über den Zahn oder die Gingiva \\
\hline
\end{tabular}

Für die späteren statistischen Auswertungen wurde für jeden Patienten ein Entzündungswert berechnet. Hierfür wurden die gemessenen Blutungsgrade addiert und durch die Anzahl der Messstellen geteilt.

\subsubsection{Parodontaler Screening-Index (PSI)}

Der Parodontale Screening-Index stellt ein klinisches Mittel zur Beurteilung der parodontalen Behandlungsbedürftigkeit dar. Er beruht auf einer Modifizierung des CPITN (Community Periodontal Index of Treatment Needs) (Ainamo et al. 1982). Bei Erwachsenen wird der PSI an allen Zähnen ermittelt. Bei Kindern und Jugendlichen bis zur Vollendung des 18. Lebensjahres beschränkt sich die Befundung auf die Zähne 16, 11, 26, 36, 31 und 46 an jeweils sechs Messpunkten je Zahn (mesio-vestibulär, vestibulär, disto-vestibulär, mesio-oral, oral, disto-oral). Für die standardisierte Befunderhebung wurde die WHO-Sonde der Firma Morita® (Kyoto, Japan) verwendet. Für jeden Zahn wurde das höchste Messergebnis in eine Sechsfeldertafel notiert. Die Tabelle 4 gibt eine Übersicht über die möglichen Messergebnisse (Code 0-4) und deren Definition.

Tab. 4: Übersicht über die parodontalen Befunde des PSI

\begin{tabular}{|c|c|}
\hline Code & Definition \\
\hline Code 0 & $\begin{array}{l}\text { schwarzes Band der Sonde bleibt vollständig sichtbar, kein Zahnstein, keine über- } \\
\text { stehenden Restaurationsränder, keine Blutung }\end{array}$ \\
\hline Code 1 & $\begin{array}{l}\text { schwarzes Band der Sonde bleibt vollständig sichtbar, kein Zahnstein, keine über- } \\
\text { stehenden Restaurationsränder, auf Sondierung tritt eine Blutung auf }\end{array}$ \\
\hline Code 2 & $\begin{array}{l}\text { schwarzes Band der Sonde bleibt vollständig sichtbar, Zahnstein oder defekte Res- } \\
\text { taurationsränder lassen sich feststellen }\end{array}$ \\
\hline Code 3 & $\begin{array}{l}\text { schwarzes Band der Sonde bleibt nur teilweise sichtbar, zuvor genannte Befunde } \\
\text { können zusätzlich vorkommen }\end{array}$ \\
\hline Code 4 & $\begin{array}{l}\text { schwarzes Band der Sonde verschwindet vollständig in der Tasche. Hiermit wird eine } \\
\text { Sondierungstiefe gekennzeichnet, die größer als 5,5 Millimeter ist. Die Befunde von } \\
\text { Code } 1 \text { bis } 3 \text { können zusätzlich vorhanden sein }\end{array}$ \\
\hline
\end{tabular}




\subsubsection{Funktionsdiagnostik (RDC/TMD) nach Dworkin und LeResche (1992)}

Die Funktionsdiagnostik wurde standardisiert, nach einem festgelegten Untersuchungsprotokoll der „Research Diagnostic Criteria for Temporomandibular Disorders“ (RDC/TMD) durchgeführt. Die RDC/TMD stellen heute ein bewährtes und validiertes Untersuchungssystem bezüglich temporomandibulärer Erkrankungen dar (Dworkin und LeResche 1992). Die RDC/TMD sind in zwei diagnostische Bereiche, den Achsen I und II, gegliedert. Durch ein klar strukturiertes, standardisiertes Befundschema bezieht sich die Achse I auf die somatische Diagnostik und die Achse II auf eine psychosoziale Diagnostik. In der klinisch zahnmedizinischen Untersuchung der Universitätsmedizin Göttingen wurde ausschließlich die Achse IDiagnostik standardisiert nach der deutschen Version der RDC/TMD (John et al. 2006) durchgeführt und für die vorliegende Studie anhand der Befundblätter (siehe Anhang 10.5) ausgewertet.

Die Befunderhebung umfasste folgende Teilbereiche:

- Befragung zu bestehenden Schmerzen

- Ermittlung der Mundöffnungsbewegung

- Ermittlung des vertikalen Bewegungsumfangs und ggf. dabei auftretender Kiefergelenk- und/oder Muskelschmerzen

- Ermittlung von Gelenkgeräuschen bei Palpation

- Ermittlung des horizontalen Bewegungsumfangs und dabei auftretender Gelenkgeräusch

- Ermittlung von Schmerzen durch Palpation der extra-und intraoralen Muskulatur, sowie der Kiefergelenke

Nachfolgend werden die einzelnen Untersuchungsinhalte kurz beschrieben:

\subsubsection{Mundöffnungsbewegungen}

Der Patient wurde gebeten, den Mund so weit wie möglich zu öffnen. Während der Öffnungsbewegung wurden folgende Bewegungsmuster unterschieden:

- Gerade

- Deflektion: Abweichung des Inzisalpunktes $\geq 2 \mathrm{~mm}$ von der Medianebene

- Deviation: Abweichung des Inzisalpunktes $\geq 2 \mathrm{~mm}$ von der Medianebene mit Rückkehr zur Ebene am Ende der Bewegung

- Typ: Ruckartige oder nicht oben aufgeführte Öffnungsbewegungen 


\subsubsection{Vertikaler Bewegungsumfang des Unterkiefers}

Mit Hilfe eines Lineals wurde der maximale Interinzisalabstand jedes Patienten gemessen. Zusätzlich wurden rechts, links oder beidseitig auftretende Schmerzen bei der Mundöffnung (MÖ) notiert.

- maximale aktive MÖ, ohne Schmerzempfinden des Patienten

- maximale aktive MÖ auch mit Schmerzempfindlichkeit

- maximale passive (unterstützte) MÖ: Nach maximaler MÖ wurde ein mäßiger Druck auf den unteren mittleren Inzisivi angewendet, um den Unterkiefer weiter bis zur Grenzposition zu bewegen.

Des Weiteren wurden der vertikale sowie der horizontale Überbiss der Patienten registriert.

- vertikaler Überbiss (overbite): Bei festem Zusammenbiss wurde mit einem Stift die Stelle markiert, wo die Inzisalkanten der mittleren oberen Inzisivi die unteren Inzisivi am weitesten überlappen. Nach Öffnung der Zähne wurde dieser Wert gemessen.

- horizontaler Überbiss (overjet): Nach festem Zusammenbiss wurde mit einem Lineal der Abstand der Labialfläche der UK-Frontzähne zur Inzisalkante der OK-Frontzähne gemessen.

3.4.3.3 Kiefergelenkgeräusche bei vertikalen, lateralen und protrusiven Unterkieferbewegungen

Für die Ermittlung von Kiefergelenkgeräuschen wurde der Zeigefinger des Behandlers auf dem zu untersuchenden Kiefergelenk des Patienten gelegt, die andere Hand stützte den Kopf ab. Der Patient wurde aufgefordert, den Unterkiefer langsam zu öffnen und zu schließen beziehungsweise nach rechts, links und vorne zu bewegen. Die jeweiligen Bewegungen sollten dreimal wiederholt werden. Auftretende Knack- und Reibegeräusche wurden nach folgender Definition beurteilt:

\footnotetext{
$0=\quad$ kein Geräusch

$1=$ Kiefergelenkknacken: Ein klares Geräusch von kurzer, begrenzter Länge mit deutlichem Anfang und Ende. Ein positiver Vermerk im Untersuchungsbogen erfolgt, wenn das Kiefergelenkknacken bei zwei von drei Unterkieferbewegungen auftritt.
} 
$2=$ grobes Kiefergelenkreiben: Ein kontinuierliches Geräusch im Kiefergelenk über eine längere Periode der Kieferbewegung.

$3=$ feines Kiefergelenkreiben: Ein feines kratzendes Geräusch über eine längere Periode der Kieferbewegung.

Knackgeräusche bei Mundöffnung und -schließung wurden wie folgt eingeteilt:

- reproduzierbares Öffnungsknacken: Knacken während des Öffnens des Mundes aus der maximalen Interkuspidation bei wenigstens zwei von drei Bewegungsabläufen.

- reproduzierbares Schließungsknacken: Knacken während des Schließens des Mundes (aus der maximalen Mundöffnung) bei wenigstens zwei von drei Bewegungsabläufen.

- reproduzierbares reziprokes Knacken: Knacken bei initialer bis intermediärer Öffnung (oder Protrusion) sowie Knacken bei bis terminaler Schließbewegung (oder Retrusion).

Knackgeräusche bei Latero- und Protrusionsbewegungen wurden wie folgt eingeteilt:

- reproduzierbares Knacken: Knacken bei zwei von drei Latero- oder Protrusionsbewegungen.

- nicht-reproduzierbares Knacken: Knacken, welches nicht mindestens zweimal bei drei Latero- oder Protrusionsbewegungen auftrat.

\subsubsection{Unterkiefer-Bewegungen}

- Laterotrusionsbewegung rechts bzw. links: Nach maximalen Laterotrusionsbewegungen wurde mit einem Lineal der Abstand zwischen den Inzisalpunkten der Oberkiefer- bzw. Unterkieferschneidezähne von vestibulär gemessen.

- Protrusionsbewegung: Nach maximaler Protrusion wurde der Interinzisalabstand gemessen. Um die komplette Bewegungsdistanz des Unterkiefers festzuhalten, wurde der zuvor ermittelte Overjet hinzugerechnet.

Rechts- oder linksseitig auftretende Gelenk- oder Muskelschmerzen wurden notiert. 


\subsubsection{Muskelpalpation}

Die Palpation erfolgte mit den Fingerspitzen des Zeige- und Mittelfingers und für jede Seite einzeln. Die Gegenseite des Kopfes wurde mit der anderen Hand gestützt. Der Unterkiefer befand sich in Ruheschwebe. Der Patient sollte mitteilen, ob die Palpation schmerzhaft ist und ob die Schmerzen leicht, mäßig oder stark sind. Missempfindungen wie Druck und/oder Spannungsgefühle wurden nicht gewertet. Die Palpation wurde nach den Richtlinien der RDC/TMD durchgeführt. Eine detaillierte Auflistung der Palpationsstellen findet sich im Anhang 9.5.

\subsubsection{Auswertung konsiliarischer Röntgenbilder}

Im Rahmen der Studie wurden vorliegende digitale OPG-Aufnahmen aus der Konsiluntersuchung ausgewertet. In Anlehnung an vorangegangene röntgenologische Untersuchungen von Arvidsson et al. (2009) und Kahl-Nieke (2013) wurden die Röntgenbilder mittels eines Scoresystems von 0-3 befundet:

- Grad 0: normales, konvex geformtes Gelenk

- Grad 1: geringe Abweichungen des Kondylus von der konvexen Form

- Grad 2: deutlich abgeflachter oder deformierter Kondylus

- Grad 3: fehlen des Kondylus bzw. stark gekürzter, abgeflachter Kondylus

Die Abbildung 5 zeigt eine Auswahl von Kiefergelenkaufnahmen und deren Zuordnung zu den Röntgenscores. 


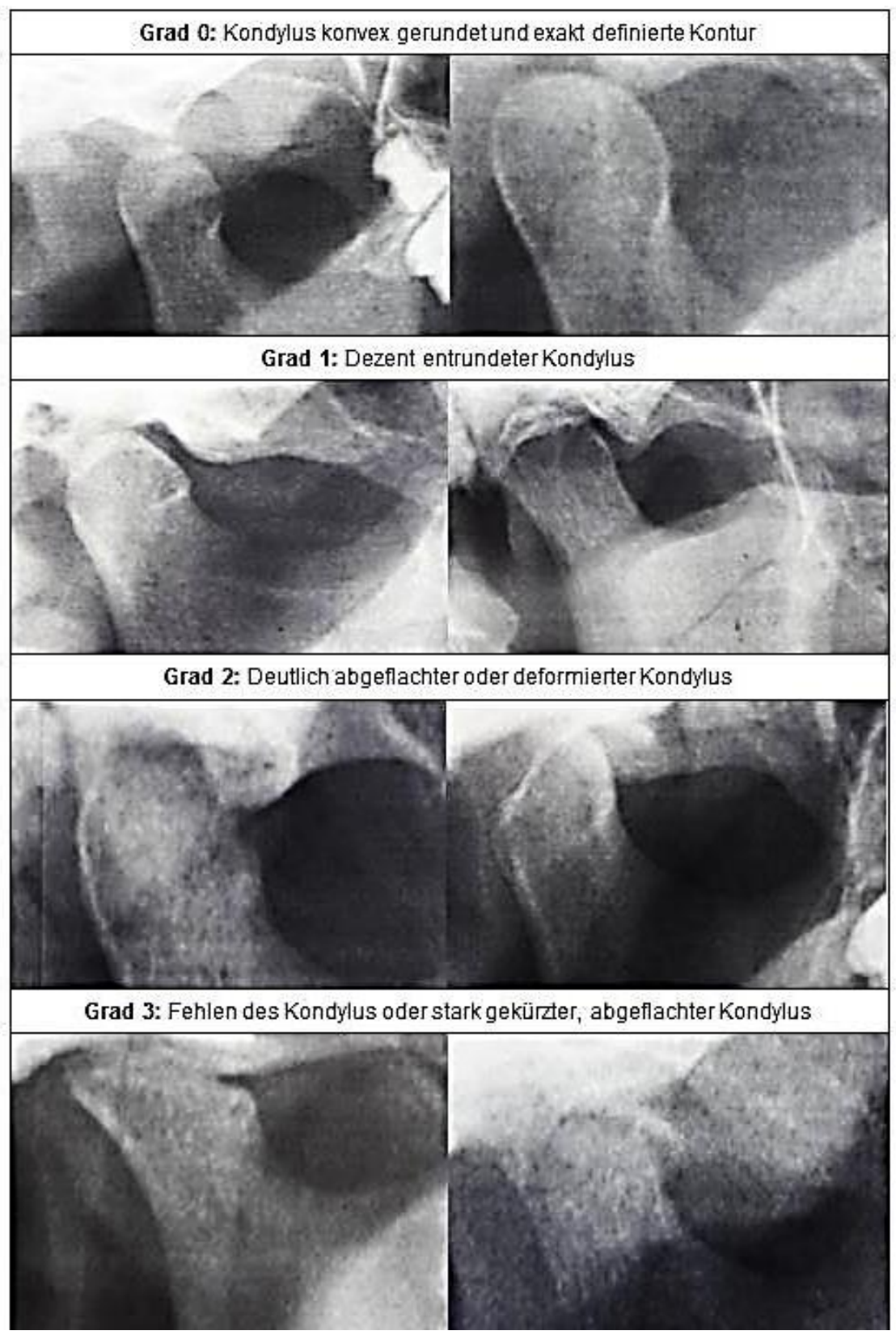

Abb. 5: Definition der vier röntgenologischen Grade, eigene Darstellung

Die Kiefergelenke wurden sowohl rechts- als auch linksseitig einem Score zugeordnet. Da die Zuordnung der Röntgenbilder zu einem Score nicht immer eindeutig war, erfolgte die Befundung der Bilder dreimal in regelmäßigen Abständen immer durch den gleichen Untersucher. Dokumentiert wurde der Score, welcher am häufigsten ermittelt werden konnte. OPGs, bei denen die zu beurteilenden Strukturen mindestens eines Gelenkes nicht abgebildete waren, wurden nicht in die Studie einbezogen. Dies gilt auch für Aufnahmen mit erkennbaren Anomalien der Gelenkstrukturen, welche nicht mit den Definitionen der Röntgenscores zu vereinbaren waren. 


\subsection{Datenverarbeitung und statistische Auswertung}

Alle erhobenen Daten wurden in eine Rohdatentabelle in Microsoft Excel übertragen, kodiert, und nach einer Datenkontrolle ${ }^{1}$ mit Hilfe der Statistiksoftware SPSS Version 19 ausgewertet. Die deskriptive Statistik zur Darstellung von charakteristischen Kenngrößen der Zielgruppe erfolgte über einfache Häufigkeitsanalysen der vorrangig binär-nominal und ordinal kodierten Daten sowie Mittelwert-\& Medianberechnungen der erhobenen stetigen Variablen.

Neben der Beschreibung der oralen Mundgesundheit war in dieser Untersuchung auch die Beziehung zwischen einzelnen erhobenen Variablen von Interesse. Dazu kamen nach Überprüfung der jeweiligen Testvoraussetzungen ausschließlich nichtparametrische Verfahren zum Einsatz. Der Mann-Whitney-U-Test wurde für Gruppenvergleiche zwischen zwei unabhängigen Stichproben oder der KruskalWallis-Test bei Mehrstichprobenproblemen verwendet. Für die Untersuchung von Zusammenhängen und dem Vergleich von Häufigkeitsausprägungen zwischen ordinalen und/oder nominal-binären Variablen wurden Kreuztabellen gewählt. Zur statistischen Absicherung wurde hier auf den $\mathrm{x}^{2}$-Unabhängigkeitstest bzw. falls erforderlich, auf den exakten Test nach Fisher zurückgegriffen. Zur Darstellung der Stärke eines möglichen Zusammenhanges wurde der $\mathrm{x}^{2}$-basierte Kontingenzkoeffizient Cramers-V $(\mathrm{Cl})$ genutzt. Die Analyse von Zusammenhängen zwischen ordinalen und/oder nicht normalverteilten metrischen Variablen erfolgte mittels der Maßzahl Kendalls Tau. Korrelationsanalysen zwischen metrischen Variablen wurden über Produkt-Moment-Korrelationen nach Cohen berechnet.

In Fällen, in denen unterschiedliche Skalenniveaus in eine statistische Analysen eingingen, wurde stets der Test für das niedrigere Skalenniveau gewählt und damit ein konservatives Vorgehen bevorzugt. Das Signifikanzniveau für alle statistischen Tests wurde auf $\alpha<5 \%(p<0,05)$ festgelegt. Da es sich bei den Arbeitshypothesen nur um erste Vermutungen, nicht um bereits empirisch-abgesicherte Erkenntnisse handelt, wurde zudem stets zweiseitig getestet.

1 Vorgenommen über Häufigkeitstest zur Prüfung einer realistischen und vollständigen Dateneingabe. Bei Auffälligkeiten erfolgte ein Abgleich der Kodierungen mit Originalaufzeichnungen (Patientenakten/Forschungsnotizen) und ggf. dem Ausschluss unplausibler Werte durch die Kennzeichnung als fehlender Wert. 


\section{Ergebnisse}

\subsection{Deskriptive Statistik}

\subsubsection{Patientencharakteristik}

Insgesamt konnten 59 Personen $(\mathrm{m}=17 / 29 \%, \mathrm{w}=42 / 71 \%)$ im Alter von 6 - 18 Jahren in die Untersuchung einbezogen werden. Das durchschnittliche Alter der Patienten betrug 12,7 $\pm 3,1$ und die durchschnittliche Erkrankungsdauer 5,1 $\pm 4,5$ Jahre. Die Tabelle 5 gibt einen Überblick über die Patientencharakteristika Alter und Erkrankungsdauer nach Geschlecht.

Tab. 5: Patientencharakteristika Alter und Erkrankungsdauer nach Geschlecht

\begin{tabular}{|c|c|c|}
\hline & $\begin{array}{c}\text { Alter } \\
\text { (in Jahren) } \\
\text { Mittelwert } \pm S A(\min / \max )\end{array}$ & $\begin{array}{l}\begin{array}{c}\text { Erkrankungsdauer } \\
\text { (in Jahren) }\end{array} \\
\text { Mittelwert } \pm S A \text { (min/max) }\end{array}$ \\
\hline $\begin{array}{c}\text { männlich } \\
(n=17,29 \%)\end{array}$ & $12,8 \pm 3,6(8,0 / 17,0)$ & $3,9 \pm 3,0(1,0 / 11,0)$ \\
\hline $\begin{array}{c}\text { weiblich } \\
(n=42,71 \%)\end{array}$ & $12,7 \pm 3,1(6,0 / 18,0)$ & $5,6 \pm 4,9(1,0 / 17,0)$ \\
\hline $\begin{array}{c}\text { gesamt } \\
(n=59,100 \%)\end{array}$ & $12,7 \pm 3,1(6,0 / 18,0)(n=59)$ & $5,1 \pm 4,5(1,0 / 17,0)(n=57)$ \\
\hline
\end{tabular}

Für die Auswertung zu eingenommenen Medikamenten lagen für zwei Patienten keinerlei Angaben zur Medikamenteneinnahme vor. Diese Patienten konnten nicht in die Auswertung mit einbezogen werden. Der überwiegende Teil der Patienten (83\%, $\mathrm{n}=49$ ) hatte bereits Erfahrungen mit der Einnahme von Medikamenten aus der Gruppe der NSAR`s, wie Diclofenac, Ibuprofen oder Naproxen. Eine ebenfalls häufige Medikation bei $46 \%$ der Patienten war das Basistherapeutikum Methotrexat (MTX). Zum Untersuchungszeitpunkt wurde MTX allerdings nur von $12 \%$ $(n=7)$ der Patienten eingenommen (Tab. 6). 
Tab. 6: Eingenommene Medikamente der Studienteilnehmer

\begin{tabular}{|c|c|c|c|}
\hline $\begin{array}{l}\text { eingenommene } \\
\text { Medikamente }\end{array}$ & $\begin{array}{l}\text { insgesamt } \\
\text { Patientenanzahl (\%) }\end{array}$ & $\begin{array}{l}\text { Einnahme während der } \\
\text { Erkrankungsdauer } \\
\text { Patientenanzahl (\%) }\end{array}$ & $\begin{array}{l}\text { Einnahme aktuell } \\
\text { Patientenanzahl (\%) }\end{array}$ \\
\hline NSAR & $n=49(83 \%)$ & k. A. & k. A. \\
\hline Glukokortikoide & $\mathrm{n}=6(10 \%)$ & $\mathrm{n}=2(3 \%)$ & $n=2(3 \%)$ \\
\hline Methotrexat & $n=27(46 \%)$ & $\mathrm{n}=20(34 \%)$ & $\mathrm{n}=7(12 \%)$ \\
\hline Sulfaszalazin & $n=2(3 \%)$ & $n=1(2 \%)$ & $n=1(2 \%)$ \\
\hline $\begin{array}{l}\text { Adalimumap, } \\
\text { Etanercept, } \\
\text { Indometacin }\end{array}$ & $\mathrm{n}=7(12 \%)$ & $n=3(5 \%)$ & $\mathrm{n}=4(7 \%)$ \\
\hline k. $A=$ keine Ang & NSAR = nicht steroi & Antirheumatika & \\
\hline
\end{tabular}

Es lagen nicht bei allen Patienten auswertbare Blutparameter vor (Tab. 7). Auffällig ist, dass nur bei einem Patienten ein positiver Rheumafaktor (RF) getestet wurde.

Tab. 7: Blutparameter der Studienteilnehmer

\begin{tabular}{l|l|l|l}
\hline Blutparameter & $\begin{array}{l}\text { positiv } \\
\text { Patientenanzahl (\%) }\end{array}$ & $\begin{array}{l}\text { negativ } \\
\text { Patientenanzahl (\%) }\end{array}$ & $\begin{array}{l}\text { keine Angabe } \\
\text { Patientenanzahl (\%) }\end{array}$ \\
\hline ANA & $n=31(53 \%)$ & $n=20(34 \%)$ & $n=8(14 \%)$ \\
HLA-B27 & $n=10(17 \%)$ & $n=31(53 \%)$ & $n=18(31 \%)$ \\
RF & $n=1(2 \%)$ & $n=42(71 \%)$ & $n=16(27 \%)$ \\
Anti-DS-DNA & $n=2(3 \%)$ & $n=31(53 \%)$ & $n=26(44 \%)$ \\
CRP & $n=26(44 \%)$ & $n=26(44 \%)$ & $n=7(12 \%)$ \\
\hline
\end{tabular}

positiv: gemessener Blutwert liegt über der Norm, negativ: gemessener Blutwert liegt in der Norm, ANA: Antinukleäre Antikörper, HLA-B27: Human leukocyte antigen B27, RF: Rheumafaktor, AntiDS-DNA: Antikörper gegen Doppelstrang-DNA, CRP: C-reaktives Protein

Die Tabelle 8 gibt einen Überblick über die Verteilung der Erkrankungsart (Oligoarthritis: OA und Polyarthritis: PA) sowie die Mitbeteiligung der einzelnen oberen Gelenke (Finger, Handgelenk, Ellenbogen, Schulter) bei den beiden Erkrankungsarten. Auffällig ist der hohe Anteil an Patienten mit einer Mitbeteiligung der oberen Extremitäten in der Gruppe der PA-Patienten von 86\% $(n=19)$ gegenüber den OA-Patienten mit 30\% ( $n=11)$. 
Tab. 8: Erkrankungsart und mitbeteiligte Gelenke

\begin{tabular}{c|c|c|c|c|c}
\cline { 2 - 6 } & $\begin{array}{c}\text { Finger } \\
\text { Patienten- } \\
\text { anzahl (\%) }\end{array}$ & $\begin{array}{c}\text { Handgelenk } \\
\text { Patienten- } \\
\text { anzahl (\%) }\end{array}$ & $\begin{array}{c}\text { Ellenbogen } \\
\text { Patienten- } \\
\text { anzahl (\%) }\end{array}$ & $\begin{array}{c}\text { Schulter } \\
\text { Patienten- } \\
\text { anzahl (\%) }\end{array}$ & $\begin{array}{c}\text { Sonstiges } \\
\text { Patienten- } \\
\text { anzahl (\%) }\end{array}$ \\
\hline $\begin{array}{c}\text { Oligoarthritis } \\
\mathbf{n = 3 7}\end{array}$ & $\mathrm{n}=6(16 \%)$ & $\mathrm{n}=3(8 \%)$ & $\mathrm{n}=4(11 \%)$ & $\mathrm{n}=0(0 \%)$ & $\mathrm{n}=36(97 \%)$ \\
$\begin{array}{c}\text { Polyarthritis } \\
\mathbf{n = 2 2}\end{array}$ & $\mathrm{n}=15(68 \%)$ & $\mathrm{n}=16(73 \%)$ & $\mathrm{n}=11(50 \%)$ & $\mathrm{n}=3(14 \%)$ & $\mathrm{n}=21(96 \%)$ \\
\hline
\end{tabular}

*obere Extremitäten ausgeschlossen

\subsubsection{Ergebnisse der zahnärztlichen Untersuchung}

Der mittlere dmf-t/DMF-T-Wert der Patienten lag bei 2,6 $\pm 3,0$. Durchschnittlich waren 0,9 $\pm 1,5$ Zähne kariös (d-t/D-T) (Tab. 9). Bezüglich des PBI sind die Durchschnittswerte der einzelnen Patienten nicht normalverteilt. Hier liegt der Median bei 0,44 und der Interquartilsabstand beträgt 0,5 . Die jeweils höchsten PBI-Werte und die höchsten feststellbaren PSI-Werte (PSI-Max) jedes Patienten sind ebenfalls in Tabelle 9 dargestellt. Aufgrund ausgedehnter Multiband-Apparaturen war bei zwei Patienten die Erhebung des PBI und bei zwei Patienten die Erhebung des PBI und des PSI nicht möglich. Lediglich ein Patient wies beim PBI eine absolute Blutungsfreiheit auf. Bei einem großen Teil der Patienten von 34\% $(n=20)$ wurde ein maximaler Wert von 3 und bei immerhin 10\% (n=6) der höchste Wert von 4 gemessen. Lediglich drei Patienten (5\%) waren parodontal gesund (PSI-Score 0). Bei 23 Patienten (39\%) konnte ein maximaler PSI-Wert von 3 gemessen werden. 
Tab. 9: Mittelwerte und Standardabweichungen der Karieserfahrung sowie des maximal gemessenen $\mathrm{PBI}$ und PSI

\begin{tabular}{|c|c|}
\hline \multicolumn{2}{|l|}{ Kariesvorkommen (Mittelwerte $\pm S A$ ) } \\
\hline dmf-t/DMF-T & $2,6 \pm 3,0$ \\
\hline akutes Kariesvorkommen (d-t/D-T) & $0,9 \pm 1,5$ \\
\hline \multicolumn{2}{|c|}{ gingivale Entzündung (maximaler PBI) } \\
\hline PBI 0 & $n=1(2 \%)$ \\
\hline PBI 1 & $n=13(22 \%)$ \\
\hline PBI 2 & $n=15(25 \%)$ \\
\hline PBI 3 & $\mathrm{n}=20(34 \%)$ \\
\hline PBI 4 & $n=6(10 \%)$ \\
\hline \multicolumn{2}{|c|}{ parodontaler Befund (maximaler PSI) } \\
\hline PSI 0 & $\mathrm{n}=3(5 \%)$ \\
\hline PSI 1-2 & $n=31(53 \%)$ \\
\hline PSI 3 & $\mathrm{n}=23(39 \%)$ \\
\hline PSI 4 & $\mathrm{n}=0(0 \%)$ \\
\hline $\mathrm{SA}=$ Standardabweichung & \\
\hline
\end{tabular}

Die Abbildung 6 zeigt die PBI-Werte aller durchgeführten Messungen. In Abbildung 7 sind alle PSI-Werte der einzelnen Sextanten im Überblick dargestellt.

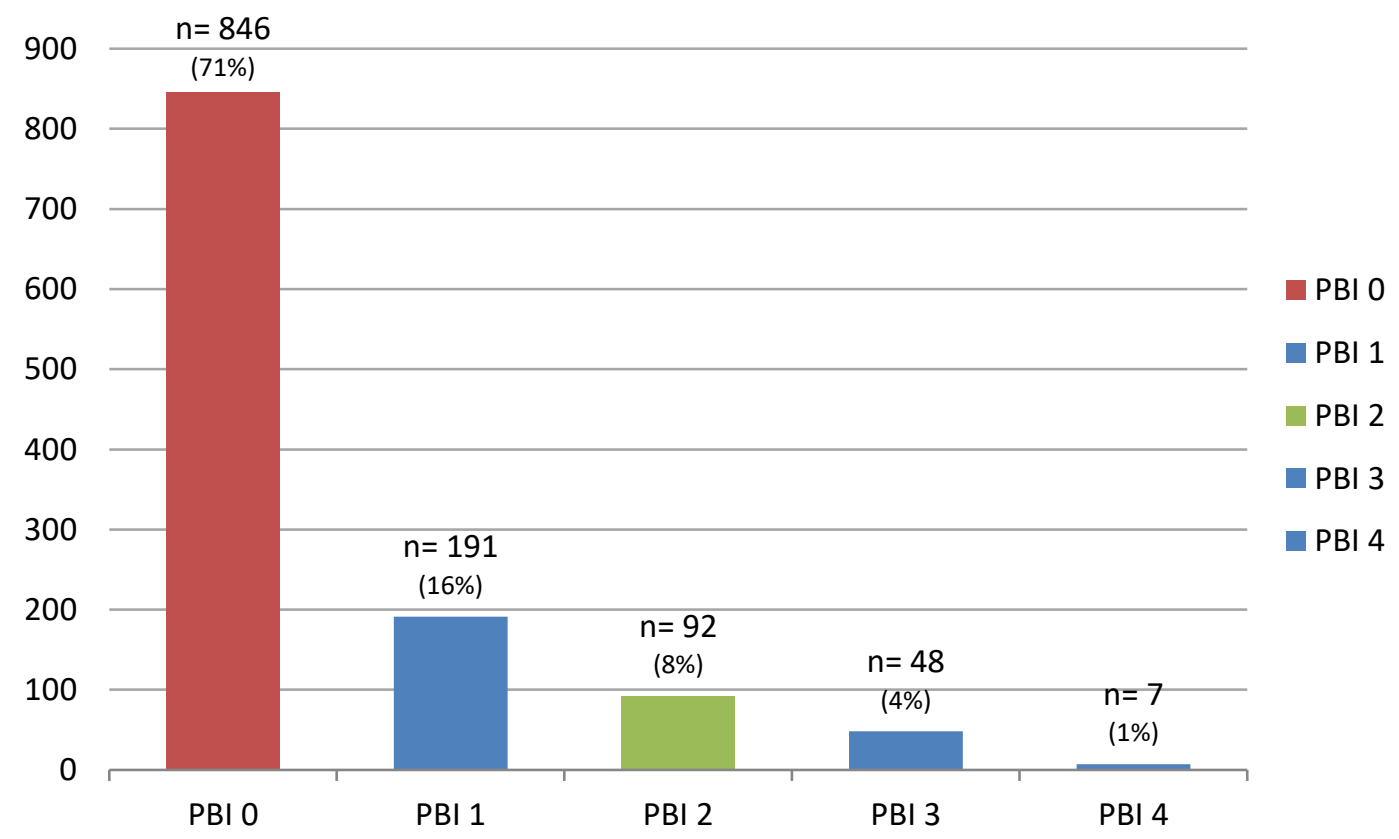

Abb. 6: Verteilung der PBI-Werte aller Messstellen absolut und in \% 


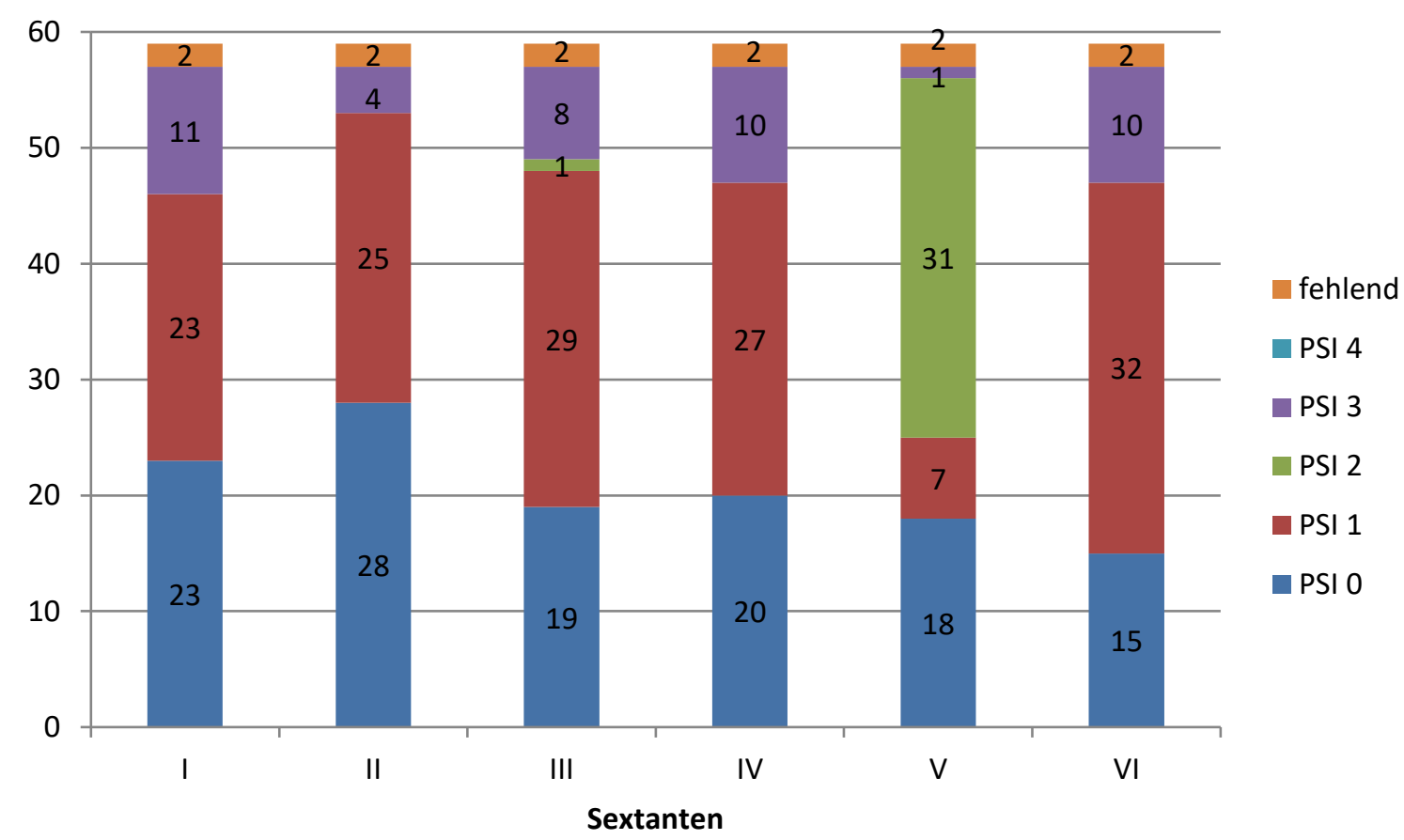

Abb. 7: Graphische Darstellung der Verteilung der PSI-Werte (absolut) in den einzelnen Sextanten

\subsubsection{Kiefergelenksdiagnostik bei Kindern mit juveniler Arthritis}

In Bezug auf die Parameter Kiefergelenkgeräusche, Unterkieferblockade und Schmerzen waren 24 der 59 Patienten (41\%) anamnestisch und 32 Patienten (54\%) klinisch auffällig. Bei der Anamnese waren die Angabe von Schmerzen $(n=17 / 29 \%)$, bei der klinischen Diagnostik hingegen das Kiefergelenkgeräusch ( $n=14 / 34 \%)$ die am häufigsten auftretenden Merkmale.

Bei acht Patienten waren im Röntgenbild entweder die zu beurteilenden Strukturen mindestens eines Gelenkes nicht abgebildet, oder es lagen Anomalien der Gelenkstrukturen vor, welche nicht mit den Definitionen der Röntgenscores zu vereinbaren waren. Diese Patienten wurden nicht in die Auswertung zu Kiefergelenkanomalien mit einbezogen. 52\% der Patienten ( $n=27)$ wiesen im Röntgenbild keinerlei Anomalien der Kiefergelenkköpfchen auf, bei 29\% war der maximale Score von 1 festzustellen. 18\% der Patienten zeigten deutlich erkennbare Strukturveränderungen entsprechend Score 2 oder 3 (Tab. 10). 
Tab. 10: Anamnestische, klinische und röntgenologische Parameter der Kiefergelenksdiagnostik

\begin{tabular}{ll}
\hline anamnestische Auffälligkeiten & Anzahl der Patienten (\%) \\
\hline auffällig gesamt & $n=24(41 \%)$ \\
Kiefergelenkgeräusche & $n=14(24 \%)$ \\
Blockade & $n=9(15 \%)$ \\
Schmerzen & $n=17(29 \%)$ \\
\hline klinische Befunde & Anzahl der Patienten (\%) \\
\hline auffällig gesamt & $n=32(54 \%)$ \\
Kiefergelenkgeräusche & $n=20(34 \%)$ \\
Blockade & $n=11(19 \%)$ \\
Schmerzen bei Muskelpalpation & $n=4(7 \%)$ \\
Schmerzen bei Gelenkpalpation & $n=10(17 \%)$ \\
\hline Röntgenbefund (Scoring) & Anzahl der Patienten (\%) \\
\hline Score 0 & $n=27(53 \%)$ \\
Score 1 & $n=15(29 \%)$ \\
Score 2 & $n=5(10 \%)$ \\
Score 3 & $n=4(8 \%)$
\end{tabular}

Abbildung 8 zeigt zusätzlich die Scores aller beurteilbaren Gelenke rechts- und linksseitig.

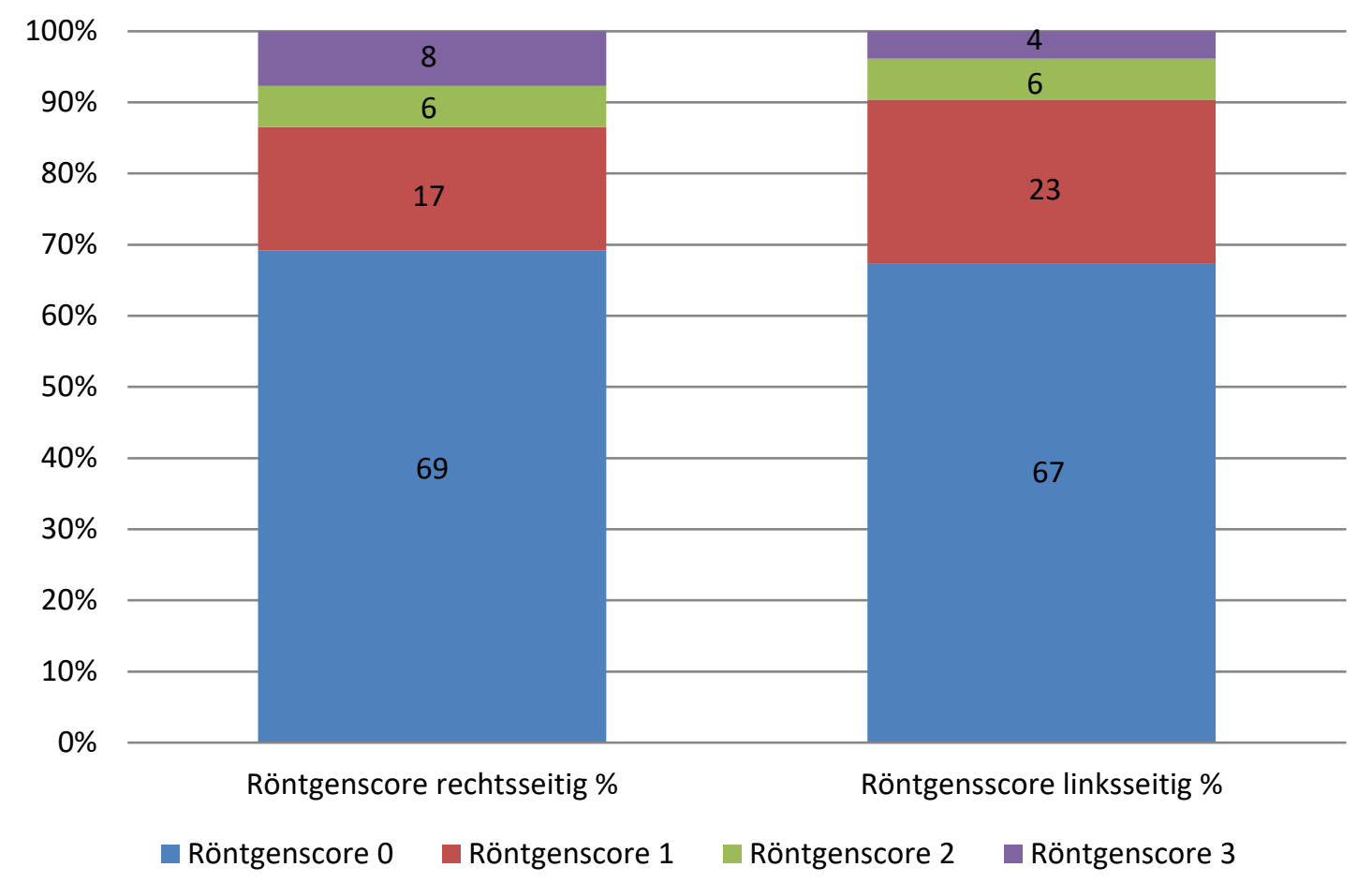

Abb. 8: Röntgenscore aller Kiefergelenke links- und rechtsseitig in \% 


\subsection{Untersuchungen zum Zusammenhang zwischen oraler Ge- sundheit und Kiefergelenksbefunden}

Patienten mit normaler oder eingeschränkter MÖ wiesen keine signifikanten Unterschiede in der dentalen Gesundheit auf, obgleich insbesondere bei Betrachtung des mittleren dmf-t/DMF-T höhere Werte beim Vorliegen einer eingeschränkten MÖ ermittelt wurden (Tab. 11). Tendenziell konnte dies auch bei der gingivalen Entzündung und parodontalen Befunden beobachtet werden (Tab. 11, 12 und 13). Tabelle 12 und Tabelle 13 zeigen zusätzlich, dass der Patientenanteil mit einer kiefergelenkbezogenen Funktionseinschränkung mit zunehmenden PSI-Werten anstieg. Bei den Untersuchungen konnten durch den Exakten Test nach Fisher jedoch keine signifikanten Zusammenhänge der PSI-Werte zur eingeschränkten MÖ in klinischer $(\mathrm{Cl}=0,205 ; \mathrm{p}=0,519)$ oder anamnestischer $(\mathrm{Cl}=0,128 ; \mathrm{p}=0,942)$ Diagnostik ermittelt werden.

Tab. 11: Unterschiede bzgl. oraler Erkrankungen bei JIA-Patienten mit und ohne eingeschränkte MÖ

\begin{tabular}{|c|c|c|c|c|c|c|}
\hline & Mundöffnung & $\mathbf{N}$ & $\mathrm{MW} \pm \mathrm{SA}$ & $\begin{array}{c}\text { mittlere } \\
\text { Rangsumme }\end{array}$ & Z-Wert & $p$-Wert \\
\hline \multirow{4}{*}{ 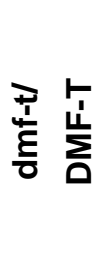 } & MÖ $\downarrow$ in klin. Diagnostik & 15 & $3,5 \pm 4,5$ & 30,8 & \multirow{2}{*}{$-0,222$} & \multirow{2}{*}{0,824} \\
\hline & normal in klin. Diagnostik & 44 & $2,3 \pm 2,2$ & 29,7 & & \\
\hline & MÖ $\downarrow$ in Anamnese & 9 & $3,6 \pm 5,2$ & 28,9 & \multirow{2}{*}{$-0,215$} & \multirow{2}{*}{0,830} \\
\hline & normal in Anamnese & 50 & $2,4 \pm 2,4$ & 30,2 & & \\
\hline \multirow{4}{*}{ 占 } & MÖ $\downarrow$ in klin. Diagnostik & 15 & $0,7 \pm 1,5$ & 27,7 & \multirow{2}{*}{$-0,713$} & \multirow{2}{*}{0,476} \\
\hline & normal in klin. Diagnostik & 44 & $0,9 \pm 1,5$ & 30,8 & & \\
\hline & MÖ $\downarrow$ in Anamnese & 9 & $0,9 \pm 1,8$ & 27,4 & \multirow{2}{*}{$-0,580$} & \multirow{2}{*}{0,562} \\
\hline & normal in Anamnese & 50 & $0,9 \pm 1,4$ & 30,5 & & \\
\hline \multirow{4}{*}{$\begin{array}{l}\overline{\mathbf{m}} \\
\underline{a}\end{array}$} & MÖ $\downarrow$ in klin. Diagnostik & 15 & $0,5 \pm 0,3$ & 31,0 & \multirow{2}{*}{$-0,533$} & \multirow{2}{*}{0,580} \\
\hline & normal in klin. Diagnostik & 42 & $0,5 \pm 0,3$ & 28,3 & & \\
\hline & MÖ $\downarrow$ in Anamnese & 9 & $0,5 \pm 0,3$ & 35,4 & \multirow{2}{*}{$-1,259$} & \multirow{2}{*}{0,208} \\
\hline & normal in Anamnese & 48 & $0,4 \pm 0,3$ & 27,8 & & \\
\hline
\end{tabular}

MÖ $\downarrow$ = eingeschränkte MÖ; MW = Mittelwert; SA = Standardabweichung, statistischer Test = Mann-Whitney-U 
Tab. 12: Kreuztabelle zum Zusammenhang der klinisch diagnostizierten MÖ mit dem PSI-Maximum

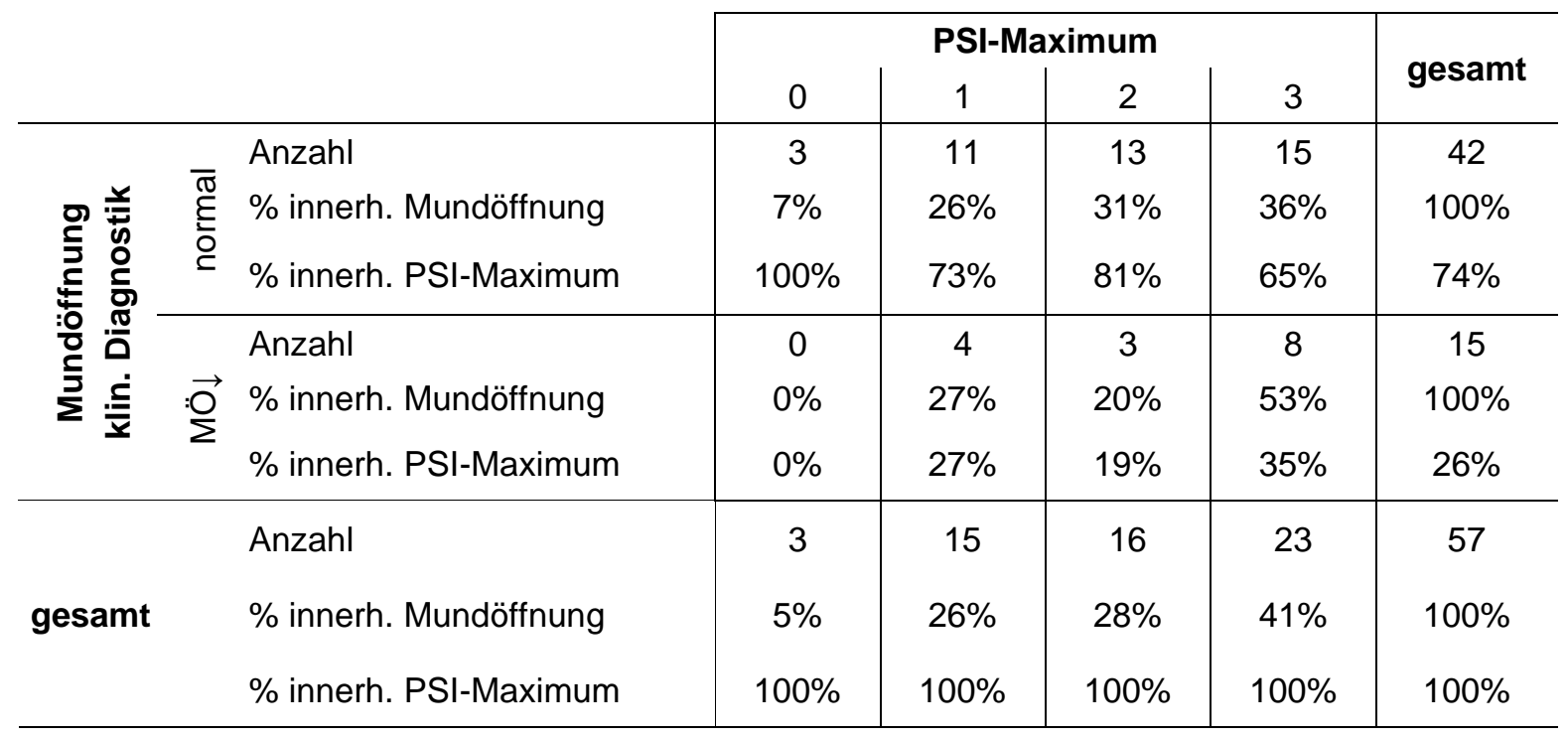

MÖ $\downarrow$ = eingeschränkte Mundöffnung

Tab. 13: Kreuztabelle zum Zusammenhang der anamnestisch diagnostizierten MÖ mit dem PSIMaximum

\begin{tabular}{|c|c|c|c|c|c|c|c|}
\hline & \multicolumn{4}{|c|}{ PSI-Maximum } & \multirow{2}{*}{ gesam } \\
\hline & & & 0 & 1 & 2 & 3 & \\
\hline \multirow{2}{*}{ 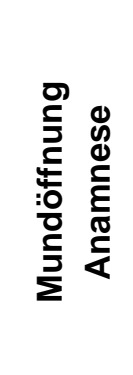 } & 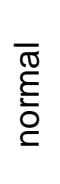 & $\begin{array}{l}\text { Anzahl } \\
\% \text { innerh. Mundöffnung } \\
\% \text { innerh. PSI-Maximum }\end{array}$ & $\begin{array}{c}3 \\
6 \% \\
100 \%\end{array}$ & $\begin{array}{c}12 \\
25 \% \\
80 \%\end{array}$ & $\begin{array}{c}14 \\
29 \% \\
88 \%\end{array}$ & $\begin{array}{c}19 \\
40 \% \\
83 \%\end{array}$ & $\begin{array}{c}48 \\
100 \% \\
84 \%\end{array}$ \\
\hline & $: \vec{o}$ & $\begin{array}{l}\text { Anzahl } \\
\% \text { innerh. Mundöffnung } \\
\% \text { innerh. PSI-Maximum }\end{array}$ & $\begin{array}{c}0 \\
0 \% \\
0 \%\end{array}$ & $\begin{array}{c}3 \\
33 \% \\
20 \%\end{array}$ & $\begin{array}{c}2 \\
22 \% \\
12 \%\end{array}$ & $\begin{array}{c}4 \\
45 \% \\
17 \%\end{array}$ & $\begin{array}{c}9 \\
100 \% \\
16 \%\end{array}$ \\
\hline gesamt & & $\begin{array}{l}\text { Anzahl } \\
\% \text { innerh. Mundöffnung } \\
\% \text { innerh. PSI-Maximum }\end{array}$ & $\begin{array}{c}3 \\
5 \% \\
100 \%\end{array}$ & $\begin{array}{c}15 \\
26 \% \\
100 \%\end{array}$ & $\begin{array}{c}16 \\
28 \% \\
100 \%\end{array}$ & $\begin{array}{c}23 \\
41 \% \\
100 \%\end{array}$ & $\begin{array}{c}57 \\
100 \% \\
100 \%\end{array}$ \\
\hline
\end{tabular}

$M O ̈ \downarrow=$ eingeschränkte Mundöffnung

Die Analyseergebnisse zu möglichen Zusammenhängen zwischen klinischer bzw. anamnestischer Schmerzdiagnostik und oralen Gesundheitsparametern sind in Tab. 14 dargestellt. Auffällig waren hier die deutlich höheren Mittelwerte und Rangsummen in allen Bereichen der oralen Gesundheit (d-t/D-T, dmf-T/DMF-T und $\mathrm{PBI}$ ) bei Patienten mit positiver klinischer Schmerzdiagnostik. Signifikante Unterschiede konnten jedoch nicht festgestellt werden. Gleiches gilt für die in Tabelle 15 und 16 dargestellten Ergebnisse zum Zusammenhang des PSI zu diagnostizierten Kiefergelenkschmerzen innerhalb der klinischen $(\mathrm{Cl}=0,158, \mathrm{p}$-Wert $=0,852)$ 
oder anamnestischen $(\mathrm{Cl}=0,71, \mathrm{p}$-Wert=0,969) Diagnostik. Sowohl bei niedrigen als auch bei hohen PSI-Max-Werten überwog der Anteil der schmerzfreien Patienten deutlich.

Tab. 14: Unterschiede bzgl. oraler Erkrankungen bei JIA-Patienten mit und ohne Schmerzen

\begin{tabular}{|c|c|c|c|c|c|c|}
\hline & Schmerzen im Kiefergelenk & $\mathbf{N}$ & $M W \pm S A$ & $\begin{array}{c}\text { mittlere } \\
\text { Rangsumme }\end{array}$ & Z-Wert & $\begin{array}{c}\text { p- } \\
\text { Wert }\end{array}$ \\
\hline \multirow{4}{*}{ 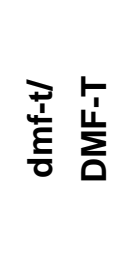 } & positiv in Anamnese & 17 & $3,1 \pm 4,1$ & 30,56 & \multirow{2}{*}{$-0,162$} & \multirow{2}{*}{0,871} \\
\hline & ohne Befund in Anamnese & 42 & $2,3 \pm 2,4$ & 29,77 & & \\
\hline & positiv in klin. Diagnostik & 12 & $3.9 \pm 4,4$ & 35,04 & \multirow{2}{*}{$-1,163$} & \multirow{2}{*}{0,245} \\
\hline & ohne Befund in klin. Diagnostik & 47 & $2,2 \pm 2,4$ & 28,71 & & \\
\hline \multirow{4}{*}{ ti } & positiv in Anamnese & 17 & $1,1 \pm 1,7$ & 29,65 & \multirow{2}{*}{$-0,118$} & \multirow{2}{*}{0,906} \\
\hline & ohne Befund in Anamnese & 42 & $0,8 \pm 1,4$ & 30,14 & & \\
\hline & positiv in klin. Diagnostik & 12 & $1,6 \pm 1,9$ & 35,79 & \multirow{2}{*}{$-1,532$} & \multirow{2}{*}{0,125} \\
\hline & ohne Befund in klin. Diagnostik & 47 & $0,7 \pm 1,3$ & 28,52 & & \\
\hline \multirow{4}{*}{$\overline{\mathbf{m}}$} & positiv in Anamnese & 17 & $0,4 \pm 0,2$ & 27,62 & \multirow{2}{*}{$-0,410$} & \multirow{2}{*}{0,682} \\
\hline & ohne Befund in Anamnese & 40 & $0,5 \pm 0,3$ & 29,59 & & \\
\hline & positiv in klin. Diagnostik & 12 & $0,6 \pm 0,4$ & 34,25 & \multirow{2}{*}{$-1,234$} & \multirow{2}{*}{0,217} \\
\hline & ohne Befund in klin. Diagnostik & 45 & $0,4 \pm 0,3$ & 27,60 & & \\
\hline
\end{tabular}

Tab. 15: Kreuztabelle zum Zusammenhang klinisch diagnostizierter Schmerzen mit dem PSI-Maximum

\begin{tabular}{|c|c|c|c|c|c|c|}
\hline & \multicolumn{4}{|c|}{ PSI-Maximum } & \multirow{2}{*}{ gesamt } \\
\hline & & 0 & 1 & 2 & 3 & \\
\hline \multirow{6}{*}{ 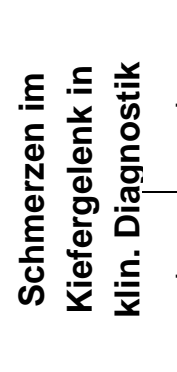 } & Anzahl & 0 & 4 & 4 & 4 & 12 \\
\hline & \% innerh. Schmerzen & $0 \%$ & $33,3 \%$ & $33,3 \%$ & $33,3 \%$ & $100 \%$ \\
\hline & $\%$ innerh. PSI-Maximum & $0 \%$ & $27 \%$ & $25 \%$ & $17 \%$ & $21,1 \%$ \\
\hline & Anzahl & 3 & 11 & 12 & 19 & 45 \\
\hline & \% innerh. Schmerzen & $7 \%$ & $24 \%$ & $27 \%$ & $42 \%$ & $100 \%$ \\
\hline & $\%$ innerh. PSI-Maximum & $100 \%$ & $74 \%$ & $75 \%$ & $83 \%$ & $78,9 \%$ \\
\hline \multirow{3}{*}{ gesamt } & Anzahl & 3 & 15 & 16 & 23 & 57 \\
\hline & $\%$ innerh. Schmerzen & $5 \%$ & $26 \%$ & $28 \%$ & $41 \%$ & $100 \%$ \\
\hline & $\%$ innerh. PSI-Maximum & $100 \%$ & $100 \%$ & $100 \%$ & $100 \%$ & $100 \%$ \\
\hline
\end{tabular}


Tab. 16: Kreuztabelle zum Zusammenhang anamnestisch diagnostizierter Schmerzen mit dem PSIMaximum

\begin{tabular}{|c|c|c|c|c|c|c|c|}
\hline & \multicolumn{4}{|c|}{ PSI-Maximum } & \multirow{2}{*}{ gesamt } \\
\hline & & & 0 & 1 & 2 & 3 & \\
\hline \multirow{6}{*}{ 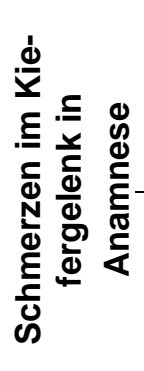 } & \multirow{3}{*}{.$\underline{\widetilde{\sigma}}$} & Anzahl & 1 & 5 & 4 & 7 & 17 \\
\hline & & $\%$ innerh. Schmerzen & $6 \%$ & $29 \%$ & $24 \%$ & $41 \%$ & $100 \%$ \\
\hline & & $\%$ innerh. PSI-Maximum & $33 \%$ & $33 \%$ & $25 \%$ & $30 \%$ & $29,8 \%$ \\
\hline & \multirow{3}{*}{ 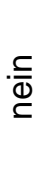 } & Anzahl & 2 & 10 & 12 & 16 & 40 \\
\hline & & $\%$ innerh. Schmerzen & $5 \%$ & $25 \%$ & $30 \%$ & $40 \%$ & $100 \%$ \\
\hline & & $\%$ innerh. PSI-Maximum & $67 \%$ & $67 \%$ & $75 \%$ & $70 \%$ & $70,2 \%$ \\
\hline \multirow{3}{*}{ gesamt } & & Anzahl & 3 & 15 & 16 & 23 & 57 \\
\hline & & $\%$ innerh. Schmerzen & $5 \%$ & $26 \%$ & $28 \%$ & $41 \%$ & $100 \%$ \\
\hline & & $\%$ innerh. PSI-Maximum & $100 \%$ & $100 \%$ & $100 \%$ & $100 \%$ & $100 \%$ \\
\hline
\end{tabular}

In Hinblick auf die röntgenologischen Kiefergelenksbefunde unterschieden sich Patienten mit auffälligem Röntgenscore (RS 1-3) von Patienten mit RS 0 nur geringfügig und nicht signifikant in den oralen Mundgesundheitsparametern dmft/DMF-T, d-t/D-T und PBI (Tab. 17). In Tabelle 18 ist auffällig, dass die Hälfte der Patienten mit röntgenologischen Kiefergelenksauffälligkeiten den höchsten PSIMax-Wert von 3 aufwiesen. Ein signifikanter Zusammenhang zwischen den Röntgenbefunden und dem PSI konnte allerdings ebenfalls nicht ermittelt werden $(\mathrm{Cl}=0,23, \mathrm{p}-$ Wert $=0,514)(\mathrm{Tab} .18)$.

Tab. 17: Unterschiede bzgl. oraler Erkrankungen bei JIA-Patienten mit und ohne röntgenologische Auffälligkeiten

\begin{tabular}{|c|c|c|c|c|c|c|}
\hline & Röntgenscore & $\mathbf{N}$ & $M W \pm S A$ & $\begin{array}{c}\text { mittlere } \\
\text { Rangsumme }\end{array}$ & Z-Wert & $\begin{array}{c}\text { p- } \\
\text { Wert }\end{array}$ \\
\hline 突 忘 & $\begin{array}{l}\text { positiv in klin. Diagnostik (RS 1-3) } \\
\text { ohne Befund (RS 0) }\end{array}$ & $\begin{array}{l}24 \\
27\end{array}$ & $\begin{array}{l}3,0 \pm 3,7 \\
2,3 \pm 2,3\end{array}$ & $\begin{array}{l}26,44 \\
25,61\end{array}$ & $-0,202$ & 0,840 \\
\hline$\stackrel{\vdash}{\circ}$ & $\begin{array}{l}\text { positiv in klin. Diagnostik (RS 1-3) } \\
\text { ohne Befund (RS 0) }\end{array}$ & $\begin{array}{l}24 \\
27\end{array}$ & $\begin{array}{l}0,9 \pm 1,5 \\
1,0 \pm 1,6\end{array}$ & $\begin{array}{l}25,88 \\
26,11\end{array}$ & $-0,066$ & 0,947 \\
\hline $\begin{array}{l}\overline{\mathbf{g}} \\
\underline{Q}\end{array}$ & $\begin{array}{l}\text { positiv in klin. Diagnostik (RS 1-3) } \\
\text { ohne Befund (RS 0) }\end{array}$ & $\begin{array}{l}23 \\
26\end{array}$ & $\begin{array}{l}0,5 \pm 0,3 \\
0,4 \pm 0,3\end{array}$ & $\begin{array}{l}25,74 \\
24,35\end{array}$ & $-0,341$ & 0,733 \\
\hline
\end{tabular}


Tab. 18: Kreuztabelle zum Zusammenhang röntgenologischer Kiefergelenksauffälligkeiten mit dem PSIMaximum

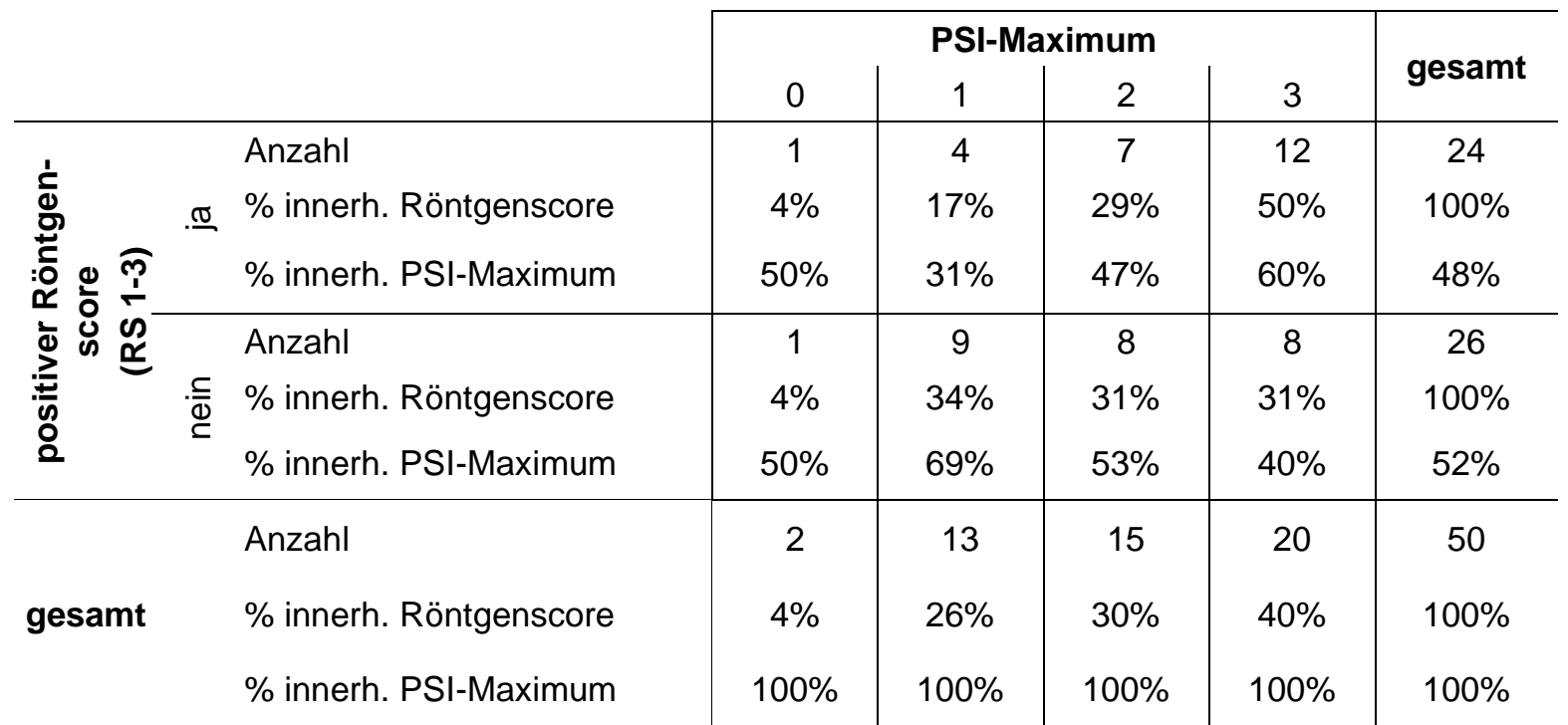

RS = Röntgenscore

\subsection{Orale Gesundheit und Kiefergelenksbefunde in Abhängigkeit von Erkrankungsform, Erkrankungsdauer und Blutparame- tern}

\subsubsection{Vergleich von Parametern der oralen Gesundheit und röntgenologi- scher Kiefergelenksbefunde in Abhängigkeit von der Arthritisform}

Für die Parameter dmf-t/DMF-T, d-t/D-T und PBI konnten keine signifikanten Unterschiede zwischen Oligoarthritis- und Polyarthritispatienten festgestellt werden (Tab. 19).

Tabelle 20 zeigt die maximalen PSI-Werte (PSI-Max) für OA- und PA-Patienten. Auffallend ist, dass in der Gruppe der PA-Patienten keine Person einen PSI-Max von 0 aufwies, jedoch 50\% der Patienten dieser Gruppe den PSI-Max von 3. Im Gegensatz dazu verteilten sich die OA-Patienten gleichmäßiger auf die einzelnen PSI-Grade. Der Anteil der Patienten mit einem PSI-Max von 3 war um 16Prozentpunkte geringer als in der Gruppe der PA-Patienten. Ein möglicher Zusammenhang zwischen dem PSI-Maximum und einer Arthritisform konnte durch den Exakten Test nach Fisher statistisch jedoch nicht festgestellt werden $(\mathrm{Cl}=0,233 ; \mathrm{p}=0,452)$. 
Tab. 19: Tabellarische Darstellung der Unterschiede im Vorkommen oraler Erkrankungen bei Oligobzw. Polyarthritis

\begin{tabular}{|c|c|c|c|c|c|c|}
\hline & \multirow[b]{2}{*}{$\mathbf{N}$} & \multirow[b]{2}{*}{$M W \pm S A$} & \multirow[b]{2}{*}{$\begin{array}{c}\text { mittlerer } \\
\text { Rang }\end{array}$} & \multirow[b]{2}{*}{ Z-Wert } & \multirow[b]{2}{*}{$p$-Wert } \\
\hline & & & & & & \\
\hline \multirow{3}{*}{$\begin{array}{l}\text { Kariesindex } \\
\text { dmf-T/DMF-T }\end{array}$} & Oligoarthritis & 37 & $2,7 \pm 3,2$ & 30,6 & & \\
\hline & Polyarthritis & 22 & $2,3 \pm 2,6$ & 29,0 & 0,360 & 0,719 \\
\hline & gesamt & 59 & $2,6 \pm 3,0$ & & & \\
\hline \multirow{3}{*}{$\begin{array}{l}\text { Kariesindex } \\
\text { dt/DT }\end{array}$} & Oligoarthritis & 37 & $0,8 \pm 1,5$ & 29,0 & & \\
\hline & Polyarthritis & 22 & $1,0 \pm 1,5$ & 31,6 & 0,642 & 0,521 \\
\hline & gesamt & 59 & $0,9 \pm 1,5$ & & & \\
\hline \multirow{3}{*}{ PBI } & Oligoarthritis & 35 & $0,5 \pm 0,3$ & 29,5 & & \\
\hline & Polyarthritis & 22 & $0,4 \pm 0,3$ & 28,1 & 0,312 & 0,755 \\
\hline & gesamt & 57 & $0,5 \pm 0,3$ & & & \\
\hline
\end{tabular}

MW = Mittelwert; SA = Standardabweichung; statistischer Test = Mann-Whitney-U

Tab. 20: Kreuztabelle zur Darstellung des Zusammenhangs zwischen dem PSI-Maximum und der Oligobzw. Polyarthritis

\begin{tabular}{ll|c|c|c|c|c}
\cline { 3 - 6 } & & \multicolumn{3}{c|}{ PSI-Maximum } & \multirow{2}{*}{ gesamt } \\
\hline \multirow{3}{*}{ Oligoarthritis } & Anzahl & 0 & 1 & 2 & 3 & \\
& $\%$ innerhalb Oligoarthritis & 3 & 9 & 11 & 12 & 35 \\
& $\%$ innerhalb PSI-Maximum & $9 \%$ & $26 \%$ & $31 \%$ & $34 \%$ & $100 \%$ \\
\hline \multirow{3}{*}{ Polyarthritis } & \% innerhalb Polyarthritis & 0 & 6 & 5 & 11 & 22 \\
& Anzahl & $0 \%$ & $27 \%$ & $23 \%$ & $50 \%$ & $100 \%$ \\
\hline \multirow{2}{*}{ gesamnerhalb PSI-Maximum } & $0 \%$ & $40 \%$ & $31 \%$ & $48 \%$ & $39 \%$ \\
\cline { 2 - 7 } & Anzahl & 3 & 15 & 16 & 23 & 57 \\
& $\%$ innerhalb Oligo-/Polyarthritis & $5 \%$ & $26 \%$ & $28 \%$ & $40 \%$ & $100 \%$ \\
& $\%$ innerhalb PSI-Maximum & $100 \%$ & $100 \%$ & $100 \%$ & $100 \%$ & $100 \%$
\end{tabular}

Hinsichtlich der Röntgenscores (RS) war bei den RS-Werten von 0-2 ein deutlich größerer Anteil bei OA-Patienten gegenüber PA-Patienten zu erkennen. Bei RS 3 überwog der Anteil der PA-Patienten mit 75\%. Ein signifikanter Zusammenhang zum RS konnte für OA- bzw. PA-Patienten allerdings nicht festgestellt werden (Exakter Test nach Fisher: $\mathrm{Cl}=0,256, \mathrm{p}=0,406)$ (Tab.21). 
Tab. 21: Kreuztabelle zur Darstellung des Zusammenhangs zwischen dem Röntgenscore und der Oligobzw. Polyarthritis

\begin{tabular}{|c|c|c|c|c|c|c|}
\hline & \multirow{2}{*}{\multicolumn{4}{|c|}{ Röntgenscore }} & \multirow{3}{*}{ gesamt } \\
\hline & & & & & & \\
\hline & & 0 & 1 & 2 & 3 & \\
\hline \multirow{3}{*}{ Oligoarthritis } & Anzahl & 18 & 11 & 3 & 1 & 33 \\
\hline & $\%$ innerh. von Oligoarthritis & $55 \%$ & $33 \%$ & $9 \%$ & $3 \%$ & $100 \%$ \\
\hline & \% innerh. von Röntgenscore & $67 \%$ & $73 \%$ & $60 \%$ & $25 \%$ & $65 \%$ \\
\hline \multirow{3}{*}{ Polyarthritis } & Anzahl & 9 & 4 & 2 & 3 & 18 \\
\hline & $\%$ innerh. von Polyarthritis & $50 \%$ & $22 \%$ & $11 \%$ & $17 \%$ & $100 \%$ \\
\hline & \% innerh. von Röntgenscore & $33 \%$ & $27 \%$ & $40 \%$ & $75 \%$ & $35 \%$ \\
\hline \multirow{3}{*}{ gesamt } & Anzahl & 27 & 15 & 5 & 4 & 51 \\
\hline & $\%$ innerh. Oligo-/Polyarthritis & $53 \%$ & $29 \%$ & $10 \%$ & $8 \%$ & $100 \%$ \\
\hline & \% innerh. von Röntgenscore & $100 \%$ & $100 \%$ & $100 \%$ & $100 \%$ & $100 \%$ \\
\hline
\end{tabular}

\subsubsection{Vergleich von Parametern der oralen Gesundheit in Abhängigkeit von Funktionseinschränkungen bei Mitbeteiligung oberer Gelenke}

Zwischen den Patienten mit und ohne Beteiligung eines oder mehrerer der oberen Gelenke gab es keine signifikanten Unterschiede beim mittleren dmf-t/DMF-T $(2,6 \pm 2,8$ bzw. 2,6 $\pm 3,2)$ und dem d-t/D-T $(0,9 \pm 0,5$ bzw. 0,9 $\pm 1,6)$ (Tab. 22). Auch bei der Analyse der Häufigkeitsunterschiede des dmf-t/DMF-T und des d-t/D-T, bezogen auf die einzelnen Gelenkbereiche, zeigten sich zwischen den Patientengruppen nur minimale Abweichungen in den Mittelwerten und den mittleren Rangsummen (Tab. 22). Lediglich in Bezug auf die Schultergelenke waren sowohl für den mittleren dmf-t/DMF-T als auch für den mittleren d-t/D-T deutliche Unterschiede zwischen Patienten mit einer Beteiligung $(6,0 \pm 4,4$ bzw. 0,9 $\pm 0,5)$ und Patienten ohne Beteiligung $(2,4 \pm 2,8$ bzw. $0,8 \pm 1,4)$ zu erkennen. Ein signifikanter Unterschied konnte jedoch nicht festgestellt werden ( $p=0,81$ bzw. $p=0,152)$.

Ebenso waren für keine der überprüften Gelenkformen signifikante Unterschiede einzelner mittlerer PBI-Werte und Rangsummen zu ermitteln. Es ist insgesamt auffallend, dass Patienten ohne eine Beteiligung der oberen Gelenke tendenziell sogar höhere PBI-Werte aufwiesen als Patienten mit einer Beteiligung (Tab. 22). 
Tab. 22: Unterschiede bzgl. oraler Erkrankungen bei JIA-Patienten mit und ohne Erkrankung der oberen Extremitäten

\begin{tabular}{|c|c|c|c|c|c|c|}
\hline & Gelenkmitbeteiligung & $\mathbf{N}$ & $\mathrm{MW} \pm \mathrm{SA}$ & $\begin{array}{c}\text { mittlere } \\
\text { Rangsumme }\end{array}$ & Z-Wert & p-Wert \\
\hline \multirow{10}{*}{ 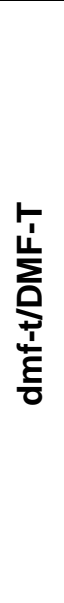 } & Ob. Extremitäten ja & 30 & $2,6 \pm 2,8$ & 30,4 & \multirow{2}{*}{,- 201} & \multirow{2}{*}{,840 } \\
\hline & Ob. Extremitäten nein & 29 & $2,6 \pm 3,2$ & 29,6 & & \\
\hline & Finger ja & 21 & $2,3 \pm 2,5$ & 29,5 & \multirow{2}{*}{,- 186} & \multirow{2}{*}{,853 } \\
\hline & Finger nein & 38 & $2,7 \pm 3,2$ & 30,3 & & \\
\hline & Handgelenk ja & 19 & $2,3 \pm 2,8$ & 28,5 & \multirow{2}{*}{,- 472} & \multirow{2}{*}{,637 } \\
\hline & Handgelenk nein & 40 & $2,7 \pm 3,1$ & 30,7 & & \\
\hline & Ellenbogen ja & 15 & $2,7 \pm 2,9$ & 31,6 & \multirow{2}{*}{,- 427} & \multirow{2}{*}{, 670} \\
\hline & Ellenbogen nein & 44 & $2,5 \pm 3,0$ & 29,5 & & \\
\hline & Schulter ja & 3 & $6,0 \pm 4,4$ & 46,5 & 174 & \\
\hline & Schulter nein & 56 & $2,4 \pm 2,8$ & 29,1 & $-1,144$ & , \\
\hline \multirow{10}{*}{ 它 } & Ob. Extremitäten ja & 30 & $0,9 \pm 0,5$ & 30,4 & \multirow{2}{*}{,- 186} & \multirow{2}{*}{,852 } \\
\hline & Ob. Extremitäten nein & 29 & $0,9 \pm 1,6$ & 29,6 & & \\
\hline & Finger ja & 21 & $0,7 \pm 1,1$ & 28,8 & \multirow{2}{*}{,- 473} & \multirow{2}{*}{,636 } \\
\hline & Finger nein & 38 & $1,0 \pm 1,7$ & 30,7 & & \\
\hline & Handgelenk ja & 19 & $1,1 \pm 1,6$ & 32,2 & \multirow{2}{*}{,- 798} & \multirow{2}{*}{,425 } \\
\hline & Handgelenk nein & 40 & $0,8 \pm 1,4$ & 29,0 & & \\
\hline & Ellenbogen ja & 15 & $1,1 \pm 1,5$ & 32,9 & \multirow{2}{*}{,- 886} & \multirow{2}{*}{,375 } \\
\hline & Ellenbogen nein & 44 & $0,8 \pm 1,5$ & 29,0 & & \\
\hline & Schulter ja & 3 & $2,3 \pm 2,5$ & 41,9 & \multirow{2}{*}{$-1,434$} & \multirow{2}{*}{,152 } \\
\hline & Schulter nein & 56 & $0,8 \pm 1,4$ & 29,4 & & \\
\hline \multirow{10}{*}{$\overline{\mathbf{m}}$} & Ob. Extremitäten ja & 29 & $0,5 \pm 0,3$ & 29,4 & \multirow{2}{*}{,- 192} & \multirow{2}{*}{,848 } \\
\hline & Ob. Extremitäten nein & 28 & $0,5 \pm 0,4$ & 28,6 & & \\
\hline & Finger ja & 21 & $0,4 \pm 0,3$ & 27,4 & \multirow{2}{*}{,- 563} & \multirow{2}{*}{, 574} \\
\hline & Finger nein & 36 & $0,5 \pm 0,4$ & 29,9 & & \\
\hline & Handgelenk ja & 19 & $0,4 \pm 0,3$ & 27,8 & \multirow{2}{*}{,- 398} & \multirow{2}{*}{,691 } \\
\hline & Handgelenk nein & 38 & $0,5 \pm 0,3$ & 29,3 & & \\
\hline & Ellenbogen ja & 13 & $0,4 \pm 0,3$ & 25,0 & คి & 010 \\
\hline & Ellenbogen nein & 44 & $0,5 \pm 0,3$ & 30,2 &,- 999 & , \\
\hline & Schulter ja & 3 & $0,5 \pm 0,3$ & 29,8 & ח०० & מ२० \\
\hline & Schulter nein & 54 & $0,5 \pm 0,3$ & 29,0 & בס,-', &, $9<9$ \\
\hline
\end{tabular}

MW = Mittelwerte SA = Standardabweichung; Ob. Extremitäten = obere Extremtäten; statistischer Test $=$ Mann-Whitney-U

Tabelle 23 ist zu entnehmen, dass der Anteil der Patienten sowohl ohne als auch mit einer Gelenkbeteiligung mit zunehmenden PSI-Max-Werten ansteigt. Auffällig ist jedoch, dass der Patientenanteil mit einer Beteiligung der oberen Gelenke im 
Vergleich zur Gruppe ohne Gelenkbeteiligung ausschließlich in der Patientengruppe mit dem höchsten gemessenen PSI von 3 überwog. Zwischen den Variablen „Mitbeteiligung der Gelenke“ und „PSI-Max“ konnte allerdings kein signifikanter Zusammenhang festgestellt werden $(\mathrm{Cl}=0,134, \mathrm{p}=0,795)$.

Tab. 23: Kreuztabelle zum Zusammenhang der Mitbeteiligung oberer Gelenke und dem PSI-Maximum

\begin{tabular}{|c|c|c|c|c|c|c|c|}
\hline & \multicolumn{4}{|c|}{ PSI-Maximum } & \multirow{2}{*}{ gesamt } \\
\hline & & & 0 & 1 & 2 & 3 & \\
\hline \multirow{6}{*}{ 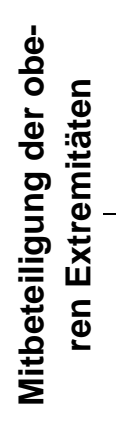 } & \multirow{3}{*}{$\stackrel{\frac{\varsigma}{\Phi}}{\subseteq}$} & Anzahl & 2 & 8 & 9 & 10 & 29 \\
\hline & & $\%$ innerh. Mitbet. oberer Gelenke & $7 \%$ & $28 \%$ & $31 \%$ & $34 \%$ & $100 \%$ \\
\hline & & $\%$ innerh. PSI-Maximum & $67 \%$ & $53 \%$ & $56 \%$ & $43 \%$ & $51 \%$ \\
\hline & \multirow{3}{*}{ 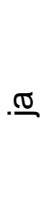 } & Anzahl & 1 & 7 & 7 & 13 & 28 \\
\hline & & $\%$ innerh. Mitbet. oberer Gelenke & $4 \%$ & $25 \%$ & $25 \%$ & $46 \%$ & $100 \%$ \\
\hline & & $\%$ innerh. PSI-Maximum & $33 \%$ & $47 \%$ & $44 \%$ & $57 \%$ & $49 \%$ \\
\hline \multirow{3}{*}{ gesam } & & Anzahl & 3 & 15 & 16 & 23 & 57 \\
\hline & & $\%$ innerh. Mitbet. oberer Gelenke & $5 \%$ & $26 \%$ & $28 \%$ & $41 \%$ & $100 \%$ \\
\hline & & \% innerh. PSI-Maximum & $100 \%$ & $100 \%$ & $100 \%$ & $100 \%$ & $100 \%$ \\
\hline
\end{tabular}

Bezogen auf die einzelnen Gelenkbereiche war in den einzelnen Gruppen mit Mitbeteiligung der Finger-, Hand-, Ellenbogen- oder Schultergelenke im Gegensatz zu den jeweiligen Gruppen ohne Mitbeteiligung eine stets größere Patientenzahl mit einem PSI-Max von 3 zu finden (Abb. 9). Auffällig ist, dass bei Mitbeteiligung des Schultergelenkes ausschließlich PSI-Max-Werte von 2 oder 3 gemessen wurden. Allerdings wurde zwischen dem PSI-Max kein statistisch signifikanter Zusammenhang zu einer Mitbeteiligung der Gelenkbereiche „Finger" ( $\mathrm{Cl}=0,114$; $\mathrm{p}=0,862)$, „Handgelenk“ $(\mathrm{Cl}=0,280 ; \mathrm{p}=0,266)$, „Ellenbogen“ $(\mathrm{Cl}=0,254 ; \mathrm{p}=0,400)$ oder "Schulter" $(\mathrm{Cl}=0,166 ; \mathrm{p}=0,811)$ mittels des exakten Test nach Fisher ausgewiesen (Abb. 9). 


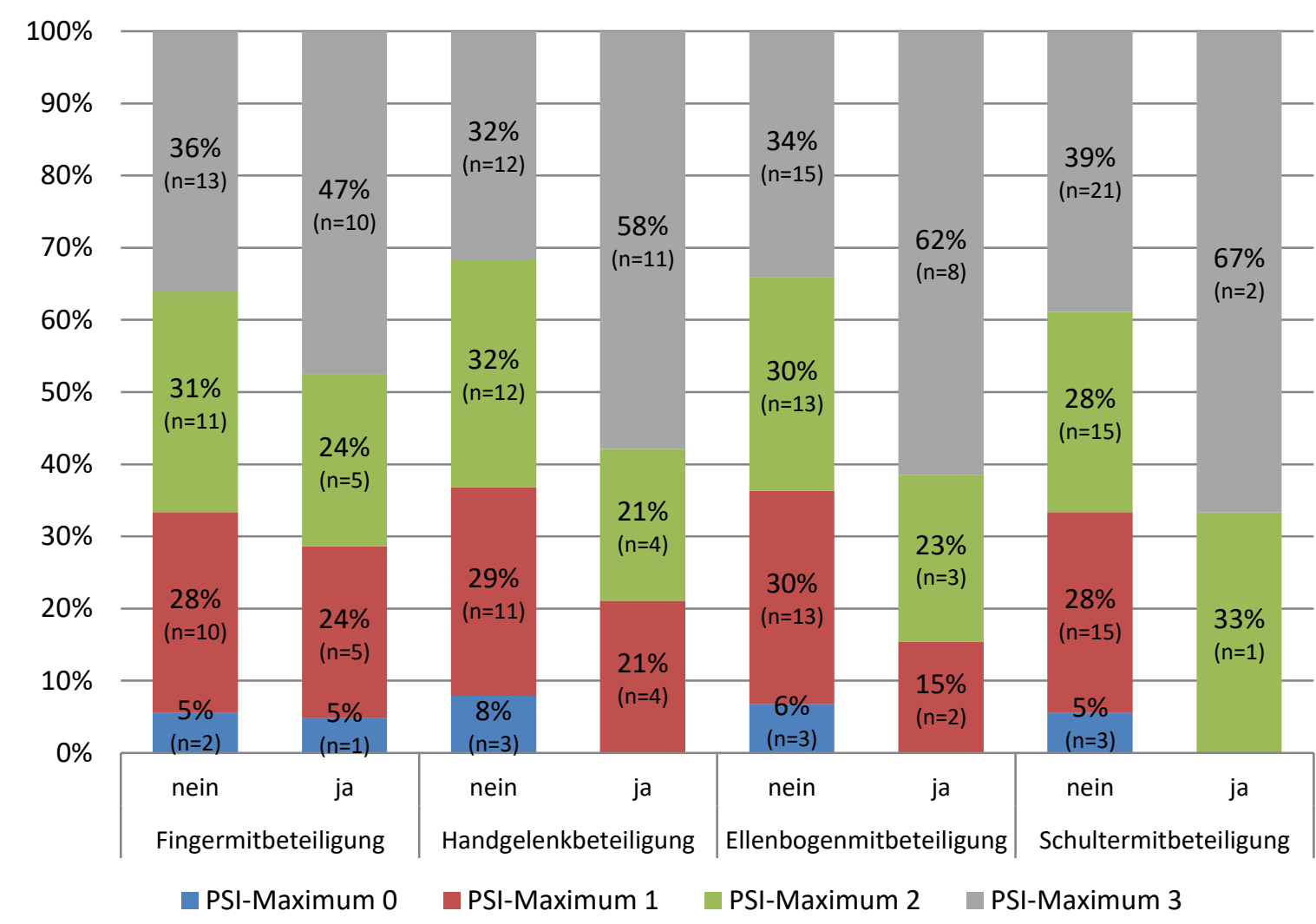

Abb. 9: Verteilung des maximal gemessenen PSI-Scores (PSI-Maximum) in $\%$ und absolut in Abhängigkeit von der Gelenkmitbeteiligung

\subsubsection{Vergleich von Parametern der oralen Gesundheit und röntgenologi- scher Auffälligkeiten in Abhängigkeit von Blutparametern}

Der Vergleich der mittleren PBI-Werte zeigte, dass Patienten mit erhöhtem CRP signifikant höhere PBI-Mittelwerte als Patienten mit normalem CRP aufwiesen. Für die immunologischen Marker ist auffällig, dass HLA-B27- und ANA-positive Patienten im Vergleich zu den Gruppen mit HLA-B27- und ANA-negativen Patienten niedrigere PBI-Werte zeigten. Für den Blutparamater ANA war dieser Unterschied signifikant ( $\mathrm{p}=0,011)$ (Tab. 24).

Bezogen auf den PSI-Maximum zeigte sich, dass CRP-positive Patienten tendenziell auch erhöhte PSI-Max-Werte aufwiesen. Dies traf für die immunologischen Marker (HLA-B27 und ANA) nicht zu. Insbesondere bei ANA-positiven Patienten war der Patientenanteil mit PSI-Max-Werten von 3 deutlich geringer als bei ANAnegativen Patienten. Gleiches gilt für HLA-B27 (Abb. 10). Laut verwendetem exaktem Test nach Fisher konnte kein signifikanter Zusammenhang zwischen PSI-Max und den Blutparametern "CRP“ $(\mathrm{Cl}=0,183 ; \mathrm{p}=0,676)$, „HLA-B27“ $(\mathrm{Cl}=0,182$; $p=0,879)$ und „ANA“ $(C l=0,369 ; p=0,077)$ festgestellt werden (Abb. 10). 
Tab. 24: Statistische Auswertung des Zusammenhangs zwischen Blutparametern und dem oralen Entzündungsindex PBI

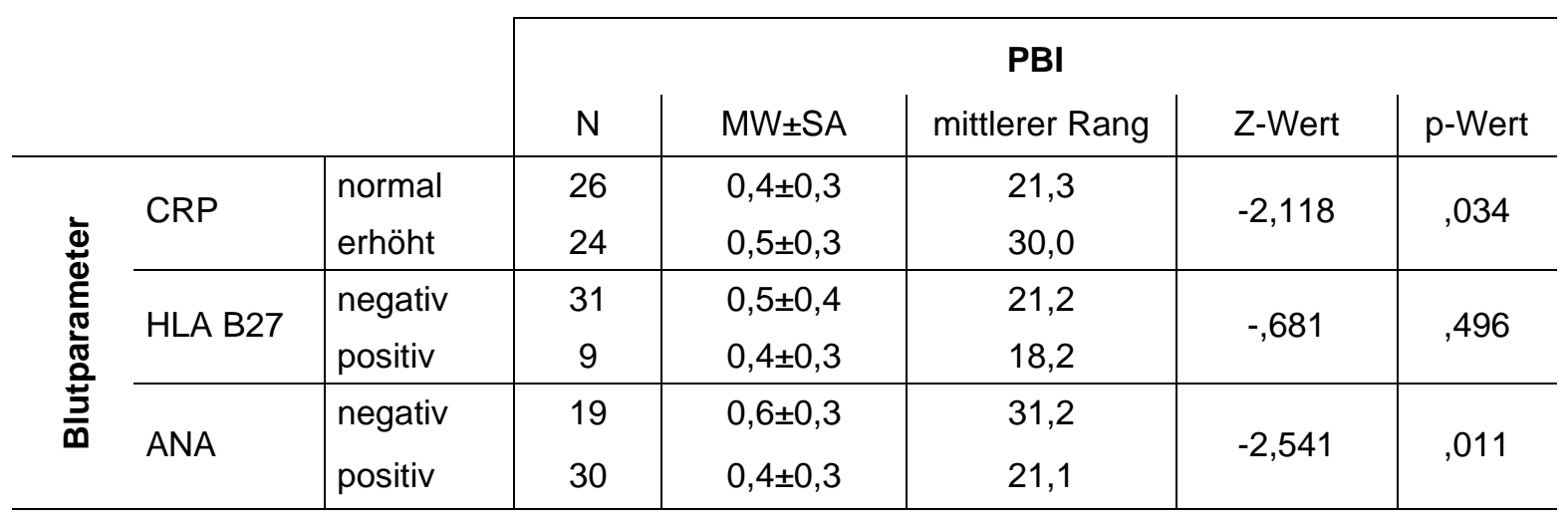

$\mathrm{MW}=$ Mittelwerte; $\mathrm{SA}=$ Standardabweichung; $\mathrm{CRP}=\mathrm{C}$-reaktives Protein; HLA = human leukoc cyte antigen, antinukleäre Antikörper; statistischer Test = Mann-Whitney-U

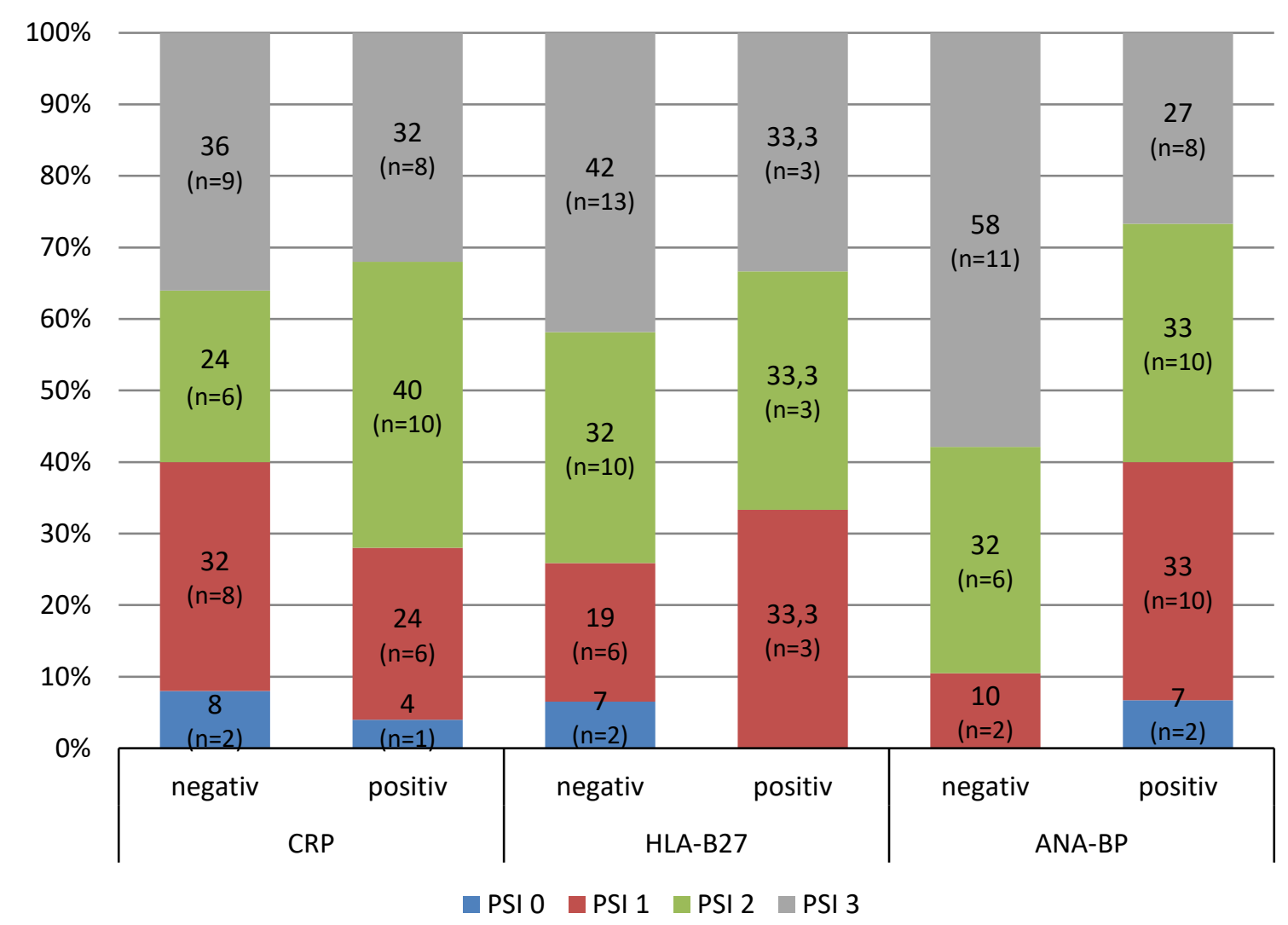

Abb. 10: Verteilung des maximal gemessenen PSI-Scores (PSI-Maximum) in \% und absolut in Abhängigkeit von den Blutparametern

Bei der Analyse der Blutparameter in Zusammenhang zum Röntgenscore (RS) zeigte sich, dass HLA-B27-positive Patienten vermehrt RS-Werte von 0 oder 1 als Patienten mit negativem HLA-B27-Wert aufwiesen (Abb. 11). Hingegen fanden sich bei den ANA-positiven Patienten bzw. Patienten mit erhöhten CRP-Werten tendenziell größere Anteile mit RS-Werten von 2 und 3 als bei ANA-negativen $\mathrm{Pa}$ - 
tienten bzw. Patienten mit unauffälligen CRP-Werten. Eine signifikante Abhängigkeit vom RS mittels des exakten Test nach Fisher konnte jedoch nur für das CRP $(\mathrm{Cl}=0,408 ; \mathrm{p}=0,043)$, nicht aber für HLA-B27 $(\mathrm{Cl}=0,234 ; \mathrm{p}=0,780)$ und ANA $(\mathrm{Cl}=0,157 ; \mathrm{p}=0,800)$ festgestellt werden.

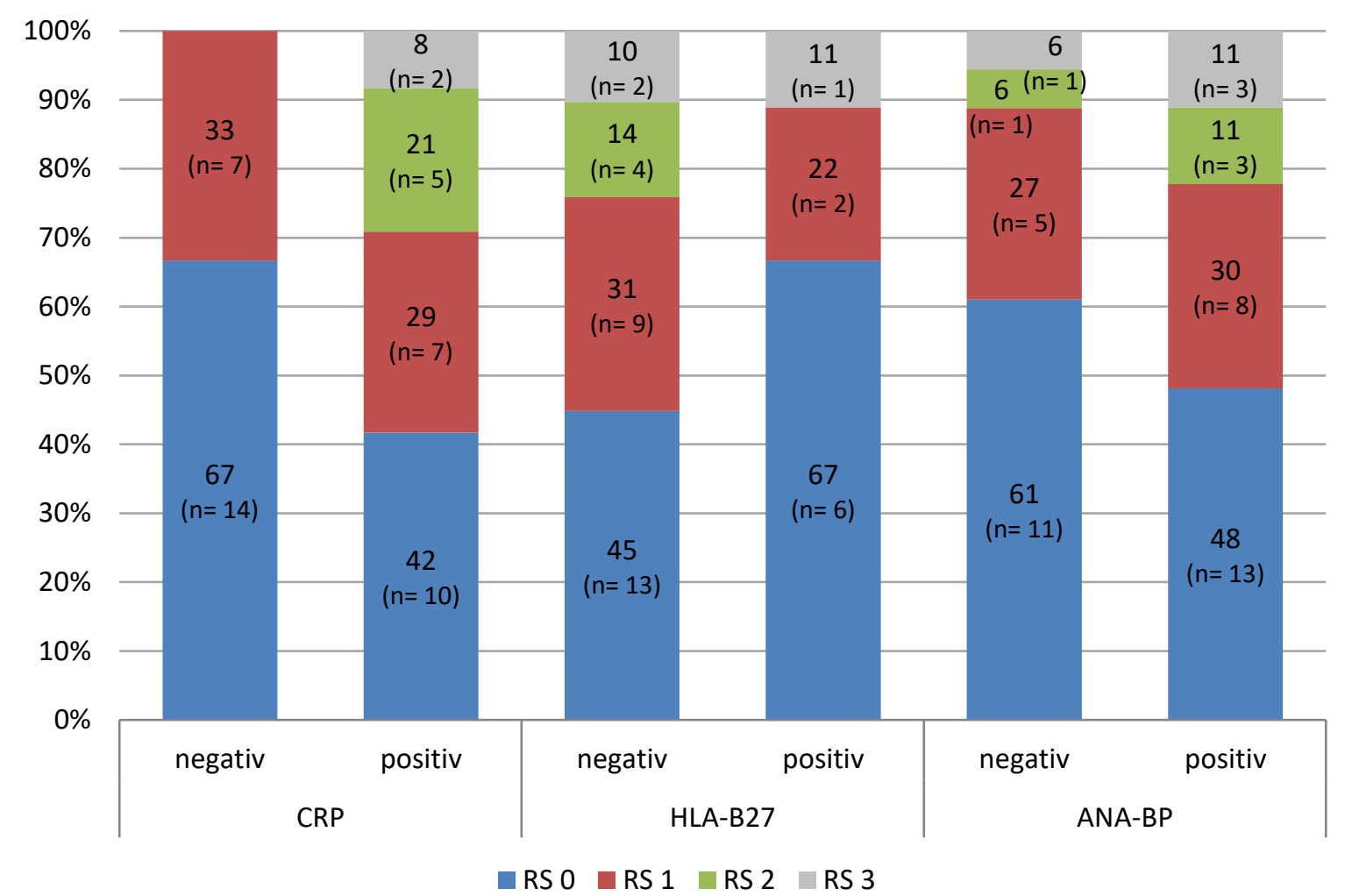

Abb. 11: Verteilung des Röntgenscores (RS) in \% und absoluten Zahlen in Abhängigkeit von den Blutparametern

\subsubsection{Parameter der oralen Gesundheit und röntgenologische Auffälligkei- ten in Abhängigkeit von der Erkrankungsdauer}

Abbildung 12 bis 14 stellen über Streudiagramme die Verteilung von dmf-t/DMF-Tsowie d-t/D-T und PBI-Maximum-Werte in Abhängigkeit von der Erkrankungsdauer dar. Bereits bei Patienten mit geringer Erkrankungsdauer zeigte sich eine große Streubreite von dmf-t/DMF-T, d-t/D-T und maximal gemessenen PBI-Werten. Ein linearer Zusammenhang konnte auch durch den Kendalls-Tau-Test nicht bestätigt werden. Hier wurden zwischen der Erkrankungsdauer und dem dmf-t/DMF-T ( $\mathrm{n}=$ 57, T= 0,054, $p=0,600)$, dem dt/DT ( $n=55, T=0,001, p=0,993)$ bzw. den PBIMaxima $(n=55, T=0,001, p=0,988)$ keine signifikanten Ergebnisse ausgewiesen. 


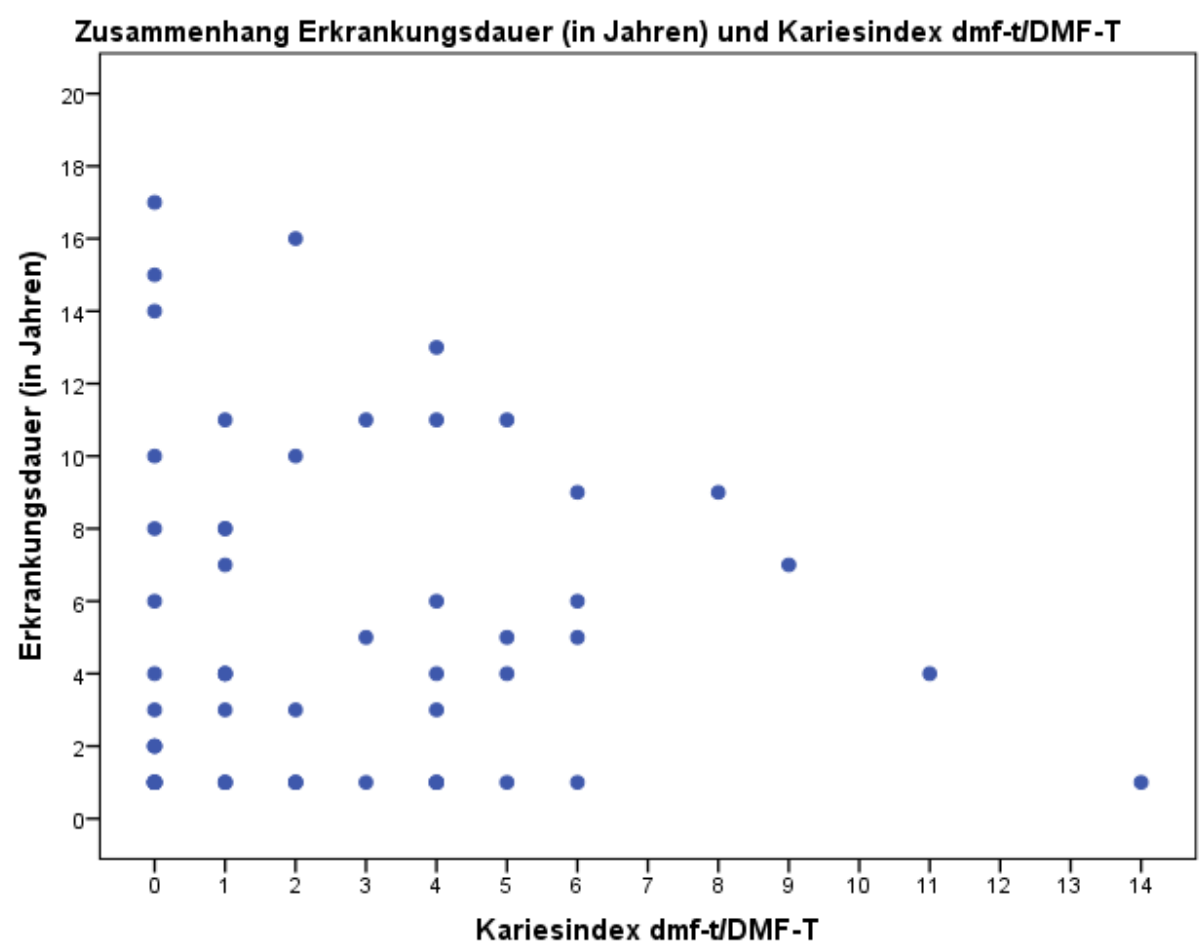

Abb. 12: Streudiagramm der Variablen Erkrankungsdauer (in Jahren) und Kariesindex dmf-t/DMF-T

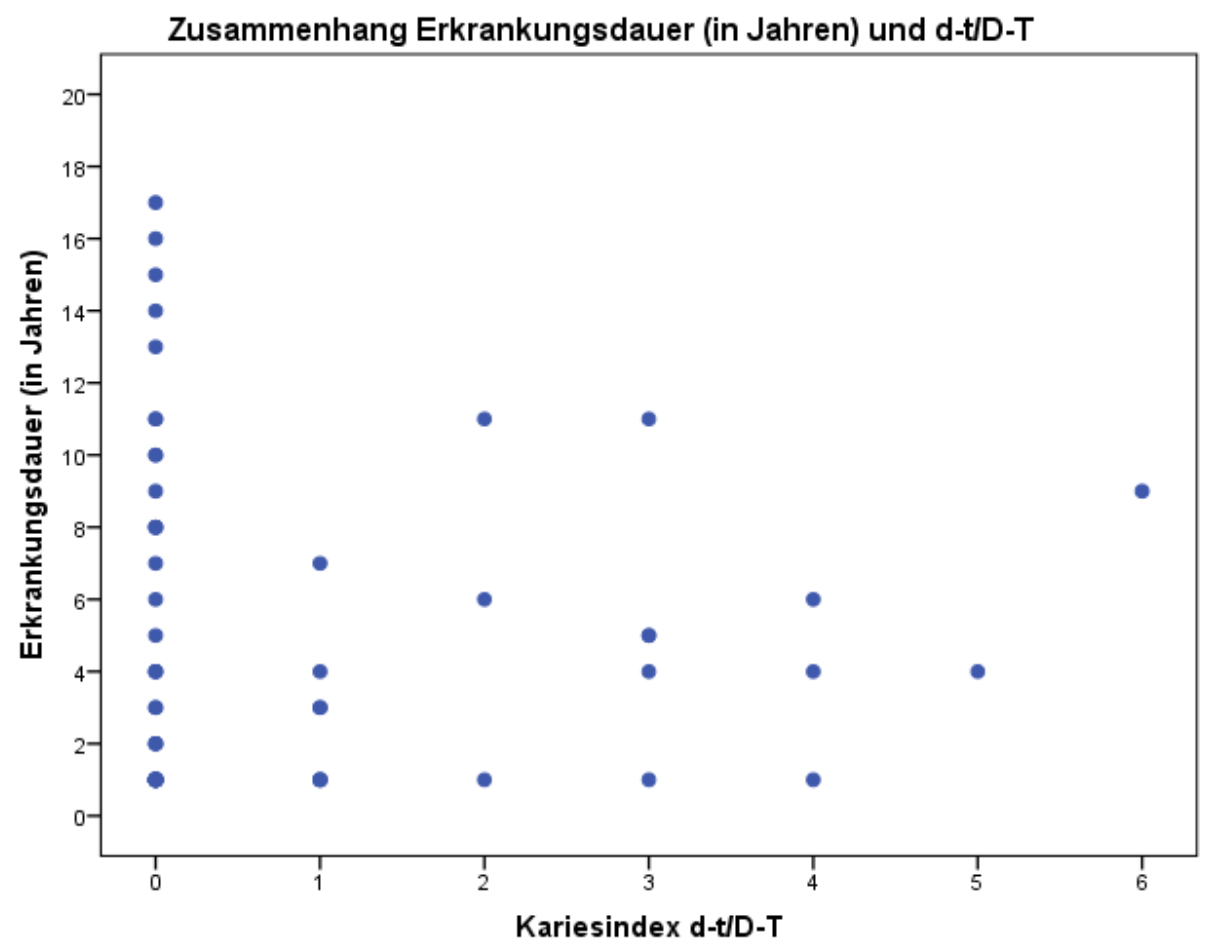

Abb. 13: Streudiagramm der Variablen Erkrankungsdauer (in Jahren) und Kariesindex d-t/D-T 


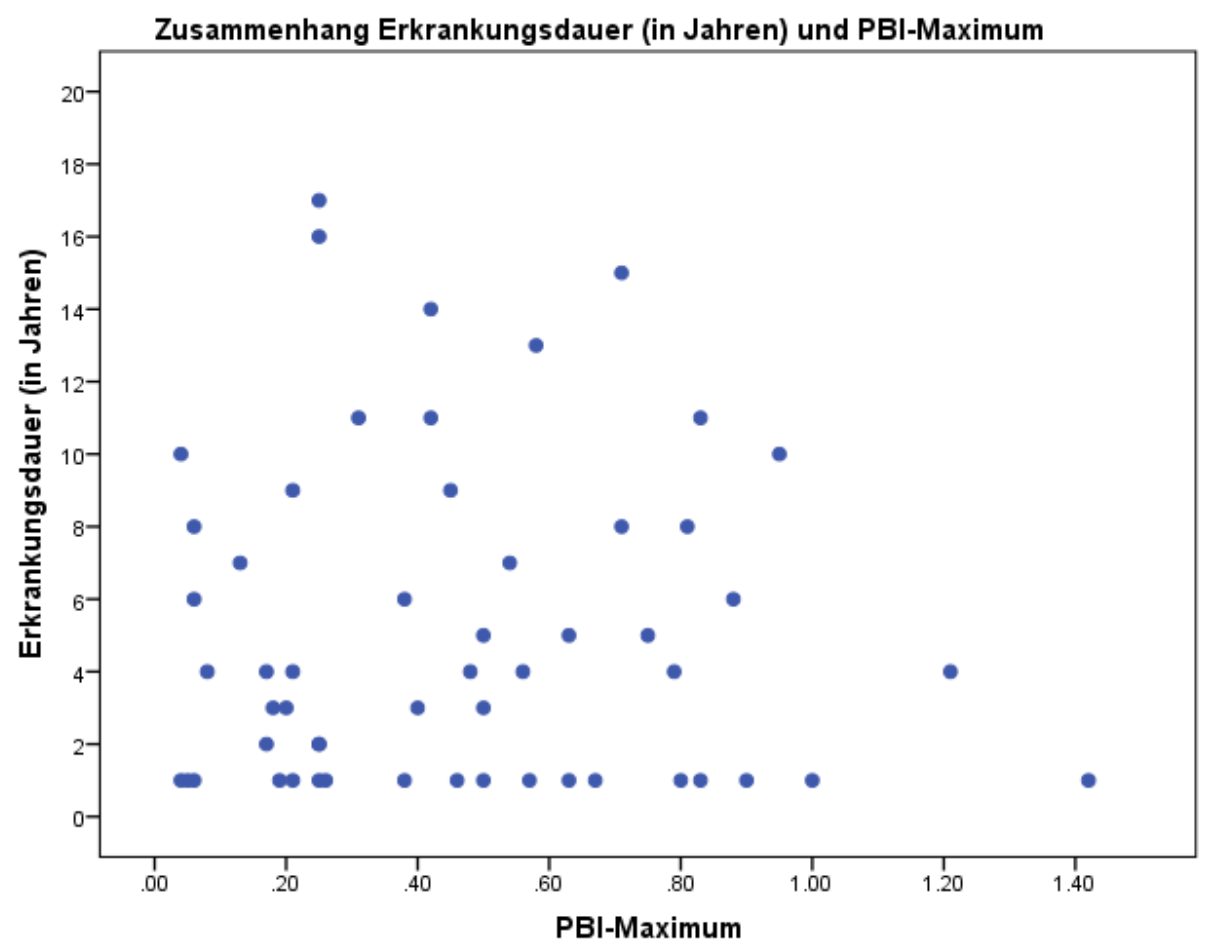

Abb. 14: Streudiagramm der Variablen Erkrankungsdauer (in Jahren) und PBI-Maximum

Bei Patienten mit einem maximalen PSI-Wert von 1 lag die höchste mittlere Erkrankungsdauer vor. Bezogen auf den Röntgenscore (RS) war ein deutlicher Anstieg der mittleren Erkrankungsdauer von RS 1 nach RS 3 zu beobachten. Die Mittelwertdifferenzen der Erkrankungsdauer waren allerdings insgesamt nicht ausgeprägt genug, um als statistisch signifikant ausgewiesen zu werden (Tab. 25).

Tab. 25: Mittelwertunterschiede der Erkrankungsdauer in Abhängigkeit vom PSI-Maximum und vom max. Röntgenscore

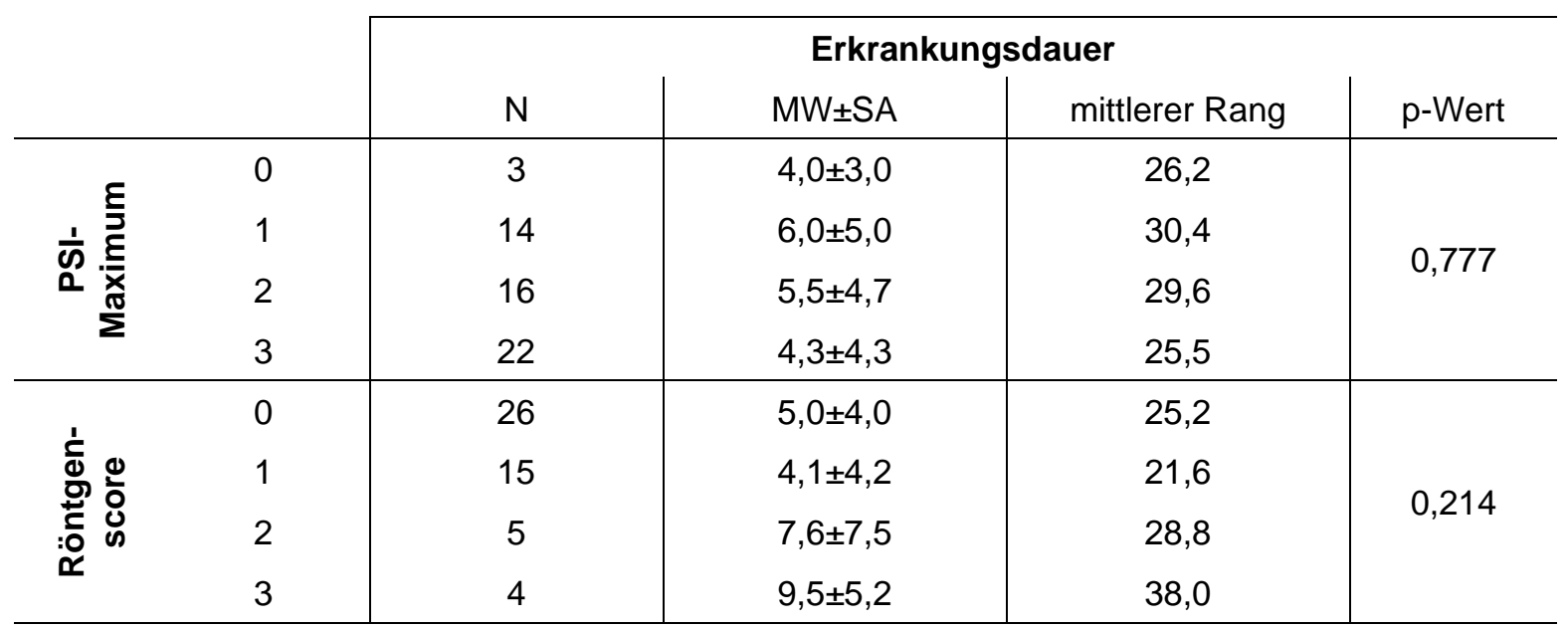

MW = Mittelwerte; SA = Standardabweichung; statistischer Test $=$ Kruskal-Wallis-Test 


\subsection{Untersuchungen zum Zusammenhang verschiedener Para- meter der Kiefergelenksdiagnostik}

\subsubsection{Zusammenhang zwischen anamnestischer und klinischer Kieferge- lenksdiagnostik}

Tabelle 26 zeigt in 75\% der Fälle eine Übereinstimmung von positiver Anamnese und positiver klinischer Diagnostik. Dieser positive Zusammenhang zwischen den beiden Variablen stellte sich statistisch signifikant dar $(\mathrm{Cl}=0,372, \mathrm{p}=0,007)$.

Tab. 26: Zusammenhang zwischen Anamnese und klinischer Diagnostik

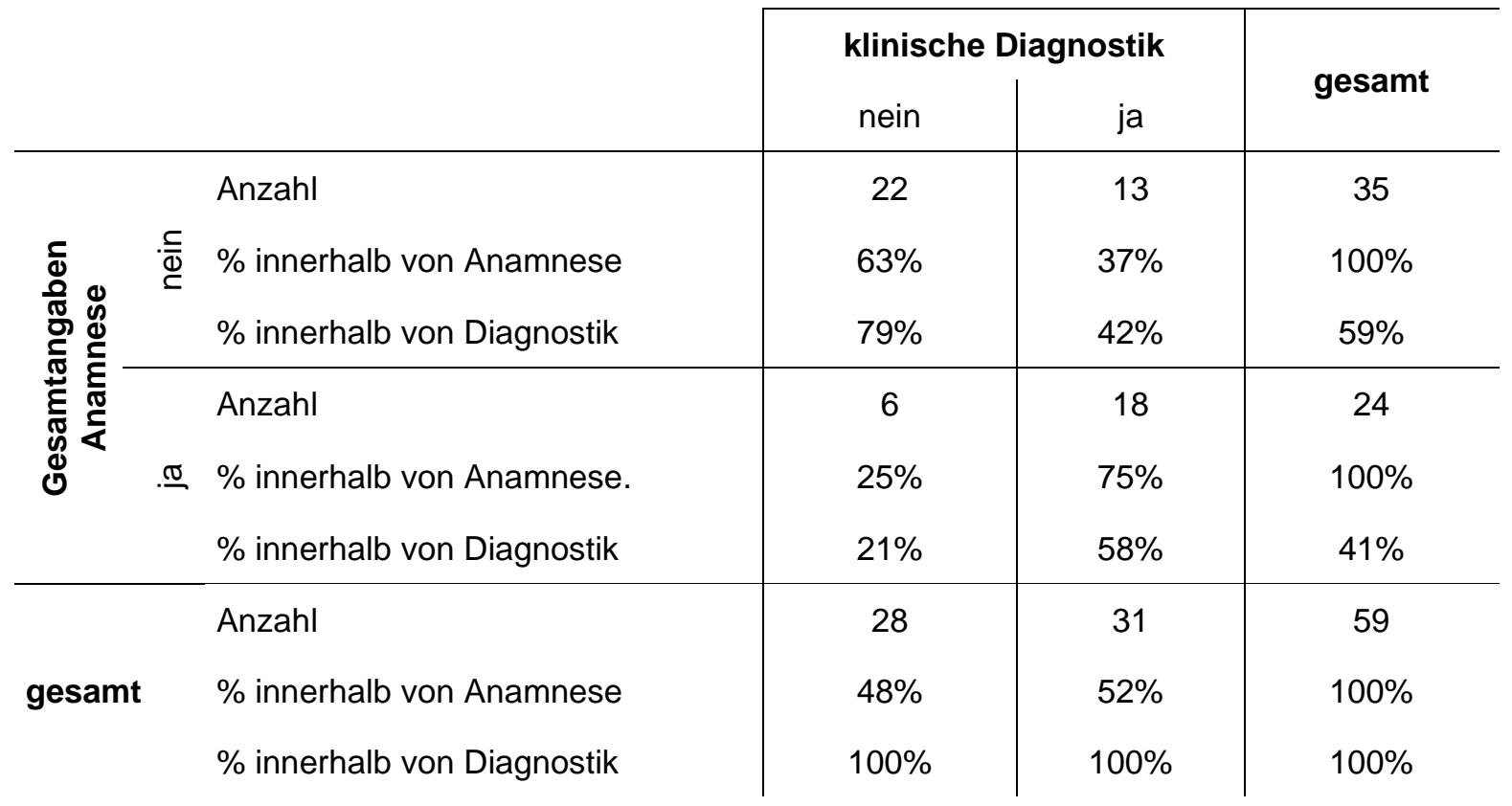

Bei den Zusammenhangsanalysen einzelner Anamnese- und klinischer Diagnostikparameter zeigte sich mittels des Exakten Tests nach Fisher insbesondere für die eingeschränkte Mundöffnung (MÖ) ein signifikanter Zusammenhang $(\mathrm{Cl}=0,532, \mathrm{p}=0,001)$. Ein positiver Zusammenhang konnte auch zwischen der Schmerzanamnese und der klinischen Schmerzdiagnostik beobachtet werden $(\mathrm{Cl}=0,329, \mathrm{p}=0,027)$. Bei genauerer Betrachtung der Ergebnisse der Schmerzdiagnostik war allerdings festzustellen, dass Personen mit positiver Schmerzanamnese vermehrt eine negative, klinische Diagnostik aufwiesen. Für das Kiefergelenkgeräusch wurde statistisch kein signifikanter Zusammenhang zwischen Anamnese und Diagnostik ermittelt $(\mathrm{Cl}=0,190, \mathrm{p}=0,198)$. Das Balkendiagramm in Abbildung 15 gibt einen Überblick über die prozentuale Verteilung der ermittelten Diagnostikparameter für jede Ausprägung der Anamneseparameter. 


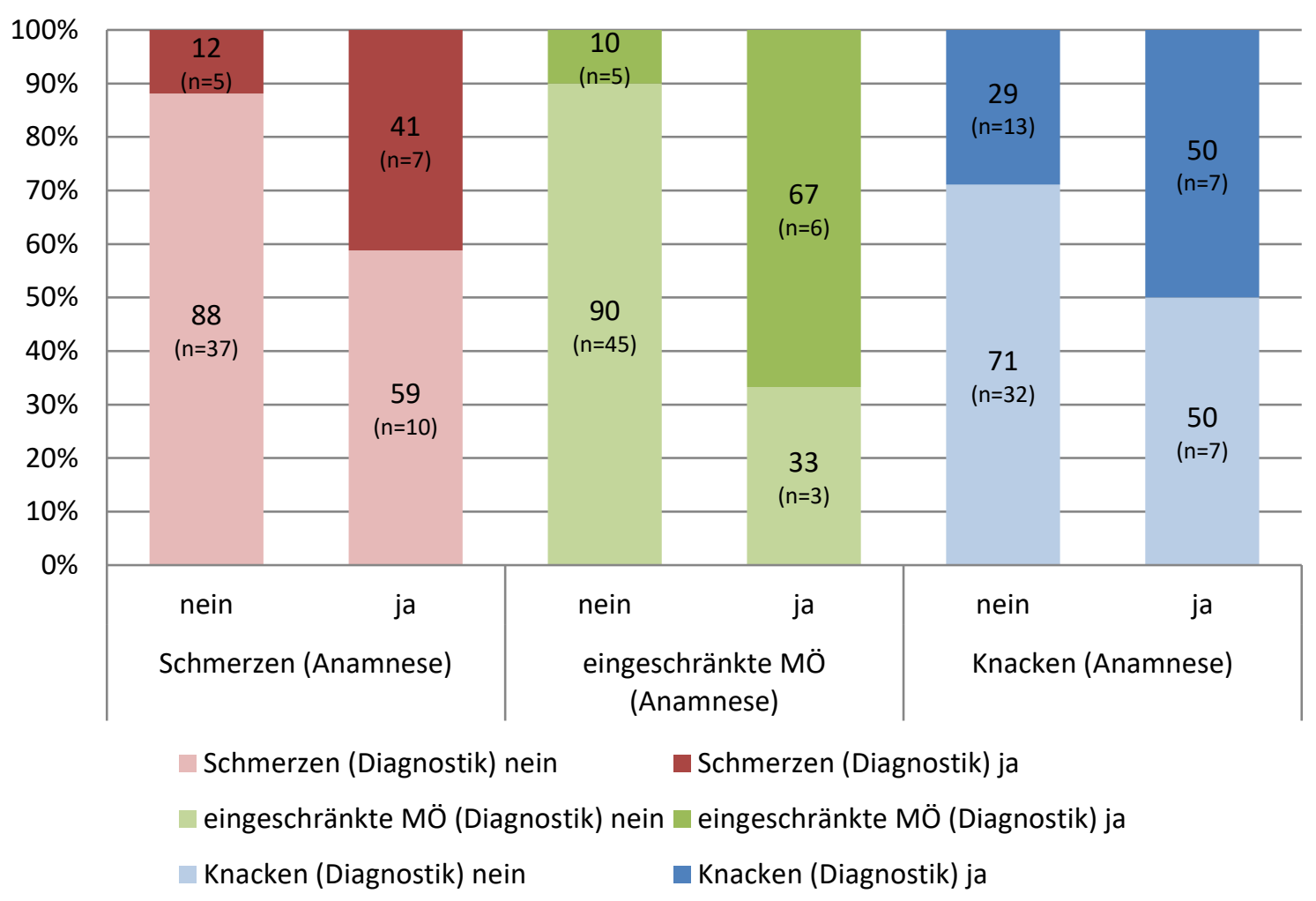

Abb. 15: Klinische Diagnostikparameter in Abhängigkeit von Anamneseparametern in \% und absolut

\subsubsection{Zusammenhang zwischen Röntgenscore und Anamnese}

58\% der Patienten mit einer röntgenologischen Auffälligkeit (RS 1-3) waren auch anamnestisch auffällig (Tab. 27). Ein signifikanter Zusammenhang zwischen erhöhten Röntgenscores und einer positiven Anamnese konnte mit Hilfe des exakten Test nach Fischer bestätigt werden $(\mathrm{Cl}=0,45, \mathrm{p}=0,01)$.

Tab. 27: Kreuztabelle zum Zusammenhang zwischen Anamnese und Röntgenscore

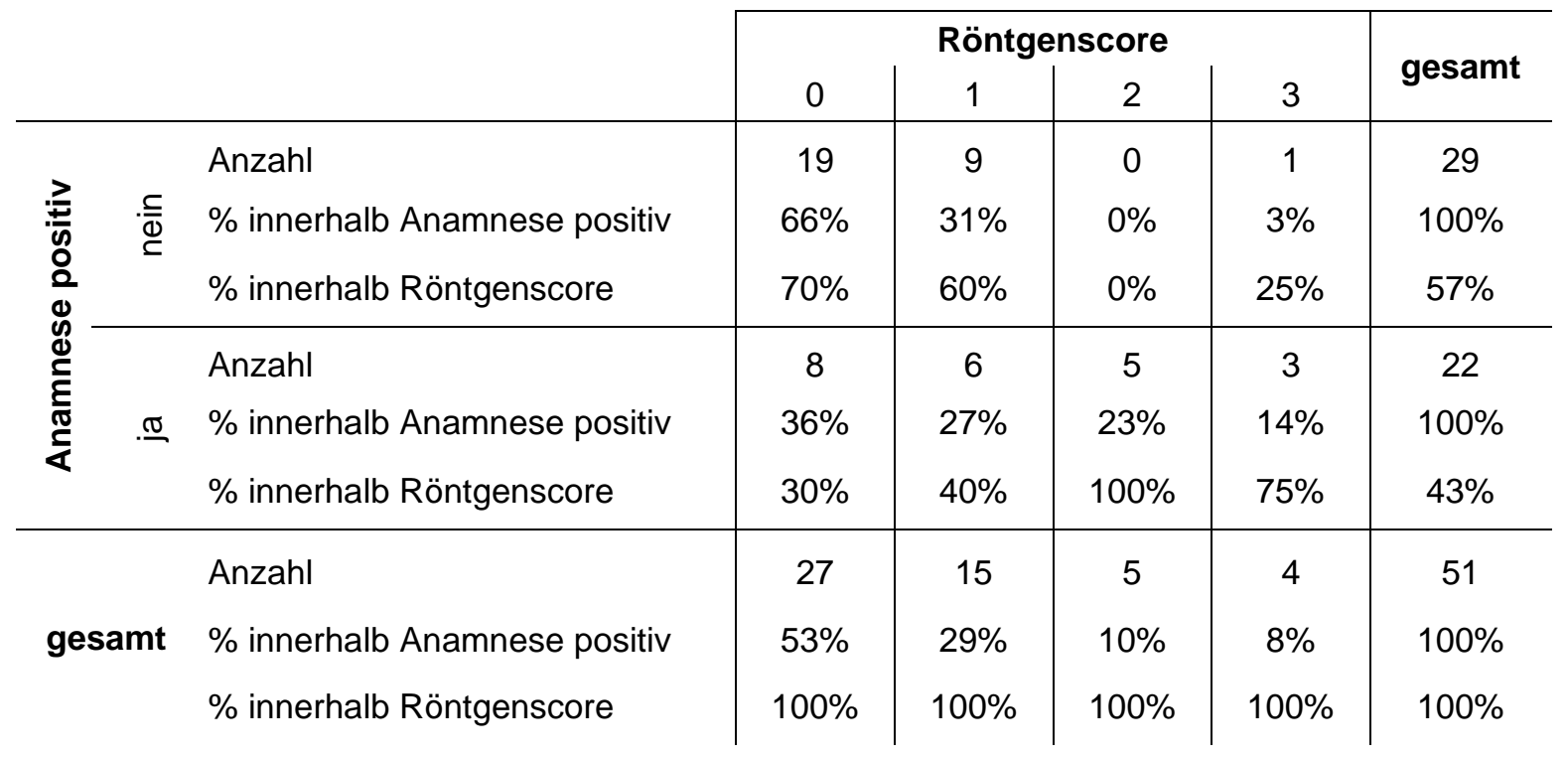


Die Abbildung 16 zeigt die Verteilung des Röntgenscores bei positiven und negativen Anamneseparametern. Lediglich für den Parameter „vorangegangene Blockade" konnte aufgrund der deutlich erkennbaren Häufigkeitsunterschiede zwischen niedrigen ( $\leq$ RS 1) und höheren Röntgenscores ( $\geq R S$ 2) ein signifikanter Zusammenhang ermittelt werden (exakter Test nach Fisher: $\mathrm{Cl}=0,598, \mathrm{p}=0,002$ ). Für die Parameter "Schmerzanamnese“ und „Kiefergelenkgeräusch“ war dies nicht der Fall (Schmerzanamnese_RS: $\quad \mathrm{Cl}=0,379, \quad \mathrm{p}=0,06$, Kiefergelenkgeräusch_RS: $\mathrm{Cl}=0,357, \mathrm{p}=0,07)$.

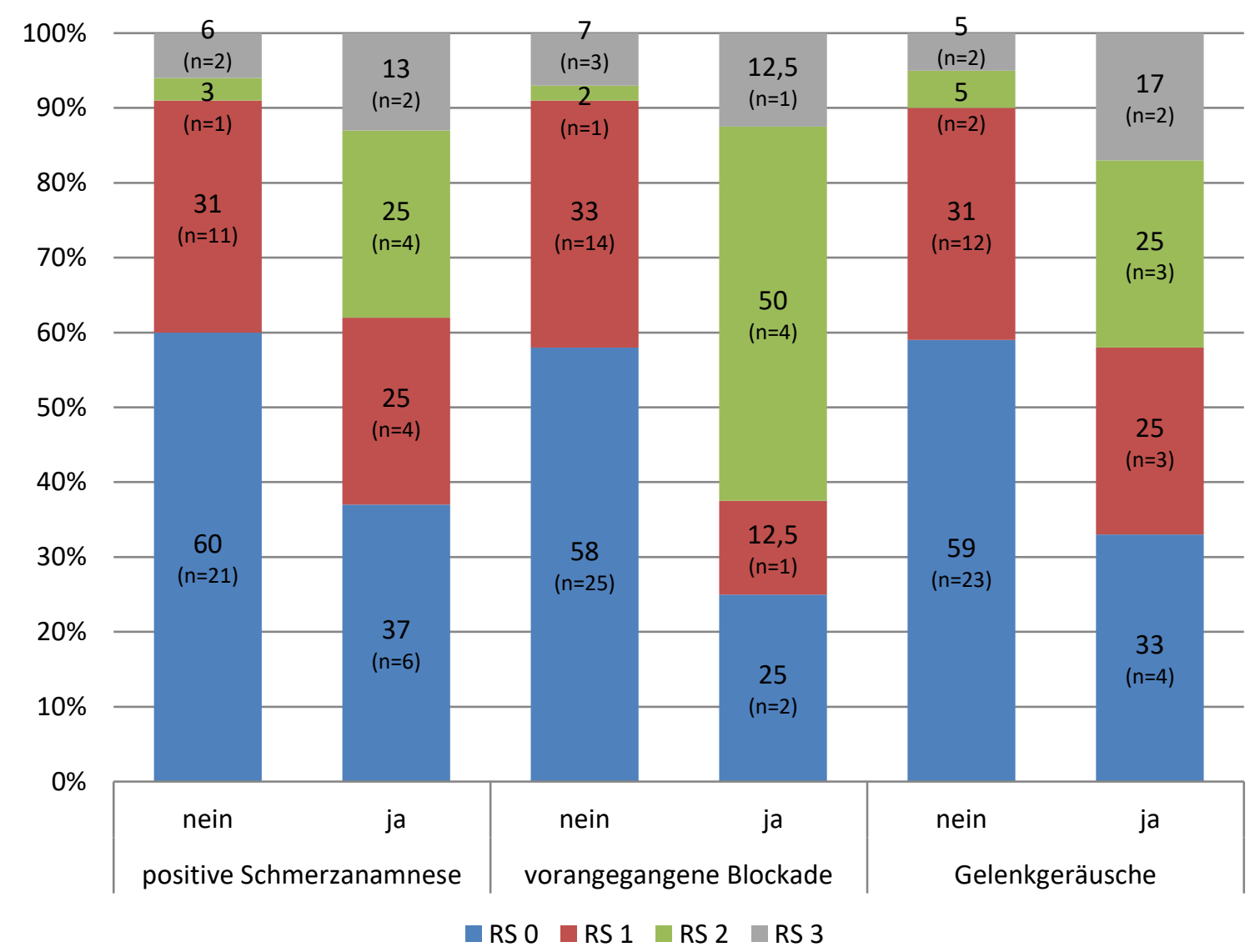

Abb. 16: Verteilung des Röntgenscores (RS) in Abhängigkeit von einzelnen Anamneseparametern

\subsubsection{Zusammenhang zwischen Röntgenscore und klinischer Diagnostik}

In dieser Analyse zeigten insgesamt $79 \%$ der Patienten mit einer röntgenologischen Auffälligkeit (RS 1-3) auch klinische Auffälligkeiten. Bei den Gruppen mit höheren RS-Werten waren signifikant mehr Patienten mit positiv ermittelter klinischer Diagnostik zu beobachten (exakter Test nach Fisher: $\mathrm{Cl}=0,479, \mathrm{p}=0,006$ ) (Tab. 28). Ausschließlich bei RS 0 überwog die Anzahl der Patienten mit unauffälliger klinischer Diagnostik (67\% zu 33\%). Dieses Verhältnis kehrte sich bereits bei 
RS 1 um und war für RS 2 und RS 3 mit einer Überzahl an Patienten mit positiver klinischer Diagnostik von $80 \%$ bzw. 100\% besonders ausgeprägt.

Tab. 28: Kreuztabelle zum Zusammenhang zwischen Diagnostik und Röntgenscore

\begin{tabular}{|c|c|c|c|c|c|c|c|}
\hline & \multicolumn{4}{|c|}{ Röntgenscore } & \multirow{2}{*}{ gesamt } \\
\hline & & & 0 & 1 & 2 & 3 & \\
\hline \multirow{6}{*}{ 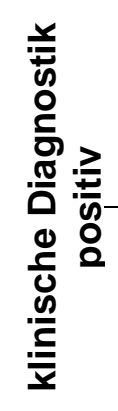 } & \multirow{3}{*}{$\cdot \frac{5}{0}$} & Anzahl & 18 & 4 & 1 & 0 & 23 \\
\hline & & \% innerhalb Diagnostik positiv & $78 \%$ & $18 \%$ & $4 \%$ & $0 \%$ & $100 \%$ \\
\hline & & \% innerhalb Röntgenscore & $67 \%$ & $27 \%$ & $20 \%$ & $0 \%$ & $45 \%$ \\
\hline & & Anzahl & 9 & 11 & 4 & 4 & 28 \\
\hline & . $\underline{\underline{\sigma}}$ & \% innerhalb Diagnostik positiv & $32 \%$ & $40 \%$ & $14 \%$ & $14 \%$ & $100 \%$ \\
\hline & & \% innerhalb Röntgenscore & $33 \%$ & $73 \%$ & $80 \%$ & $100 \%$ & $55 \%$ \\
\hline \multirow{3}{*}{\multicolumn{2}{|c|}{ gesamt }} & Anzahl & 27 & 15 & 5 & 4 & 51 \\
\hline & & \% innerhalb Diagnostik positiv & $53 \%$ & $29 \%$ & $10 \%$ & $8 \%$ & $100 \%$ \\
\hline & & \% innerhalb Röntgenscore & $100 \%$ & $100 \%$ & $100 \%$ & $100 \%$ & $100 \%$ \\
\hline
\end{tabular}

Betrachtet man die klinischen Diagnoseparameter im Einzelnen, stieg der Patientenanteil mit erhöhten RS-Werten für jeden positiv ermittelten Parameter tendenziell an (Abb. 17). Sowohl die eingeschränkte $M O ̈ ~(C l=0,592, p=0,001)$, wie auch die Gelenk- bzw. Muskelpalpation ( $\mathrm{Cl}=0,419, \mathrm{p}=0,015$ bzw. $\mathrm{Cl}=0,382, \mathrm{p}=0,034)$ und Kiefergelenkgeräusche $(\mathrm{Cl}=0,382, \mathrm{p}=0,034)$ zeigten laut exaktem Test nach Fischer signifikante Zusammenhänge mit zunehmenden RS-Werten. 


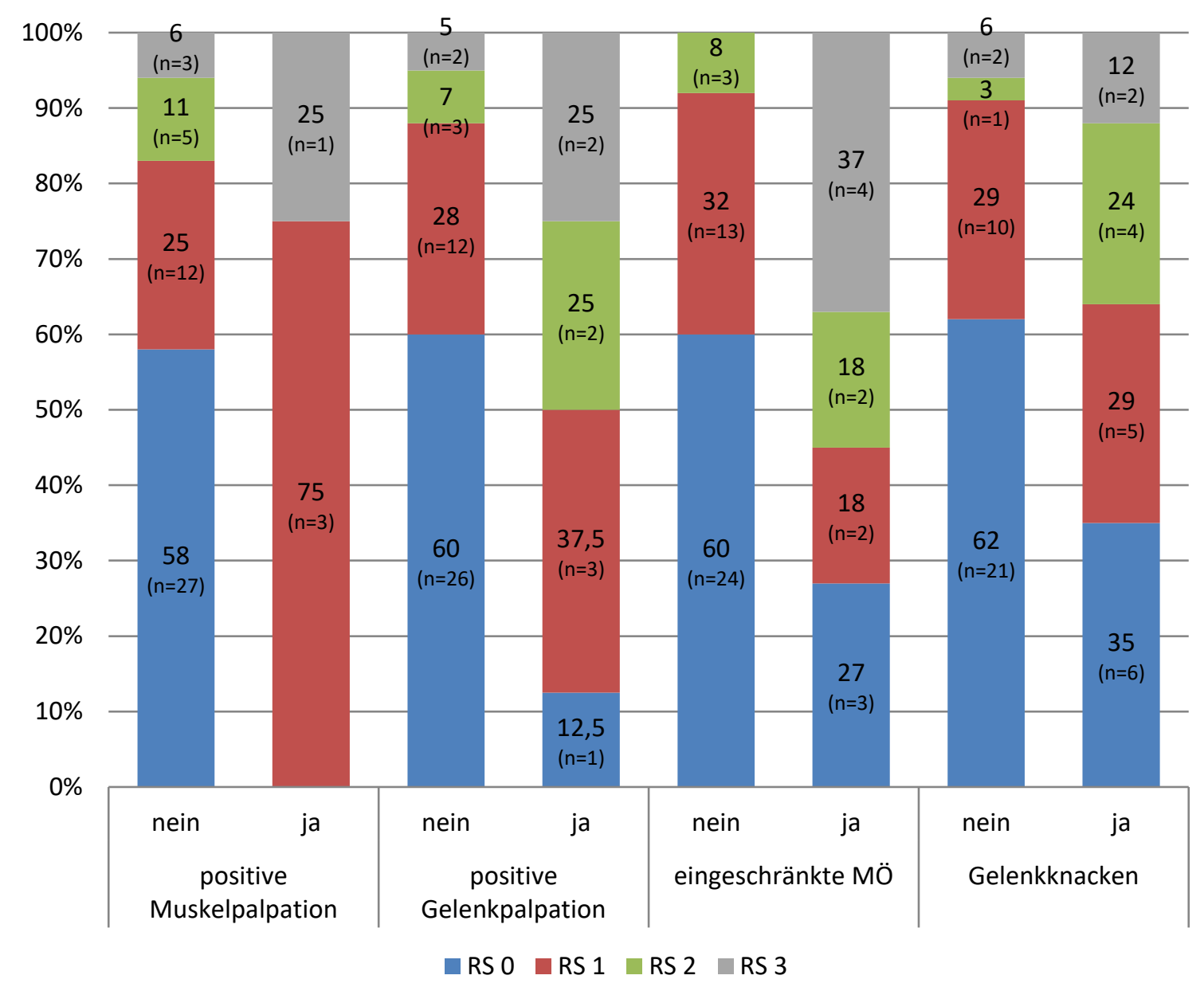

Abb. 17: Verteilung des Röntgenscores (RS) in Abhängigkeit von einzelnen klinischen Diagnostikparametern

\subsubsection{Zusammenhang zwischen Röntgenscore und einer Kombination aus Anamnese und klinischer Diagnostik}

Für die in Abbildung 18 dargestellten Untersuchungsergebnisse wurden diejenigen Patienten als insgesamt diagnostisch auffällig bezeichnet, wenn sie in mindestens einem der untersuchten Parameter der klinischen und/oder anamnestischen Diagnostik auffällig waren. Hierfür konnte ein signifikanter Zusammenhang zum RS ermittelt werden (Exakter Test nach Fisher: $\mathrm{Cl}=0,440, \mathrm{p}=0,022$ ). Patienten mit hohen Röntgenscores von 2 und 3 waren ausschließlich in der Patientengruppe mit diagnostischen Auffälligkeiten vertreten (Abb. 18). 


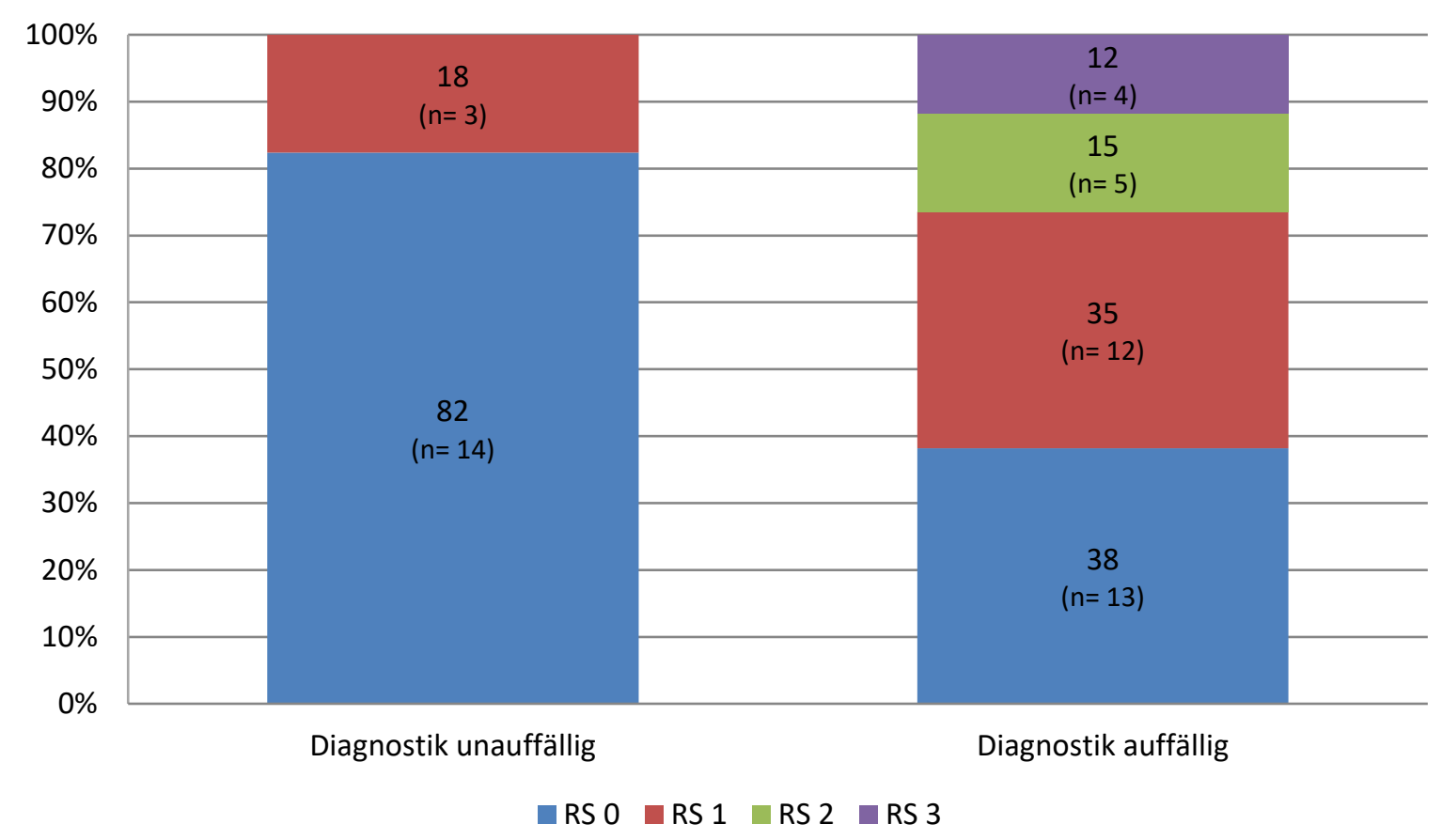

Abb. 18: Verteilung des Röntgenscores in Abhängigkeit von der Diagnostik (Kombination aus Anamnese und klinischer Diagnostik) in \% und absoluten Zahlen

Der Zusammenhang zwischen dem RS und den aus Anamnese und klinischer Diagnostik zusammengeführten Einzelergebnissen der untersuchten Parameter ist in Abbildung 19 ersichtlich. Für die Parameter "eingeschränkte Mundöffnung“ und "Schmerzen" bei der anamnestischen und/oder klinischen Diagnostik zeigten sich signifikante Zusammenhänge zum RS (exakter Test nach Fisher: $\mathrm{Cl}=0,717$, $\mathrm{p}=0,001$ bzw. $\mathrm{Cl}=0,426, \mathrm{p}=0,017)$. Für Gelenkgeräusche konnte kein signifikanter Zusammenhang zum RS errechnet werden $(\mathrm{Cl}=0,368, \mathrm{p}=0,073)$. 


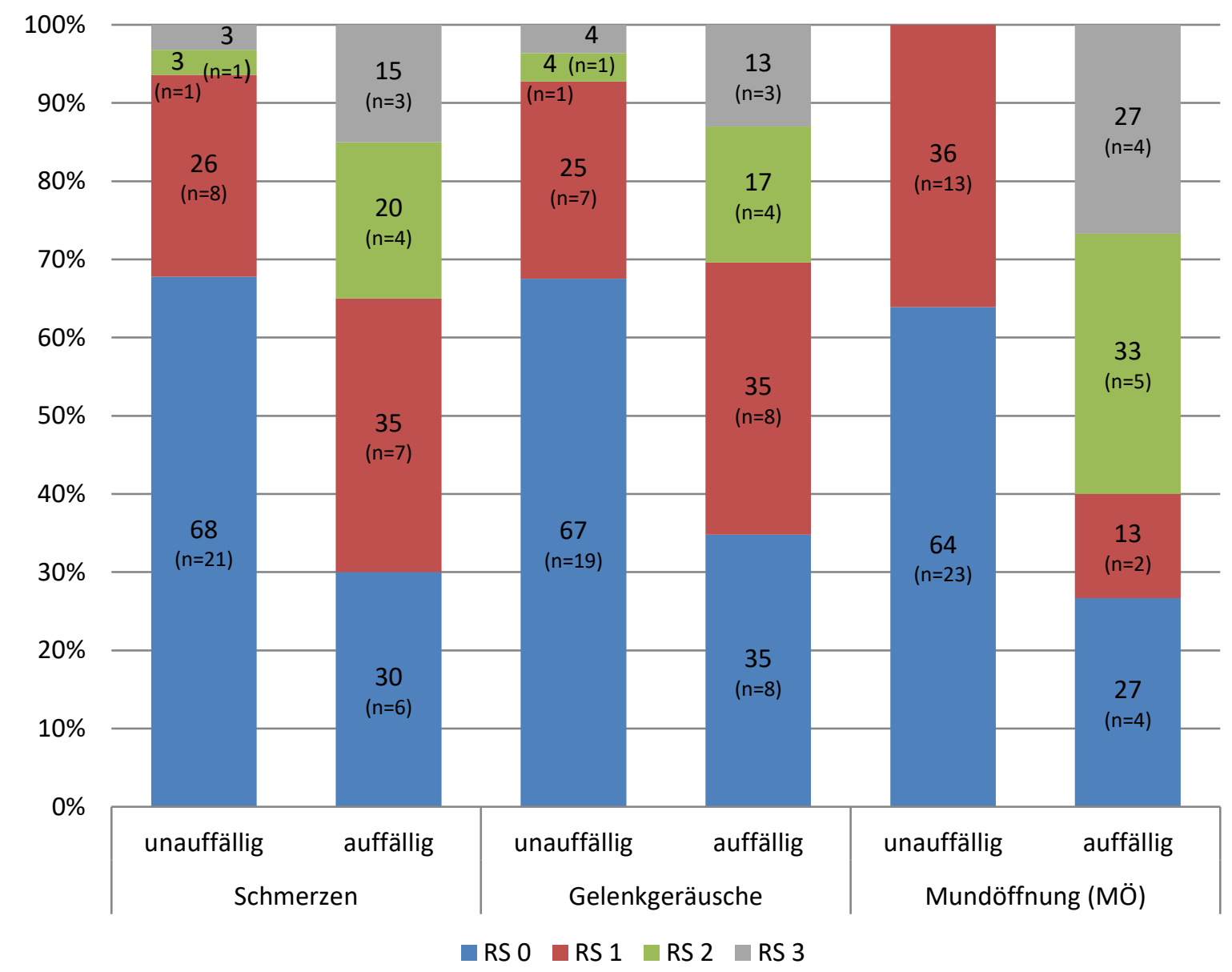

Abb. 19: Verteilung des Röntgenscores in Abhängigkeit von den einzelnen Diagnostikparametern (Kombination aus Anamnese und klinischer Diagnostik) in \% und absoluten Zahlen 


\subsection{Zusammenfassung der wichtigsten Ergebnisse}

Folgende Ergebnisse lassen sich zusammenfassen:

- Das Patientengut bestand aus 59 Patienten mit JIA (17 männlich und 42 weiblich).

- 37 Patienten wiesen eine Oligo- und 22 Patienten eine Polyarthritis auf.

- Der mittlere dmf-t/DMF-T betrug 2,6 $\pm 3,0$.

- Bei 26 Patienten wurden durch den PBI ausgeprägte Entzündungszeichen festgestellt (PBI-Max 3 und 4).

- Nach der PSI-Auswertung waren drei Patienten parodontal gesund, bei $39 \%$ waren Taschentiefen von 4 bis $5 \mathrm{~mm}$ zu sondieren.

- Bei der Befundung der OPG-Aufnahmen zeigten 24 Patienten Auffälligkeiten von dezent entrundeten Kondylen bis zu stark gekürzten oder fehlenden Kondylen.

- Bei der Kiefergelenksdiagnostik wiesen 24 Patienten anamnestische und 32 Patienten klinische Auffälligkeiten bzw. Befunde auf.

- Für die JIA-Parameter Erkrankungsform, Erkrankungsdauer, Beteiligung oberer Gelenke wurden keine signifikanten Einflüsse auf die Mund- und Kiefergelenksgesundheit festgestellt

- Ein signifikanter Zusammenhang zwischen Parametern der Kiefergelenksdiagnostik (Röntgenscore, schmerzhafte Kiefergelenke und eingeschränkte MÖ) und der Mundgesundheit konnte nicht ermittelt werden

- Bei ANA-negativen und Patienten mit erhöhten CRP-Werten waren deutlich verstärkte gingivale Blutungen zu beobachten. Es wurde ein Zusammenhang erhöhter CRP-Werte mit steigenden Röntgenscores (RS) beobachtet

- Lediglich für den Parameter der eingeschränkten MÖ waren signifikante Zusammenhänge zwischen der anamnestischen und der klinischen Kiefergelenksdiagnostik erkennbar

- Die positive Anamnese ging wie die positive klinische Diagnostik mit signifikant erhöhten Röntgenscores einher

- Für Patienten, die in mindestens einem der untersuchten Parameter der klinischen und/oder anamnestischen Diagnostik (Kombination aus Anamnese und klinischer Diagnostik) auffällig waren, wurden ausschließlich höhere Röntgenscores von 2 und 3 nachgewiesen. 


\section{Diskussion}

In dieser klinischen Querschnittsstudie wurden der Mundgesundheitszustand und der Zustand der Kiefergelenke bzw. Parameter der Kiefergelenksdiagnostik bei Kindern und Jugendlichen mit der Diagnose „JIA“ untersucht. Für auffällige Befunde der Mund- und Kiefergelenksgesundheit wurden zudem Zusammenhänge zu Patienten- und erkrankungsbezogenen Charakteristika ermittelt. Definierte Einund Ausschlusskriterien stellten die Grundlage für die Auswahl der Patienten dar (siehe Kap.4.2). Grundvoraussetzung war das Vorliegen einer diagnostizierten JIA, sowie die freiwillige Einwilligung der Patienten im Alter von 6-18 Jahren. Insgesamt konnten 59 Patienten für die Studie gewonnen werden.

Die OA ist mit 50-60\% der Patienten die größte JIA-Subgruppe (Kahn 2011) und war auch in der vorliegenden Arbeit mit $63 \%$ aller Patienten die überwiegende Erkrankungsart. Der deutlich größere Anteil von $71 \%$ weiblicher gegenüber $29 \%$ männlicher Patienten stellt hierbei eine zu erwartende Verteilung dar (Huang 2012, Kahn 2011). Die JIA ist als Erkrankung definiert, die vor dem 16. Lebensjahr beginnt (Hu et al. 2009). Das durchschnittliche Alter der hier untersuchten Patien-

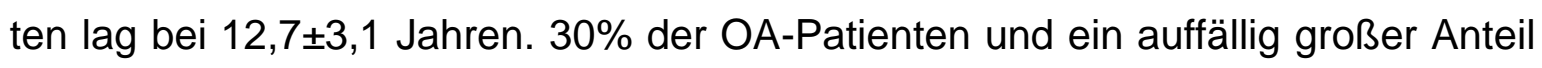
von $86 \%$ der Patienten mit einer PA wiesen auffällige Befunde in den Schulter-, Ellenbogen-, Hand-, oder Fingergelenken auf. Es konnten keine vergleichbaren Studienergebnisse gefunden werden, welche mitbeteiligte obere Gelenke bei einer JIA in ähnlicher Weise einteilten.

Der überwiegende Teil der Patienten von 83\% nahm nichtsteroidale Antirheumatika (NSARs) ein, überwiegend nach Bedarf, mit größeren oder kleineren Unterbrechungen und ohne genaue zeitliche Angabe. Zur schnellen Schmerzlinderung in Phasen des akuten Krankheitsgeschehens und oftmals bis zum Wirkungseintritt der DMARDs stellen die NSARs die Medikamentenklasse der ersten Wahl dar (Kumar und Banik 2013, Meier et al. 2013). Das Methotrexat ist das am häufigsten rezeptierte Medikament aus der Gruppe der DMARDs (Kumar und Banik 2013) und wurde auch bei der in der vorliegenden Studie untersuchten Patientengruppe nach den NSARs am häufigsten (46\%) eingenommen. Jedoch fehlten auch hier oftmals definierte Angaben zu Einnahmezeitpunkten. Andere Medikamente wurden von deutlich kleineren Anteilen der Patienten eingenommen. 
Bei den ermittelten Blutparametern war auffällig, dass nur bei einem Patienten ein positiver RF festgestellt wurde, während $44 \%$ der Patienten einen positiven CRP-Test und 53\% einen positiven ANA-Test aufwiesen. Das seltene Auftreten RF-negativer Patienten und die vergleichsweise hohen Anteile ANA- und CRP-positiver Patienten findet sich auch in einer weiteren Studienpopulation (Argyropoulou et al. 2009).

Hinsichtlich der oralen Gesundheit konnte in der vorliegenden Arbeit die in Kap. 2 postulierte Hypothese, dass JIA-Patienten einen dentalen und parodontalen Behandlungsbedarf aufweisen bestätigt werden. Bei den untersuchten JIA-Patienten

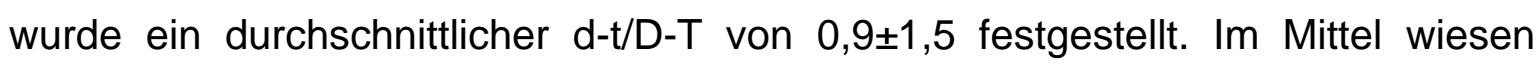
2,6 $\pm 3,0$ Zähne der Patienten eine Karieserfahrung (dmf-t/DMF-T) auf. Nach Micheelis und Schroeder (2006) können Kinder und Jugendliche mit einem DMF-T>2 als Risikogruppe angesehen werden. Verschiedene Arbeitsgruppen beschreiben signifikante Zusammenhänge zwischen der JIA und einem erhöhten Kariesvorkommen (Leksell et al. 2008, Savioli et al. 2004, Welbury et al. 2003). Andere Autoren konnten hingegen kein erhöhtes Kariesvorkommen bei JIA-Patienten feststellen (Feres de Melo et al. 2014, Ahmed et al. 2004). Ob der in dieser Studie ermittelte mittlere dmf-t/DMF-T-Wert gegenüber gesunden Kindern und Jugendlichen erhöht ist, kann nicht beantwortet werden, da keine Kontrollgruppe vorlag. Die fünfte deutsche Mundgesundheitsstudie offenbarte einen DMF-T bei 12Jährigen von 0,5 . 81,3\% der Kinder dieser Altersgruppe waren völlig kariesfrei (Jordan und Micheelis 2016). Im Vergleich lagen die Werte der hier untersuchten JIA-Patienten deutlich höher. Weitere Vergleiche der gewonnen Daten mit Beobachtungen anderer Untersuchungen scheinen nur schwer möglich. Zum einen liegt dies an den unterschiedlichen Studiendesigns. Bei einer der bisher größten Studie mit 149 Patienten wurde beispielsweise ausschließlich das Kariesvorkommen an bleibenden Zähnen von 6-17-Jährigen untersucht (Welbury et al. 2003). Hier lag der DMF-T bei JIA-Patienten bei 2,34 $\pm 2,86$ und in der Kontrollgruppe gesunder Patienten bei $2,16 \pm 2,8$. Zum anderen ist die Schwierigkeit im Vergleich verschiedener Studien in der großen Varianz ermittelter DMF-T-Werte zu sehen. Im Gegensatz zu Welbury et al. (2003) stellten Savioli et al. (2004) beispielsweise höhere mittlere DMF-T-Werte von 3,6 bei gesunden Kindern und Jugendlichen fest. Ein Grund hierfür könnte die generell unterschiedliche Karieserfahrung bei Patienten verschiedener Nationalitäten aufgrund verschiedenartiger Gesundheitssysteme sein. Weitere Fall-Kontroll-Studien zur Erfassung des dmf-t, des DMF-T 
oder des dmf-t/DMF-T bei Kindern und Jugendlichen mit JIA wären daher wünschenswert.

Im Weiteren wurde in einigen Studien ein vermehrtes Auftreten gingivaler Entzündungen bei JIA-Patienten beobachtet (Ahmed et al. 2004, Savioli et al. 2004, Welbury et al. 2003). Signifikante Zusammenhänge der JIA mit der Parodontitis wurden allerdings selten festgestellt (Hucke 2014). Das Fehlen einer Kontrollgruppe macht in der vorliegenden Studie eine Aussage bezüglich erhöhter Erkrankungswerte gegenüber gesunden Kindern und Jugendlichen nicht möglich. Jedoch wies lediglich ein Patient bei Durchführung des Papillen-Blutungs-Index (PBI) vollständig Blutungsfreiheit auf, während bei 43\% der Patienten sogar stärkere Blutungen (PBI 3 oder 4) beobachtet werden konnten. Die DMS V ermittelte bei einer gesunden, kindlichen Population ebenfalls maximale PBI-Grade und fand hierbei deutlich geringere Werte. Bei 12-Jährigen lag hier die Anzahl entzündungsfreier Gebisse bei 22\%, während ausgeprägte Entzündungsanzeichen (PBI-Grade 3 und 4) bei unter 10\% der Patienten vorkamen (Jordan und Micheelis 2016).

Die Auswertungen des PSI zeigten, dass lediglich drei Patienten in Bezug auf Parodontalerkrankungen „gesund“ (PSI-Wert 0) waren. Bei 39\% der Patienten waren Taschentiefen von 4 bis $5 \mathrm{~mm}$ (PSI-Wert 3) zu sondieren. In der DMS V wurde die Parodontitis bei Kindern nicht berücksichtigt, sodass auch hier ein Vergleich nicht möglich ist. Generell ist auf Basis der für diese Studie vorgenommenen Literaturrecherche festzustellen, dass anscheinend nur eine schwache und widersprüchliche Studienlage zur Detektion einer Parodontitis bei JIA-Patienten existiert. Während Miranda et al. (2003) von einem verstärktem Attachementverlust bei JIAPatienten gegenüber einer gesunden Kontrollgruppe berichten, konnten andere Autoren keine erhöhtes PD-Risiko von JIA-Patienten feststellen (Vahabi et al. 2015, Reichert et al. 2006). Bei der Beurteilung des PSI bei Kindern durch den Parameter „Taschentiefe“ ist grundsätzlich zu beachten, dass es sich bei erhöhten Werten auch um Pseudotaschen handeln könnte, die auf den Durchbruch von Zähnen im Wechselgebiss zurückzuführen sind (Heasman und Waterhouse 2012). Ungeachtet dessen sind tiefere Taschen durch die Mundhygienemaßnahmen des Patienten oftmals nicht mehr adäquat zu reinigen (Wolf et al. 2012). Entzündliche Vorgänge in solchen Taschen könnten durch die erhöhte Gefahr der Plaqueansammlung im Wechselgebiss nochmals begünstigt werden (Nobre et al. 2016, Wolf et al. 2012). Nach der Diagnosestellung einer JIA ist daher eine frühzeitige, 
zahnärztliche Untersuchung von besonderer Bedeutung, um entzündliche orale Erkrankungen, die womöglich einen Einfluss auf die Aktivität der JIA haben (Savioli et al. 2004), bei diesem Patientenklientel zu vermeiden.

Hinsichtlich der Kiefergelenksdiagnostik der hier untersuchten JIA-Patienten zeigten $41 \%$ anamnestische und $54 \%$ klinisch diagnostizierte Auffälligkeiten. Allerdings muss darauf hingewiesen werden, dass Anzeichen und Symptome von Kiefergelenkbeschwerden und Dysfunktionen auch bei gesunden Personen auftreten können. So konnten Koos et al. (2011) beispielsweise bei 30\% einer gesunden Patientengruppe Schmerzen im Mund-Kiefer-Gesichtsbereich feststellen und bei $18 \%$ eine eingeschränkte Mundöffnung (MÖ). Eine eingeschränkte MÖ wurde in dieser Studie gemäß verwendeter Definition bei 19\% der Patienten ( $n=11)$ festgestellt. Schmerzen in den Kiefergelenken oder der Kaumuskulatur traten in der klinischen Untersuchung bei 24\% der einbezogenen Patienten auf. Verschiedene Studien berichten in diesem Zusammenhang von klinisch diagnostizierten Gelenkund Muskelschmerzen bei einer Patientenanzahl zwischen $7 \%$ und $46 \%$ (Keller et al. 2015, Abramowicz et al. 2013, Pedersen et al. 2008, Weiss et al. 2008). Damit lag das in der vorliegenden Untersuchung ermittelte Ergebnis im Mittel vorliegender Studienerkenntnisse. Kiefergelenkgeräusche waren die klinisch am häufigsten zu beobachteten Auffälligkeiten bei 34\% der JIA-Patienten. Andere Autoren geben Werte von 6-41\% an (Keller et al. 2015, Koos et al. 2011, Weiss et al. 2008, Twilt et al. 2004). Somit liegen die hier festgestellten Werte ebenfalls in diesem Bereich. Bei der Anamnese wurden Kiefergelenkgeräusche und eine eingeschränkte MÖ seltener beobachtet als bei der klinischen Diagnostik (24\% und $15 \%$ ). Ein vermehrtes Auftreten klinischer gegenüber von Patienten selbst berichteten Auffälligkeiten konnten auch Cedströmer et al. (2013) belegen. Bezüglich der Schmerzanamnese waren in der vorliegenden Studie allerdings 29\% der Patienten auffällig, was den Anteil positiv klinisch diagnostizierter Schmerzen übertrifft. Andere Studien ermittelten oftmals geringere Patientenanteile, welche über Schmerzen in der Anamnese berichteten (Weiss et al. 2008, Arabshahi und Cron 2006, Twilt et al. 2004, Svensson et al. 2001).

Durch den oftmals asymptomatischen Verlauf der Kiefergelenkarthritis bei Kindern kommt der Analyse von OPG-Aufnahmen in der zahnärztlichen Praxis für die Diagnostik einer Mitbeteiligung der Kiefergelenke an der JIA eine große Bedeutung zu (Kahl-Nieke 2013). Bei der Auswertung der OPG-Aufnahmen wiesen nach den 
zu Grunde liegenden Röntgenscores 47\% der beurteilten JIA-Patienten morphologische Auffälligkeiten der Kiefergelenke auf. Ähnliche Werte für Kiefergelenkanomalien konnten auch anderen Studien ermitteln (Cedströmer et al. 2014, Cedströmer et al. 2013, Twilt et al. 2008, Twilt et al. 2004). Über die in diesen Studien berichteten Prozentangaben kondylärer Veränderungen besteht dabei eine große Spannbreite von 17-78\% (Karhulahti et al. 1993). Diese unterschiedlichen Ergebnisse kommen unter anderem durch die unterschiedlich verwendeten Diagnostik- und Scoresysteme zustande (Cedströmer et al. 2014, Karhulahti et al. 1993). Einige Autoren, wie z. B. Twilt et al. (2008), nutzten beispielsweise die sechs Kategorien nach Rohlin (Rohlin und Petersson 1989), während andere Autoren einen Score von 0-4 ( $\mathrm{Hu}$ et al. 2009) beziehungsweise 0-3 (Kahl-Nieke 2013) verwendeten. Die Entwicklung eines einheitlichen Scoresystems zur Bewertung von Röntgenbildern wäre folglich wünschenswert, um eine bessere Vergleichbarkeit der Studienergebnisse zu gewährleisten. In der vorliegenden Studie wurde in Anlehnung an die Untersuchungen von Kahl-Nieke (2013) ein Score von 0-3 verwendet.

Zum Weiteren waren in der vorliegenden Untersuchung Zusammenhänge zwischen Patienten- und Erkrankungscharakteristika, Parametern der oralen Gesundheit und der Kiefergelenksdiagnostik von Interesse. Hierdurch sollten patienten- und krankheitsbezogene Einflussfaktoren auf die Mundgesundheit und die Gesundheit der Kiefergelenke bei JIA-Patienten ermittelt werden. In Hinblick auf die Auswertungen zur oralen Gesundheit ließen sich keine signifikanten Unterschiede im Kariesvorkommen und der parodontalen Gesundheit zwischen OAund PA-Patienten erkennen. Einer der bedeutsamsten Gründe für orale Erkrankungen bei JIA-Patienten wird in einer eingeschränkten Mundhygiene infolge funktioneller Einschränkungen durch die Mitbeteiligung oberer Extremitäten gesehen (Savioli et al. 2004, Welbury et al. 2003, Walton et al. 1999, Tanchyk 1991). Savioli et al. (2004) zeigten einen signifikanten Zusammenhang zwischen der Anzahl an betroffenen oberen Gelenken und dem Vorkommen von Karies, Plaque und gingivalen Blutungen. $86 \%$ der PA-Patienten und 30\% der OA-Patienten hatten in der vorliegenden Untersuchung eine Mitbeteiligung mindestens eines Gelenkes der oberen Extremitäten. Allerdings ergaben die statistischen Analysen keinen signifikanten Zusammenhang einer Mitbeteiligung zu einem erhöhten Kariesrisiko oder parodontalen Auffälligkeiten. Lediglich in Bezug auf die Schultergelenke waren 
sowohl für den mittleren dmf-t/DMF-T, den mittleren $\mathrm{d}$-t/D-T als auch für die PSIMax-Werte Unterschiede zwischen den Patienten mit und ohne einer Beteiligung $\mathrm{zu}$ erkennen. Allerdings konnten auch diese Ergebnisse statistisch nicht gegen den Zufall abgesichert werden. Eine besondere Rolle des Schultergelenks lässt sich demnach durch die vorliegende Studie nicht sicher belegen. Die Beobachtungen dieser Studie könnten aber Anlass für weitere Untersuchungen geben, um den Einfluss unterschiedlicher Mitbeteiligungen oberer Gelenke auf die orale Mundhygiene und somit auf orale Erkrankungen bei dieser Patientenklientel zu untersuchen.

Für JIA-Patienten wird neben der Mitbeteiligung oberer Gelenke von einigen Autoren die eingeschränkte MÖ bei Mitbeteiligung der Kiefergelenke als weiterer funktionell bedingter Grund für ein gehäuftes Auftreten von Karies, bakteriellen Belägen und gingivalen Blutungen beschrieben (Savioli et al. 2004, Welbury et al. 2003). Bei den hier untersuchten JIA-Patienten mit einer eingeschränkten MÖ, röntgenologischen Kiefergelenkauffälligkeiten oder Schmerzen im Kiefergelenkbereich konnte allerdings kein vermehrtes Vorkommen dentaler oder parodontaler Erkrankungen festgestellt werden. Auch Ahmed et al. (2004) weisen auf Grundlage eigener Untersuchungen Bewegungseinschränkungen der Patienten nicht als primäre Ursache oraler Erkrankungen aus. Es könnte daher vermutet werden, dass sich möglicherweise die Eltern der JIA-Patienten über die Gefahr der eigeschränkten Mundhygiene in Folge von Bewegungseinschränkungen ihrer Kinder bewusst sind und die Mundhygiene verstärkt kontrollieren und unterstützen (Ahmed et al. 2004). Demnach wäre denkbar, dass andere Parameter, wie z. B. die Medikamenteneinnahme, eine veränderte Speichelzusammensetzung oder Blutparameter für ein vermehrtes Auftreten oraler Erkrankungen verantwortlich sind (Fabri et al. 2014, Reichert et al. 2007, Havemose-Poulsen et al. 2006, Walton et al. 2002, Siamopoulou et al. 1989).

In der vorliegenden Studie wurde auch ein eventueller Einfluss JIA typischer Blutparameter auf orale Erkrankungen überprüft. Diese weitere, nicht funktionell bedingte Assoziation betrifft allerdings weniger die Zahngesundheit (Karies), als vielmehr eine erhöhte Vulnerabilität des Zahnfleisches bzw. des Zahnhalteapparates (Reichert et al. 2007, Havemose-Poulsen et al. 2006). Sowohl bei HLA-B27positiven als auch ANA-positiven Patienten konnten in der vorliegenden Arbeit weniger deutliche orale Entzündungszeichen als bei HLA-B27- bzw. ANA- 
negativen Patienten beobachtet werden. Gingivale Blutungen wurden bei positiv getesteten antinukleären Antikörpern (ANA) sogar signifikant seltener festgestellt. Über ähnliche Zusammenhangsbeziehungen berichteten bereits andere Autoren, welche auf die Möglichkeit erhöhter Resistenzen gegenüber parodontopathogenen Vorgängen bei Exprimierung bestimmter HLA-Merkmale hinwiesen (Kaslick et al. 1975, Terasaki et al. 1975). Bei JIA-Patienten, welche erhöhte CRP-Werte aufwiesen, konnten in der vorliegenden Studie gegenüber Patienten mit normalen CRPWerten signifikant erhöhte gingivale Blutungen festgestellt werden. Dies bedeutet, dass im Untersuchungszeitraum sowohl verstärkte gingivale Entzündungszeichen als auch eine signifikant vermehrte Aktivität des Immunsystems zu beobachten waren. Diese Beobachtung könnte die Vermutung bestärken, dass bei der vorliegenden Patientengruppe Zusammenhangsbeziehungen zwischen Entzündungsgeschehen der JIA und parodontalen Erkrankungen in systemisch wechselwirkenden Krankheitsfaktoren zu suchen sein könnten.

In Bezug auf die Erkrankungsdauer wurden bei den Patienten keine signifikanten Unterschiede bei den Kariesbefunden, sowie den PBI- oder den PSI-Werten ermittelt. Mit Blick auf die Auswertungen zur Mitbeteiligung der Kiefergelenke bei JIAPatienten wäre zu erwarten gewesen, dass mit steigender Krankheitsdauer auch deutlich mehr Patienten unter Kiefergelenkerkrankungen leiden. Wenn auch nicht signifikant, so wiesen Patienten mit röntgenologischen Auffälligkeiten und zunehmenden Röntgenscores von 2 oder 3 eine Zunahme der mittleren Erkrankungsdauer auf. Allerdings war die mittlere Erkrankungsdauer bei Patienten mit RS 1 niedriger als bei röntgenologisch unauffälligen Patienten (RS 0). Signifikante Unterschiede bei den RS-Werten in Abhängigkeit von der Erkrankungsdauer konnten nicht festgestellt werden. Diese Ergebnisse könnten darauf hindeuten, dass eine fortgeschrittene Erkrankungsdauer nicht der ausschlaggebende Parameter für morphologische Veränderungen bzw. für Destruktionen der Kiefergelenke darstellt.

Patienten mit einer Polyarthritis tragen nach Cannizzaro et al. (2011) und Twilt et al. (2004) gegenüber Oligoarthritispatienten ein erhöhtes Risiko für die Erkrankung an einer Kiefergelenkarthritis. In der vorliegenden Untersuchung konnte festgestellt werden, dass fortgeschrittene Gelenkläsionen (RS 3) hauptsächlich bei PAPatienten zu beobachten waren ( $75 \%$ der Patienten mit einem RS von 3). Allerdings wurde Patienten mit einem Röntgenscore von 1 oder 2 häufiger eine $O A$ als 
eine PA zugeordnet. Somit kann die Annahme, dass vorwiegend Patienten mit einer PA röntgenologische Kiefergelenksauffälligkeiten aufweisen, durch diese Untersuchung nicht bestätigt werden. Eine frühzeitige Untersuchung der Kiefergelenke bei OA- wie PA-Patienten scheint demnach gleichermaßen notwendig. Auf Basis der Ergebnisse stellt sich jedoch die Frage, inwieweit Patienten mit dem Krankheitsbild der Polyarthritis gegenüber OA-Patienten ein erhöhtes Risiko insbesondere für fortgeschrittene Gelenkdestruktionen (RS 3) haben. Dies könnte Grundlage weiterer Studien sein.

Neben dem Einfluss der Erkrankungsart und der Erkrankungsdauer wird auch über Zusammenhänge zwischen dem Vorhandensein bestimmter Blutparameter und der Kiefergelenkarthritis berichtet. Verschiedene Studien beschreiben einen protektiven Einfluss von HLA-B27-Allelen auf die Kiefergelenkarthritis bei JIAPatienten (Dāvidsone et al. 2016, Cannizzaro et al. 2011). In der vorliegenden Untersuchung konnten keine Zusammenhänge zwischen HLA-B27 und Kiefergelenkauffälligkeiten aufgezeigt werden. Hierbei sei auf die im Vergleich zu Cannizzaro et al. (2011) $(n=226)$ deutlich kleinere Patientenanzahl der vorliegenden Studie hingewiesen. Durch die kleine Anzahl deutlich erkennbarer Kiefergelenkdestruktionen in Kombination mit der kleinen Anzahl HLA-B27 positiver Patienten waren kaum repräsentative, signifikante Ergebnisse zu ermitteln. Auffällig bei der hier untersuchten Patientengruppe ist allerdings der signifikante Zusammenhang des CRP mit positiven Kiefergelenksbefunden. So waren alle Patienten mit deutlichen morphologischen Kiefergelenksveränderungen und einem RS von 2 oder 3 ausschließlich CRP-positiv. Für CRP und ANA konnten Cannizzaro et al. (2011) keine Assoziationen zur Kiefergelenkarthritis feststellen. Eine Untersuchung an Erwachsenen konnte wiederum in Einklang mit den vorliegenden Ergebnissen einen Zusammenhang erhöhter CRP-Werte und der Kiefergelenkarthritis belegen (Celiker et al. 1995). Ein Zusammenhang zwischen dem Auftreten antinukleärer Antikörper (ANA) und der Kiefergelenkmitbeteiligung konnte in dieser Studie abschließend nicht festgestellt werden.

Zahlreiche Studien haben bereits klinische oder anamnestische Parameter der Kiefergelenksdiagnostik und deren Aussagekraft auf das Vorliegen einer Mitbeteiligung der Kiefergelenke bei JIA-Patienten untersucht (Keller et al. 2015, Abramowicz et al. 2013, Koos et al. 2011, Argyropoulou et al. 2009, Weiss et al. 2008, Billiau et al. 2007, Twilt et al. 2004). Es lagen bislang aber keine Informationen 
darüber vor, inwieweit klinisch diagnostizierte Parameter auch in der Anamnese von Patienten auffällig sind. In der vorliegenden Studie war festzustellen, dass sich JIA-Patienten in der klinischen Diagnostik teilweise vollkommen anders darstellten als bei der anamnestischen Befragung. In den Analysen zeigte sich für das vorliegende Patientenklientel, dass Personen mit positiver Schmerzanamnese gleichzeitig vermehrt eine negative Schmerzdiagnostik aufwiesen. Weiss et al. (2008) machten die Beobachtung, dass subjektive Symptome sehr spezifisch einen akuten Krankheitsverlauf anzeigen, während dies bei einem chronischen Geschehen nicht der Fall ist. Dies könnte erklären, warum in der vorliegenden Studie die Patienten, welche in der Anamnese über Schmerzen berichteten, zum Zeitpunkt der klinischen Untersuchung beschwerdefrei waren. Die Annahme, dass Patienten mit einer positiven Anamnese auch klinisch auffällig sind, konnte durch eine signifikante Übereinstimmung nur für den Parameter der eingeschränkten MÖ bestätigt werden. Dieses Ergebnis sowie die Ergebnisse zur Abhängigkeit der eingeschränkten MÖ vom Röntgenscore (s. o.), weisen die MÖ als einen der bedeutendsten diagnostischen Parameter bei der Kiefergelenksdiagnostik aus. Zu übereinstimmenden Bewertungen des Stellenwerts einer eingeschränkten MÖ kamen auch zahlreiche andere Untersuchungen (Keller et al. 2015, Saurenmann 2014, Müller et al. 2009, Twilt et al. 2008, Weiss et al. 2008, Stabrun et al. 1989).

Die aktuelle Literatur ergab sehr unterschiedliche Anhaltspunkte zu bedeutenden diagnostischen Parametern bei JIA-Patienten. In den Untersuchungen zum Zusammenhang diagnostischer Parameter und röntgenologischer Auffälligkeiten konnten Billiau et al. (2007) beispielsweise keinerlei Assoziationen feststellen. Andere Autoren fanden dagegen signifikante Zusammenhänge diagnostischer Parameter zu Kiefergelenkbeobachtungen in konventionellen Röntgenaufnahmen (Twilt et al. 2004, Stabrun et al. 1989). Die eingeschränkte MÖ wird oftmals als sicherster und einzig signifikanter Parameter genannt, eine Kiefergelenkarthritis zu diagnostizieren (Keller et al. 2015, Abramowicz et al. 2013, Stoll et al. 2012, Müller et al. 2009, Weiss et al. 2008). Im Gegensatz zu den oben aufgeführten Studien sollte in der vorliegenden Arbeit nicht nur ein möglicher Zusammenhang bestimmter Parameter zu einer Kiefergelenkerkrankung, sondern auch die Bedeutung anamnestischer gegenüber klinischer Diagnoseparameter der Kiefergelenkarthritis untersucht werden. Wie im Studienziel angenommen, bestand zu positiv diagnostizierten anamnestischen und/oder klinischen Parametern ein deutlicher Zusam- 
menhang zum Zerstörungsgrad der Kiefergelenke im OPG. Von den Patienten mit einer röntgenologischen Auffälligkeit (erhöhte RS-Werte) waren 79\% auch klinisch und 58\% anamnestisch auffällig. Ein vermehrtes Auftreten klinisch diagnostizierter gegenüber anamnestischer Auffälligkeiten bei vorliegender Kiefergelenkarthritis wird auch auf Grundlage der Untersuchungen von Koos et al. (2014) beschrieben. Bei der Untersuchung der interessierenden Parameter im Einzelnen war auffallend, dass für alle positiv untersuchten, klinischen Diagnoseparameter (Schmerzen, Kiefergelenkgeräusche, eingeschränkte MÖ) ein signifikanter Zusammenhang zu erhöhten RS-Werten bestand. Bei der Anamnese galt dies ausschließlich für den Parameter der eingeschränkten MÖ. Diese Erkenntnisse zeigen demnach die Notwendigkeit einer gut strukturierten Diagnostik, bestehend aus anamnestischen und klinischen Parametern. Zu beachten ist allerdings die eingeschränkte Aussagekraft der anamnestischen Befragung und der generell hohe Prozentsatz asymptomatischer Verläufe einer Kiefergelenkarthritis. Dies liegt unter anderem an der hohen Schmerzschwelle junger Patienten (Tzaribachev et al. 2010). So berichten JIA-Patienten von sich aus eher selten über Schmerzen im Mund-KieferGesichtsbereich, auch bei bereits fortgeschrittenen Kiefergelenkdestruktionen (Weiss et al. 2008, Arabshahi und Cron 2006, Svensson et al. 2001). Bei der klinischen Diagnostik fiel bei Patienten mit positiven Angaben zum Gelenkknacken der geringe Patientenanteil mit RS 3 auf. Dies könnte damit begründet werden, dass bei RS 3 oftmals eine eingeschränkte MÖ und auch das Fehlen einer Translationsbewegung vorliegen, sodass in diesem Stadium Knack- oder Reibegeräusche seltener entstehen.

Letztlich war bei Betrachtung jedes Parameters aus Anamnese und klinischer Diagnostik ein nicht unerheblicher Patientenanteil festzustellen, bei dem auch bei negativen Angaben fortgeschrittene Gelenkläsionen (RS 2-3) zu beobachten waren. Obwohl bei vielen Parametern ein signifikanter Zusammenhang zum Röntgenscore beobachtet werden konnte, legt dies nahe, dass es keinen Parameter unter den in dieser Studie verwendeten gibt, welcher für sich allein genommen eine Zerstörung der Kiefergelenke zuverlässig wiedergibt. Dies zeigt auch die Bedeutung bildgebender Verfahren. Ähnliche Beobachtungen wurden in OPG- und MRT-basierenden Studien gemacht, die über geringe Sensitivitätswerte einzelner Diagnostikparameter in Bezug auf das Erkennen einer Kiefergelenkarthritis berichten (Koos et al. 2014, Weiss et al. 2008, Twilt et al. 2004). Koos et al. (2014) be- 
schreiben allerdings eine deutliche Verbesserung der Sensitivitätswerte durch die Kombination mehrerer Parameter, wobei hier nur klinisch diagnostizierte Symptome berücksichtigt wurden. Daher verfolgte die vorliegende Studie im Weiteren das Ziel, den Zusammenhang einer Kombination gleichartiger anamnestischer und klinischer Diagnoseparameter in Bezug auf den RS zu untersuchen. Es sollte ermittelt werden, inwieweit Patienten, welche in OPG-Aufnahmen auffällig waren, auch in mindestens einem der Parameter Schmerzen, Kiefergelenksgeräusche und eingeschränkte MÖ eine Auffälligkeit zeigten - unabhängig davon, ob in Anamnese oder klinischer Diagnostik. Diesbezüglich waren $80 \%$ der Patienten mit einem Röntgenscore von 1 in der Anamnese und/oder der klinischen Diagnostik in mindestens einem der Parameter positiv und damit auffällig. Gleiches galt für alle Patienten mit einem RS von 2 oder 3. Auch hier muss insbesondere der Parameter "Mundöffnung" hervorgehoben werden. So waren alle Patienten, die keinerlei anamnestische und/oder klinische Auffälligkeiten bei der MÖ zeigten, auch röntgenologisch unauffällig oder wiesen lediglich geringgradige Kiefergelenkanomalien auf. Hohe RS-Werte von 2 oder 3 wurden bei diesen Patienten nicht gefunden. Wie bereits erwähnt und in Übereinstimmung zahlreicher Studien, weist auch diese Beobachtung auf eine starke Assoziation zwischen einer eingeschränkten MÖ und einer Kiefergelenksarthritis hin (Keller et al. 2015, Saurenmann 2014, Müller et al. 2009, Twilt et al. 2008, Weiss et al. 2008, Stabrun et al. 1989).

Die obigen Ergebnisse zeigen, dass JIA-Patienten mit einer auffälligen Röntgenauswertung auch fast ausschließlich eine positive Diagnostik in Anamnese und/oder klinischer Diagnostik aufwiesen. Allerdings muss beachtet werden, dass bei 38\% der Patienten mit einer positiven Diagnostik keinerlei röntgenologische Auffälligkeit beobachtet werden konnte. Eine positive klinische oder anamnestische Diagnostik geht demnach nicht zwangsläufig mit einer positiven Röntgendiagnostik einher. Eine gesichertere Aussage zur Stärke diagnostischer Parameter bei frühen, im OPG noch nicht sichtbarer Arthritisformen (RS 0) könnte allein durch die Zuhilfenahme der in der bildgebenden Kiefergelenksdiagnostik als Goldstandard angesehenen Magnetresonanztomographie getroffen werden (Keller et al. 2015, Carvalho et al. 2012, Müller et al. 2009, Weiss et al. 2008). Dessen ungeachtet erscheinen Untersuchungen wie die hier Vorliegende dennoch als bedeutsam. Die in der normalen Zahnarztpraxis verfügbaren Mittel zur Diagnostik einer Kiefergelenkerkrankung und deren Schweregrad sind die Anamnese, klini- 
sche Diagnostik sowie in den meisten Fällen das OPG. Nicht nur das im optimalen Falle frühzeitige Erkennen der Kiefergelenkmitbeteiligung ist wichtig, sondern auch das Erkennen bereits etablierter Läsionen ist von großer Bedeutung. So kann ein Fortschreiten der Destruktion durch geeignete Therapiemaßnahmen verhindert werden. Sogar eine Rehabilitation mit Ausheilung der Läsion scheint möglich (Twilt et al. 2008). Kahl-Nieke (2013) bestätigte das OPG als geeignetes Screening-Verfahren. Allerdings wurde hier nur auf die notwendige Sensibilisierung von Zahnärzten bezüglich der Analyse bereits vorhandener OPG-Aufnahmen hingewiesen. Aufgrund der Ergebnisse der vorliegenden Studie kann zur Diskussion gestellt werden, ob jeder JIA-Patient bei der zahnärztlichen Vorstellung geröntgt werden sollte oder ob die Indikationsstellung für ein Röntgenbild davon abhängig gemacht wird, inwieweit bereits in der Anamnese oder der klinischen Diagnostik eine Auffälligkeit zu erkennen war. Ob und in welcher Form das OPG in der Gesamtdiagnostik bei JIA-Patienten eingesetzt werden sollte, bleibt unklar und kann Grundlage weiterer Forschungsbestrebungen sein. 


\subsection{Stärken und Limitationen}

Bei der Diskussion und Einordnung der hier präsentierten Ergebnisse in den Forschungskontext muss schlussendlich auch auf bestehende Limitation und Stärken dieser Arbeit hingewiesen werden. Durch die Vielzahl der überprüften Parameter konnten insgesamt vielfältige Ansatzpunkte identifiziert werden, um den (zahn-) ärztlichen Umgang mit JIA-Patienten zu effektivieren. In Hinblick auf die Untersuchungen zwischen der oralen Gesundheit und der Mitbeteiligung oberer Extremitäten bei JIA-Patienten konnten keine vergleichbaren Studien gefunden werden, welche mitbeteiligte obere Gelenke in ähnlicher Weise einteilten. Dies kann als eine grundlegende Stärke der vorliegenden Untersuchungen angesehen werden. Selbiges gilt für die Kiefergelenksdiagnostik von JIA-Patienten. Auch hier konnte die Fragestellung über den Stellenwert anamnestischer gegenüber klinischer Diagnoseparameter aufgrund der mangelnden Studienlage bisher nicht beantwortet werden. Es lagen keine Informationen darüber vor, inwieweit klinisch diagnostizierte Parameter auch in der Anamnese von Patienten auffällig sind. Die vorliegende Arbeit versucht daher, einen Beitrag zur Verbesserung dieser Forschungslücken zu leisten. Damit ist als eine weitere Stärke dieser Untersuchung auch die sehr detaillierte Analyse von Zusammenhängen zwischen oralen Erkrankungen anamnestischen und/oder klinischen Diagnostikmöglichkeiten und den verschiedenen Zerstörungsgraden (RS-Werten) an Arthritis erkrankter Kiefergelenke hervorzuheben.

In Hinblick auf das methodische Vorgehen wurde bei der Befundung von Kiefergelenkanomalien und deren Zerstörungsgrade mittels OPG versucht, Fehler bei der Beurteilung der Röntgenbilder grundsätzlich zu vermeiden. Undeutliche abgebildete Kondylen sowie Anomalien, welche nicht eindeutig mit den Definitionen des genutzten Scoresystems zu vereinbaren waren, wurden daher von der Bewertung ausgeschlossen. Dennoch ist darauf hinzuweisen, dass eine gesicherte Aussage zur Kiefergelenkmitbeteiligung bei JIA-Patienten alleine durch Zuhilfenahme des in der Kiefergelenksdiagnostik als Goldstandard geltenden MRT getroffen werden kann (Carvalho et al. 2012, Müller et al. 2009, Weiss et al. 2008). Dies gilt insbesondere bei frühen Arthritisformen, wo Strukturanomalien im OPG noch nicht erkennbaren sind (Keller et al. 2015).

Bei jungen JIA-Patienten ist die Erfassung parodontaler Erkrankungen durch den PSI sowie die Erfassung auffälliger Kiefergelenksbefunde durch einen definierten 
Cut-off-Wert für die eingeschränkte MÖ, ebenfalls kritisch zu diskutieren. Bei der Beurteilung kindlicher Gebisse durch den PSI und somit durch den Parameter "Taschentiefe" könnten erhöhte Werte bei Kindern auch auf den Durchbruch von Zähnen im Wechselgebiss zurückzuführen sein (Nobre et al. 2016). Die „British Society of Periodontology“ und die "British Society of Paetiatric Dentistry“ proklamieren daher, dass bei Kindern von 7-11 Jahren nur die PSI-Codes 0, 1 und 2 genutzt werden, um eine Übertherapie von Pseudotaschen zu vermeiden (Heasman und Waterhouse 2012). In der klinischen Diagnostik der eingeschränkten MÖ wurde in der vorliegenden Untersuchung ein Cut-off-Wert von $39 \mathrm{~mm}$ benutzt. Dieser Wert wurde in Anlehnung an den beim Helkimo-Index verwendeten Wert sowie auf Grundlage vorangegangener Untersuchungen gewählt (Carvalho et al. 2012, Müller et al. 2009, Kitai et al. 2002, Helkimo 1974). Ein Problem stellt jedoch die große Varianz von Mundöffnungsdistanzen bei gesunden Kindern innerhalb einer Altersgruppe dar. Landtwing et al. (1978) berichten beispielsweise von einer beobachteten Varianz der Mundöffnungsbewegung bei 5-Jährigen von $35-55 \mathrm{~mm}$ und bei 18-Jährigen von 36-65 mm.

Die Frage, ob die in dieser Studie ermittelten oralen Erkrankungsparameter und Kiefergelenkerkrankungen gegenüber einer gesunden Patientengruppe erhöht sind, kann aufgrund einer fehlenden Kontrollgruppe nicht abschließend beantwortet werden. Ebenfalls muss bei der Beurteilung der hier durchgeführten Zusammenhangsuntersuchungen berücksichtigt werden, dass zahlreiche in dieser Studie nicht beachtete Parameter, wie beispielsweise die Medikamenteneinnahme, ebenfalls für die Erkrankung von Zahn, Parodont oder Kiefergelenken verantwortlich sein können (Fabri et al. 2014). Mit Blick auf die vorgenommenen statistischen Zusammenhangsanalysen ist die geringe Patientenzahl, gepaart mit einer nicht vorhandene Normalverteilung und einem zumeist geringen Skalenniveau der erhobenen Daten, zu nennen. Tiefergehende Analysen, bspw. der Einfluss möglicher Störgrößen (Alter, Geschlecht etc.) auf die univariat ermittelten Zusammenhänge oder eine gleichzeitige Betrachtung mehrerer Einflussvariablen auf eine interessierende Zielgröße, konnten aufgrund unzureichender Testvoraussetzungen nicht durchgeführt werden. Zugleich weisen die angewandten nichtparametrischen Tests im Vergleich zu parametrischen Verfahren eine relativ geringe Power auf. Die in dieser Arbeit beschriebenen Auffälligkeiten sind demnach nur als erste Hinweise zu interpretieren und bedürfen weitergehender Fall-Kontroll-Studien. 


\section{Schlussfolgerung}

Im Vergleich zur deutschen „Allgemeinbevölkerung“ (repräsentative Daten der DMS V) zeigten die hier untersuchten Kinder und Jugendlichen mit diagnostizierter JIA einen schlechteren Mundgesundheitszustand. Zudem waren erwartungsgemäß häufig auffällige Kiefergelenksbefunde festzustellen. Frühzeitige und regelmäßige Kontrollen des Mundgesundheitszustandes und der Kiefergelenke sind bei diesem Patientenklientel somit unerlässlich und sollten in der zahnärztlichen Praxis effizient durchgeführt werden können. Es sind weitere Untersuchungen mit größeren Patientengruppen notwendig, um auf Basis der festgestellten Ergebnisse mögliche Risikofaktoren für die orale und Kiefergelenksgesundheit bei JIA Patienten zu identifizieren. Bezogen auf die Kiefergelenkdiagnostik konnte in der vorliegenden Untersuchung kein Parameter identifiziert werden, welcher bei JIAPatienten zuverlässig auf eine Kiefergelenkbeteiligung bzw. -destruktion hinweist. Folglich sind die in dieser Arbeit ermittelten Ergebnisse zu anamnestischen und klinischen Diagnoseparametern der Kiefergelenksuntersuchung bei JIA-Patienten nur als erste Hinweise für eine Verbesserung dieser Diagnostik zu interpretieren. Die Notwendigkeit zur Erfassung geeigneter und zielgerichteter Parameter sowie deren Kombination zu Diagnostiksystemen bleibt bestehen. Dies sollte ebenfalls Bestandteil zukünftiger multivariater und - im optimalen Falle - MRT-basierter Studien mit größeren Patientengruppen sein. 


\section{Zusammenfassung}

Ziel dieser klinischen Querschnittsstudie war die Überprüfung der Mundgesundheit und auffälliger Kiefergelenksbefunde bei JIA-Patienten. Zusammenhänge zu Patienten- und Erkrankungscharakteristika sollten berücksichtigt werden. Zudem wurden Auffälligkeiten der Kiefergelenksdiagnostik aus Anamnese, klinischer Untersuchung und Röntgenauswertung miteinander verglichen.

59 Patienten (42 weiblich, 17 männlich) wurden aufgrund definierter Ein- und Ausschlusskriterien einbezogen. Aus Patientenakten und einem speziell entwickelten Anamnesebogen wurden erkrankungsbezogene Informationen zum Zeitpunkt der Diagnosestellung, Krankheitsverlauf, Medikation und Blutwerte entnommen. Über einen Funktionsfragebogen wurden Schmerzen und Funktionseinschränkungen im Mund-Kiefer-Gesichtsbereich erfragt. Bei der klinischen Untersuchung des oralen Zustandes wurden der dmf-t/DMF-T, der PBI und PSI erhoben. Die Kiefergelenkuntersuchung erfolgte nach den Vorgaben der RDC/TMD und der Auswertung vorliegender Röntgenbilder (OPG).

Der mittlere dmf-t/DMF-T betrug 2,6 $\pm 3,0$. Ein Patient wies den PBI 0 auf, bei $43 \%$ wurden stärkere Blutungen (PBI 3 oder 4) beobachtet. Nach der PSI-Auswertung waren drei Patienten parodontal gesund (PSI 0). 39\% Patienten wiesen Sondierungstiefen von 4-5mm (PSI 3) auf. Für die Erkrankungsparameter Erkrankungsform, Erkrankungsdauer, Beteiligung oberer Extremitäten, Röntgenscore, Schmerzen und eingeschränkte MÖ konnten keine signifikanten Einflüsse auf die Mundoder Kiefergelenksgesundheit festgestellt werden. Bei den Blutparametern wurden signifikante Zusammenhänge zwischen verstärkten gingivalen Blutungen bei ANAnegativen Patienten $(p=0,011)$ und Patienten mit erhöhten CRP-Werten $(p=0,034)$ ermittelt. Zudem war ein Zusammenhang erhöhter CRP-Werte mit steigenden Röntgenscores zu beobachten ( $p=0,043$ ). 47\% der Patienten zeigten strukturelle Auffälligkeiten der Kiefergelenke im OPG. $41 \%$ der Patienten waren in der anamnestischen, $52 \%$ in der klinischen Kiefergelenkdiagnostik auffällig. Lediglich für die eingeschränkte MÖ ergab sich eine signifikante Übereinstimmung von positiver Anamnese und klinischer Diagnostik $(p=0,001)$. Die positive Anamnese ging wie die positive klinische Diagnostik mit signifikant erhöhten Röntgenscores einher $(p=0,01$ bzw. $p=0,006$ ). Bei Patienten, die in mindestens einem der untersuchten Parameter 
der klinischen und/oder anamnestischen Diagnostik auffällig waren, wurden ausschließlich höhere Röntgenscores von 2 und 3 nachgewiesen ( $p=0,022)$.

Die Ergebnisse zeigen, dass frühzeitige und regelmäßige Kontrollen des Mundgesundheitszustandes und der Kiefergelenke bei diesem Patientenklientel unerlässlich sind. Die Notwendigkeit weiterer Untersuchungen zur Optimierung der Kiefergelenkdiagnostik und zur Ermittlung bedeutsamer Risikofaktoren für die orale und Kiefergelenksgesundheit bleibt bestehen. 


\section{Abstract}

The aim of this clinical cross-sectional study was to examine oral health and abnormal findings with regard to the temporomandibular joints in JIA patients. Connections to patient and disease characteristics should be considered. In addition, correlations between conspicuous parameters of temporomandibular joint diagnostics from anamnesis, clinical examination and $\mathrm{X}$-ray evaluation were to be determined.

59 patients (42 female, 17 male) were included on the basis of defined inclusion and exclusion criteria. From patient files and a specially developed anamnesis questionnaire, disease-related information on the time of diagnosis, course of disease, medication and blood values were taken. A functional questionnaire was used to ask for pain and functional limitations in the oral and maxillofacial region. In the clinical examination of the oral condition, the dmf-t/DMF-T, PBI and PSI were surveyed. The temporomandibular joint examination was performed according to RDC/TMD guidelines and the evaluation of available radiographs (OPG).

The mean $\mathrm{dmf}-\mathrm{t} / \mathrm{DMF}-\mathrm{T}$ was 2.6 \pm 3.0 . One patient had a PBI $0 ; 43 \%$ had more severe bleeding (PBI 3 or 4). After PSI evaluation, three patients were inconspicuous with regard to the periodontal health (PSI 0). 39\% of patients had probing depths of $4-5 \mathrm{~mm}$ (PSI 3). No significant effects on oral or temporomandibular joint health were observed for the following disease parameters: form of disease, duration of disease, involvement of upper joints, $x$-ray score, pain and limited mouth opening (MO). In the blood parameters significant correlations between increased gingival bleeding in ANA-negative patients $(p=0.011)$ and patients with elevated CRP values $(p=0.034)$ were observed. In addition, an association of elevated CRP values with increasing $X$-ray scores was observed $(p=0.043) .47 \%$ of the patients showed structural abnormalities of the temporomandibular joints in the OPG. $41 \%$ of the patients were conspicuous in anamnestic and $52 \%$ in clinical TMJ diagnostics. Only for the limited $\mathrm{MO}$ there was a significant agreement between positive anamnesis and clinical diagnosis $(p=0.001)$. Like the positive clinical diagnosis, the positive anamnesis was accompanied by significantly increased $X$-ray scores $(p=0.01$ and $p=0.006$, respectively). In patients who were conspicuous in at least one of the examined parameters of clinical and/or anamnestic diagnostics, only higher X-ray scores of 2 and 3 were found $(p=0.022)$. 
The results show that early and regular controls of the oral health condition and the mandibular joints are indispensable for this patient clientele. The need for further examinations to optimize temporomandibular joint diagnostics and to identify significant risk factors for oral and temporomandibular joint health remains. 


\section{Anhang}

\subsection{Anamnesebogen}

PD. Dr. med. dent. Dirk Ziebolz, Telefon: 0551/39-22877; Universitätsmedizin Göttingen, GeorgAugust-Universität; Zentrum Zahn,- Mund- und Kieferheilkunde, Poliklinik für Präventive Zahnmedizin, Parodontologie und Kariologie; Robert-Koch-Str. 40, 37075 Göttingen

\section{Anamnesebogen}

Klinische Querschnittsstudie zur Beurteilung der Mundgesundheit und möglicher Dysfunktionen des stomatognathen Systems bei Kindern und Jugendlichen mit Juveniler Idiopathischer Arthritis

Pat.- / Code-Nr.:

Datum:

Bitte beantworte(n) Sie die folgenden Fragen bzw. kreuzen Sie Zutreffendes an. Mehrfachantworten sind möglich. Die gewissenhafte Beantwortung ist eine Voraussetzung für den Erfolg der Studie!

1. Bist Du / sind Sie in ständiger ärztlicher Behandlung?

Ja nein

Wenn ja, weswegen?

2. Leidest Du / leiden Sie an einer Herzerkrankung? (z. B. A. pectoris, Endokarditis, Klappenfehler)

3. Musst Du / müssen Sie ständig Medikamente einnehmen?

Wenn ja, welche?

(z. B. zur Blutzuckersenkung; gegen Herzbeschwerden, Bluthochdruck; zur Hemmung der Blutgerinnung; Rheumamittel; Beruhigungs/Schlaftabletten)

4. Bist Du / sind Sie zur Zeit in ärztlicher Behandlung? Wenn ja, weswegen?

5. Wann bist Du / sind Sie zum letzten Mal zahnärztlich untersucht worden?

6. Sind Deine / Ihre Zähne temperaturempfindlich? 
7. Blutet Dein / Ihr Zahnfleisch?

[ ] [ ]

8. Bemerkst Du / bemerken Sie Stellungsveränderungen Ihrer Zähne?

9. Hast Du / haben Sie manchmal einen schlechten Geschmack im Mund?

10. Hast Du / haben Sie wegen Zahnlockerung bzw. Zahnfleischbeschwerden schon einmal einen Zahnarzt aufgesucht?

Wenn ja, was wurde gemacht?

11. Wurde bei Dir / Ihnen bereits eine "Parodontose"-Behandlung durchgeführt?

Wenn ja, wann?

12. Rauchst Du/ Rauchen Sie oder hast Du / haben Sie geraucht?

13. Seit welchem Lebensjahr rauchst/rauchen oder hast/haben Du / Sie geraucht?

14. Wie viele Zigaretten/ Schachteln pro Tag etwa? Zigaretten/ Tag, Schachteln/ Tag

15. Seit wie vielen Jahren rauchst/rauchen Du / Sie nicht mehr? 


\subsection{Fragebögen}

PD. Dr. med. dent. Dirk Ziebolz, Telefon: 0551/39-22877; Universitätsmedizin Göttingen, GeorgAugust-Universität; Zentrum Zahn,- Mund- und Kieferheilkunde, Poliklinik für Präventive Zahnmedizin, Parodontologie und Kariologie; Robert-Koch-Str. 40, 37075 Göttingen

\section{Spezifisch-anamnestische Angaben der Rheumapatienten}

„Klinische Querschnittsstudie zur Beurteilung der Mundgesundheit und möglicher Dysfunktionen des stomatognathen Systems bei Kindern und Jugendlichen mit Juveniler Idiopathischer Arthritis“

Pat.- / Code-Nr.:

Datum:

Bitte beantworte(n) Sie die folgenden Fragen bzw. kreuze Zutreffendes an. Die gewissenhafte Beantwortung ist eine Voraussetzung für den Erfolg der Studie!

Wann wurde die Diagnose „rheumatoide Arthritis“ das erste Mal gestellt?

War Dein / Ihr Unterkiefer jemals blockiert, oder hast Du / hatten Sie jemals Schwierigkeiten, den Mund vollständig zu öffnen?

$\square$ ja $\square$ nein

Wenn ja, war diese Mundöffnungsbehinderung so stark, dass dabei Deine / Ihre Fähigkeit zu essen beeinflusst war?

$\square$ ja $\square$ nein

Hast Du / haben Sie den Eindruck, jemals das Kauen harter Speisen vermieden zu haben?

$\square$ ja $\square$ nein

Knackt es in Deinem / Ihrem Kiefergelenk, wenn Du / Sie den Mund öffnest oder schließt oder wenn Du / Sie kaust/kauen?

$\square$ ja $\square$ nein

Nimmst Du / nehmen Sie in Deinem / Ihrem Kiefergelenk ein reibendes Geräusch wahr, wenn Du / Sieden Mund öffnest oder schließt oder wenn Du / Sie kaust/kauen?

$\square$ ja $\square$ nein

Wurde Dir / Ihnen gesagt oder hast Du / haben Sie selbst bemerkt, dass Du / Sie im Schlaf mit den Zähnen presst/pressen oder knirschst/knirschen?

$\square$ ja $\square$ nein 
Presst/Pressen oder knirschst/knirschen Du / Sie mit den Zähnen am Tage?

$\square$ ja $\square$ nein

Hast du / haben Sie Ohrgeräusche oder Ohrklingen?

$\square$ ja $\square$ nein

Fühlt sich der Zusammenbiss Deiner / Ihrer Zähne ungewöhnlich oder unbequem an?

$\square$ ja $\square$ nein 
Dr. med. dent. Dirk Ziebolz, Telefon: 0551/39-22877; Universitätsmedizin Göttingen, GeorgAugust-Universität; Zentrum Zahn,- Mund- und Kieferheilkunde, Poliklinik für Präventive Zahnmedizin, Parodontologie und Kariologie; Robert-Koch-Str. 40, 37075 Göttingen

\section{Schmerzbefragung der Rheumapatienten}

„Klinische Querschnittsstudie zur Beurteilung der Mundgesundheit und möglicher Dysfunktionen des stomatognathen Systems bei Kindern und Jugendlichen mit Juveniler Idiopathischer Arthritis“

Pat.- / Code-Nr.:

Datum:

Bitte beantworte(n) Sie die folgenden Fragen bzw. kreuze Zutreffendes an. Die gewissenhafte Beantwortung ist eine Voraussetzung für den Erfolg der Studie!

Hattest Du / haben Sie während der vergangenen 6 Monate Probleme mit Kopfschmerzen oder Migräne?

$\square$ ja $\square$ nein

Hattest oder hast Du / haben Sie Schmerzen im Bereich des Kiefers, der Kiefergelenke oder der Schläfen?

$\square$ ja $\square$ nein

Hast Du / haben Sie beim morgendlichen Erwachen Schmerzen im Kieferbereich oder das Gefühl einer eingeschränkten Unterkieferbeweglichkeit?

$\square$ ja $\square$ nein

Wenn bei Dir/ Ihnen keine dieser Beschwerden vorliegt, so müssen die weiteren Fragen nicht mehr beantwortet werden

Ist Dein / Ihr Gesichtsschmerz dauernd, wiederkehrend oder trat er nur einmalig auf?

Dauernd

Wiederkehrend

Nur einmalig

Wann begann der Gesichtsschmerz zum ersten Mal?

Bist Du / sind Sie aufgrund der Gesichtsschmerzen zu einem Arzt, Zahnarzt oder zu Angehörigen anderer Heilberufe gegangen?

Nein

Ja, innerhalb der letzten 6 Monate

Ja, vor mehr als 6 Monaten 
Hast Du / haben Sie in der letzten Zeit eine Verletzung/Unfall im Bereich des Kiefers oder des Gesichtes erlitten?

$\square$ ja $\square$ nein

Wenn ja, hattest Du / hatten Sie Deinen/Ihren Gesichtsschmerz schon vor diesem Ereignis?

$\square$ ja $\square$ nein

Wie intensiv war der stärkste Schmerz in den vergangenen 6 Monaten auf einer Skala von 0 "kein Schmerz" bis 10 "stärkster vorstellbarer Schmerz"?

$\begin{array}{llllllllllllll}0 & 1 & 2 & 3 & 4 & 5 & 6 & 7 & 8 & 9 & 10 & \text { (Kein Schmerz } & --- & \text { stärkster vorstellbarer Schmerz) }\end{array}$

Wie intensiv war der durchschnittliche Schmerz in den vergangenen 6 Monaten auf einer Skala von 0 "kein Schmerz" bis 10 "stärkster vorstellbarer Schmerz"? Gemeint sind Schmerzen die Du/ Sie gewöhnlich, also oft empfunden haben.

$\begin{array}{lllllllllll}0 & 1 & 2 & 3 & 4 & 5 & 6 & 7 & 8 & 9 & 10\end{array}$ (Kein Schmerz $\quad$--- $\quad$ stärkster vorstellbarer Schmerz) 


\subsection{Patientenaufklärungen}

PD. Dr. med. dent. Dirk Ziebolz, Telefon: 0551/39-22877; Universitätsmedizin Göttingen, GeorgAugust-Universität; Zentrum Zahn,- Mund- und Kieferheilkunde, Poliklinik für Präventive Zahnmedizin, Parodontologie und Kariologie; Robert-Koch-Str. 40, 37075 Göttingen

Patientenaufklärung - minderjährige Patienten von 6-11 Jahren

„Klinische Querschnittsstudie zur Beurteilung der Mundgesundheit und möglicher Dysfunktionen des stomatognathen Systems bei Kindern und Jugendlichen mit Juveniler Idiopathischer Arthritis

\section{Lieber Patient, liebe Patientin!}

Hiermit möchten wir Dich um die freiwillige Teilnahme an unserer Studie zur Mundgesundheit und Gesundheit der Kiefergelenke bei Kindern und Jugendlichen mit einer rheumatischen Gelenkerkrankung bitten.

Warum werden junge Patienten mit einer entzündlichen Gelenkerkrankung zahnärztlich untersucht?

Bei Dir wurde eine entzündliche Erkrankung der Gelenke festgestellt. Diese Erkrankung wird auch als rheumatische Erkrankung bezeichnet. Es konnte beobachtet werden, dass Kinder und Jugendliche mit solchen Erkrankungen, wie Du, auch Problemen im Mund haben. Dabei kann es zu Löchern im Zahn kommen (Karies) oder das Zahnfleisch blutet beim Zähneputzen; einige Kinder können ihren Mund nicht richtig öffnen und das Kiefergelenk tut weh.

Um zu verhindern, dass solche Probleme im Mund auftreten, ist es wichtig die Zähne, das Zahnfleisch und das Kiefergelenk regelmäßig vom Zahnarzt untersuchen zu lassen. So kann man frühzeitig Probleme erkennen und dir helfen, dass es nicht schlimmer wird.

Aus diesem Grund werden Patienten wie Du, während sie im Krankenhaus sind, in unserer Zahnklink von einem Zahnarzt untersucht. Die Ergebnisse dieser Untersuchung werden von dem Zahnarzt notiert.

\section{Ablauf der zahnärztlichen Untersuchung}

Die Untersuchung dauert ungefähr 30-45 Minuten. Mit einem Spiegel kontrolliert der Zahnarzt die Anzahl Deiner Zähne, zählt die Löcher und schaut ob das Zahnfleisch blutet. Für die Kontrolle des Zahnfleischs nimmt der Zahnarzt eine Sonde. Dadurch kann es bei dieser Untersuchung etwas pieken und das kann unangenehm sein, ganz selten blutet das Zahnfleisch danach. Zum Schluss werden vom Zahnarzt mit den Fingern noch Deine Kiefergelenke und Backenmuskeln abgetastet und kontrolliert wie weit Dein Mund aufgeht. Zur besseren Beurteilung Deiner Kiefergelenke muss ein Röntgenbild gemacht werden. 
Zusätzlich zu der Untersuchung möchten wir Dich bitten gemeinsam mit deinen Eltern einige Fragen zu beantworten. Dabei geht es zum Beispiel darum, ob schon einmal Schmerzen im Bereich der Kiefergelenke aufgetreten sind, oder ob Du schon einmal vom Zahnarzt oder Kieferorthopäden behandelt wurdest.

Neben diesem Zahnarztbesuch haben wir geplant, Dich in einigen Jahren noch einmal zu untersuchen um zu sehen, wie es dann in deinem Mund aussieht. Diese Untersuchung ist allerdings für Dich freiwillig und du musst nicht kommen, wenn Du nicht willst.

\section{Warum und wie werden die weiteren Untersuchungen für die Studie durchgeführt?}

Uns interessiert ob Deine Kiefergelenke, die Zähne und das Zahnfleisches gesund sind und wie es im Vergleich bei anderen Kindern mit Deiner Krankheit aussieht.

Unsere Untersuchung besteht aus zwei Teilen: A) Zunächst möchten wir uns die Untersuchungsergebnisse aus der ersten Untersuchung ansehen und auswerten. Zum anderen fragen wir auch Deinen Arzt Herrn Dr. Rühlmann nach Informationen zu Deiner Erkrankung. B) Später würden wir Dich nach einigen Jahren nochmals zu einer zahnärztlichen Kontrolluntersuchung einladen.

\section{Muss man an den weiteren Untersuchungen für die Studie teilnehmen?}

An dieser geplanten Untersuchung musst Du nicht teilnehmen. Die Teilnahme ist freiwillig. Auch zu einem späteren Zeitpunkt kannst Du Deine Teilnahme zu jeder Zeit absagen. Durch solch eine Absage hast Du keinerlei Nachteile.

\section{Werden Name und Untersuchungsergebnisse geheim gehalten?}

Dein Name und Deine persönlichen Daten (z. B. Dein Geburtstag) werden nicht weitergegeben und werden vertraulich behandelt. Das bedeutet, dass Dein Name nicht weiter genannt wird.

Deine Teilnahme an der Studie, A) Auswertung der Akte und B) spätere Untersuchung, ist freiwillig. Ohne Deine Einwilligung und die Einwilligung Deiner Eltern durch eine Unterschrift dürfen wir die Untersuchungsergebnisse und Daten nicht auswerten und keine Informationen von anderen Ärzten einholen.

Falls du noch Fragen oder etwas nicht verstanden hast, kannst du uns fragen, wir werden dir dann noch einmal alles in Ruhe erklären. 
PD. Dr. med. dent. Dirk Ziebolz, Telefon: 0551/39-22877; Universitätsmedizin Göttingen, GeorgAugust-Universität; Zentrum Zahn,- Mund- und Kieferheilkunde, Poliklinik für Präventive Zahnmedizin, Parodontologie und Kariologie; Robert-Koch-Str. 40, 37075 Göttingen

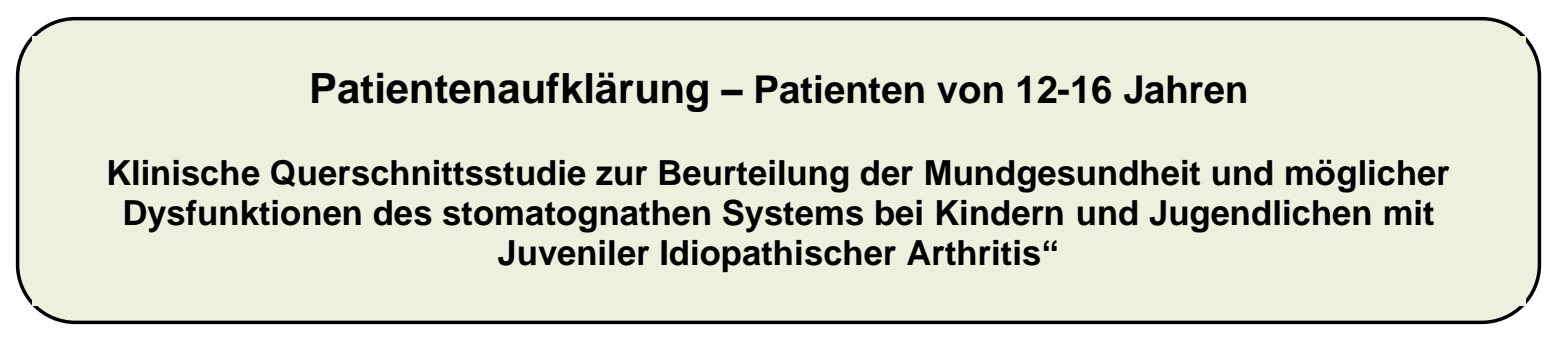

\section{Liebe Patientin, lieber Patient!}

Hiermit möchten wir Dich um die freiwillige Teilnahme an unserer Studie zur Mundgesundheit und Gesundheit der Kiefergelenke bei Kindern und Jugendlichen mit einer rheumatischen Gelenkerkrankung bitten.

\section{Warum werden junge Patienten mit einer entzündlichen Gelenkerkrankung zahnärztlich un- tersucht?}

Bei Dir wurde eine entzündliche Erkrankung der Gelenke festgestellt. Diese Erkrankung wird auch als rheumatische Erkrankung bezeichnet. Es konnte beobachtet werden, dass Kinder und Jugendliche mit solchen entzündlichen Gelenkerkrankungen des Öfteren unter Erkrankungen und Problemen im Mundraum leiden. Dabei kann es sich um Erkrankungen der Zähne handeln (Karies), es kann aber auch zu Entzündungen des Zahnfleisches (Gingivitis) kommen. Zudem leiden einige Kinder und Jugendliche an (schmerzhaften) Problemen bei der Mundöffnung oder der Kiefergelenke. Die Gründe und das Ausmaß dieser Probleme bleiben oftmals lange Zeit unbemerkt. Darum ist eine frühzeitige Untersuchung der Kiefergelenke aller Kinder und Jugendlichen mit einer entzündlichen Gelenkerkrankung sehr wichtig.

Aus diesem Grund werden junge Rheumapatienten wie Du, während sie im Krankenhaus sind, in unserer Zahnklink von einem Zahnarzt standardmäßig untersucht. Die Ergebnisse dieser Untersuchung werden von dem Zahnarzt notiert.

\section{Ablauf der zahnärztlichen Untersuchung}

Die Untersuchung dauert ungefähr 30-45 Minuten. Mit einem zahnärztlichen Spiegel kontrolliert der Zahnarzt die Anzahl Deiner Zähne und untersucht ob die Zähne Karies haben. Für die Überprüfung Deines Zahnfleisches benutzt der Zahnarzt den zahnärztlichen Spiegel und eine zahnärztliche Tastsonde. Diese Untersuchung kann etwas pieken und dadurch unangenehm sein, gelegentlich kann es zu Zahnfleischbluten kommen. Abschließend werden vom Zahnarzt Deine Kiefergelenk und Deine Kaumuskulatur abgetastet und die Beweglichkeit Deines Unterkiefers kontrolliert. Die Untersuchung der Kiefergelenke und der Kaumuskulatur kann für einige Patienten ebenfalls unangenehm sein und gegebenenfalls wehtun. Zur besseren Beurteilung Deiner Kiefergelenke müssen zusätzlich Röntgenbilder von Deinen Kiefergelenken gemacht werden. 
Zusätzlich zu der Untersuchung möchten wir Dich bitten gemeinsam mit deinen Eltern einige Fragen zu beantworten. Dabei geht es zum Beispiel darum, ob schon einmal Schmerzen im Bereich der Kiefergelenke aufgetreten sind, oder ob Du schon einmal vom Zahnarzt oder Kieferorthopäden behandelt wurdest.

Neben diesem Zahnarztbesuch haben wir geplant Dich in einigen Jahren noch einmal zu untersuchen um zu sehen, wie es dann in deinem Mund aussieht. Diese Untersuchung ist allerdings für Dich freiwillig und du musst nicht kommen, wenn Du nicht willst. Bei dieser erneuten Untersuchung werden keine studienbedingten Röntgenbilder angefertigt.

Warum und wie werden die weiteren Untersuchungen für die Studie durchgeführt?

Uns interessiert ob Deine Kiefergelenke, die Zähne und das Zahnfleisch gesund sind und wie es im Vergleich bei anderen Kindern mit Deiner Krankheit aussieht.

Unsere Untersuchung besteht aus zwei Teilen: A) Zunächst möchten wir uns die Untersuchungsergebnisse aus der ersten Untersuchung ansehen und auswerten. Zum anderen fragen wir auch Deinen Arzt Herrn Dr. RühImann nach Informationen zu Deiner Erkrankung. B) Später würden wir Dich nach einigen Jahren nochmals zu einer zahnärztlichen Kontrolluntersuchung einladen.

\section{Muss man an den weiteren Untersuchungen für die Studie teilnehmen?}

An dieser geplanten Untersuchung musst Du nicht teilnehmen. Die Teilnahme ist freiwillig. Auch zu einem späteren Zeitpunkt kannst Du Deine Teilnahme zu jeder Zeit absagen. Durch solch eine Absage hast Du keinerlei Nachteile.

\section{Werden Name und Untersuchungsergebnisse geheim gehalten?}

Dein Name und Deine persönlichen Daten (z. B. Dein Geburtstag) werden nicht weitergegeben und werden vertraulich behandelt. Das bedeutet, dass Dein Name nicht weiter genannt wird.

Deine Teilnahme an der Studie, A) Auswertung der Akte und B) spätere Untersuchung, ist freiwillig. Ohne Deine Einwilligung und die Einwilligung Deiner Eltern durch eine Unterschrift dürfen wir die Untersuchungsergebnisse und Daten nicht auswerten und keine Informationen von anderen Ärzten einholen.

Falls du noch Fragen oder etwas nicht verstanden hast, kannst du uns fragen, wir werden dir dann noch einmal alles in Ruhe erklären. 
PD. Dr. med. dent. Dirk Ziebolz, Telefon: 0551/39-22877; Universitätsmedizin Göttingen, GeorgAugust-Universität; Zentrum Zahn,- Mund- und Kieferheilkunde, Poliklinik für Präventive Zahnmedizin, Parodontologie und Kariologie; Robert-Koch-Str. 40, 37075 Göttingen

\section{Patientenaufklärung - Patienten ab 16 Jahren}

Klinische Querschnittsstudie zur Beurteilung der Mundgesundheit und möglicher Dysfunktionen des stomatognathen Systems bei Kindern und Jugendlichen mit Juveniler Idiopathischer Arthritis“

\section{Liebe Patientin, lieber Patient!}

Hiermit möchten wir Dich / Sie um die freiwillige Teilnahme an der klinisch-wissenschaftlichen Studie zur Mundgesundheitssituation und Gesundheit der Kiefergelenke bei Kindern und Jugendlichen mit Juveniler idiopathischer Arthritis bitten.

\section{Hintergrund}

Bei Dir / Ihnen wurde eine chronische, entzündliche Erkrankung der Gelenke (Arthritis) unbekannter Ursache (idiopathisch) diagnostiziert. Diese Erkrankung nennt man heute Juvenile Idiopathische Arthritis (abgekürzt JIA, früher: Juvenile rheumatoide Arthritis, Juvenile chronische Arthritis). Unter den verschiedenen ärztlichen Disziplinen wird immer mehr Wert darauf gelegt, herauszufinden, in welcher Art Zusammenhänge zwischen allgemeinen Erkrankungen und Erkrankungen der Mundhöhle, wie Karies (Erkrankung der Zähne), Gingivitis (Erkrankung des Zahnfleisches) oder Parodontitis (Erkrankung des Zahnhalteapparates) bestehen.

Bei JIA-Patienten konnte ein verstärktes Vorkommen oraler Erkrankungen, wie beispielsweise Karies beobachtet werden. Ein Grund hierfür könnten Funktionseinschränkungen sein, unter welchen Patienten mit einer JIA leiden und die eine adäquate Mundhygiene erschweren. Hier wäre insbesondere die eingeschränkte Mundöffnung bei Kiefergelenksbefall (entzündliche Mitbeteiligung der Kiefergelenke an der rheumatischen Erkrankung) und feinmotorische Einschränkungen bei Mitbeteiligung von Finger- und Handgelenken zu nennen.

Auch wenn vereinzelte Studien darauf hinweisen, so fehlen bis heute sichere Daten, ob ein klinischer Zusammenhang zwischen der JIA und entzündlichen Erkrankungen des Zahnhalteapparates (Gingivitis, Parodontitis) besteht. Allerdings gilt eine mögliche Mitbeteiligung der Kiefergelenke an der rheumatischen Grunderkrankung als gesichert. Es wird beschrieben, dass es bei bis zu $87 \%$ der Patienten zu einer Mitbeteiligung der Kiefergelenke kommt und dass in ca. 50\% der Fälle Symptome wie Schmerzen oder eine eingeschränkte Mundöffnung beobachtet werden können. Die Deutsche Rheumaliga weist darauf hin, dass die Untersuchung der Kiefergelenke aller Kinder und Jugendlichen mit JIA von großer Bedeutung ist.

Aufgrund der häufig auftretenden Kiefergelenksentzündungen bei der Juvenilen Idiopathischen Arthritis und der bereits erwähnten Zusammenhänge zu Erkrankungen im Mundraum werden (A) 
die stationären Rheumapatienten, der Klinik Pädiatrie II mit Schwerpunkt Neuropädiatrie der Universitätsmedizin Göttingen, im Rahmen des Klinikaufenthaltes grundsätzlich einer routinemäßigen zahnärztlichen Untersuchung der Zähne, des Zahnhalteapparates, sowie des stomatognathen Systems (Kiefergelenk, Kaumuskulatur) unterzogen.

Bei Patienten der kinderrheumatologischen Praxis Dr. Rühlmann (B) entscheidet der behandelnde Arzt individuell, welche Patienten er zu einer zahnärztlichen Untersuchung in unsere Klinik überweist.

\section{Ziel der Studie}

Ziel der Studie ist die Überprüfung der Mund- und Kiefergelenksgesundheit bei Kindern und Jugendlichen mit der Diagnose „Juvenile Idiopathische Arthritis“; zur Mundgesundheit zählen sowohl der Zustand der Zähne als auch von Zahnfleisch und Kieferknochen (Zahnhalteapparat) sowie der Kiefergelenke und der Kaumuskulatur.

Die geplante Untersuchung setzt sich aus zwei Studien zusammen:

A) Querschnittstudie: Auswertung vorliegender Patientenunterlagen aus den zahnärztlichen Kontrolluntersuchungen

B) Verlaufsstudie: zahnärztliche Kontrolluntersuchung nach einer Beobachtungszeit von 3-5 bzw. 10-12 Jahren

\section{Ablauf der zahnärztlichen Untersuchung}

Um Aussagen über Deine / Ihre vorliegende Mund- und Kiefergelenksgesundheit treffen zu können, werden innerhalb einer routinemäßigen zahnärztlichen Untersuchung (Konsilüberweisung) spezielle Befunde erhoben, die sich aus einem zahnärztlichen Befund (Erhebung von Anzahl der Zähne, Füllungen und Karies), der Messung der Zahnfleischentzündung (Blutung) und der Feststellung des Zustands des Zahnhalteapparates (Tiefenmessung des Bereiches zwischen Zahn und Zahnfleisch und Beurteilung evtl. auftretender Blutungspunkte) sowie einer Untersuchung des Kiefergelenks und der Kaumuskulatur (Funktionsstatus) zusammensetzen. Bei der klinischen Untersuchung des Kiefergelenks als auch der Kau- und Nackenmuskulatur werden diese abgetastet und die Beweglichkeit (Mobilität) des Unterkiefers ermittelt. Zudem werden Röntgenbilder zur Beurteilung der Kiefergelenke angefertigt.

Zudem bitten wir Dich / Sie, einige Fragen zum Allgemeinzustand, Lebensgewohnheiten und zahnärztlichem Verhalten sowie zu gegebenenfalls bestehenden Kiefergelenk- und/oder Muskelschmerzen zu beantworten. Bitte versuchen Sie diese Fragen wahrheitsgemäß und möglichst genau zu beantworten; ggf. werden vorliegende Röntgenbilder für die Auswertung berücksichtigt.

Die Befragung und Untersuchungen an den Zähnen und am Zahnfleisch führt ein Zahnarzt der Poliklinik für Präventive Zahnmedizin, Parodontologie und Kariologie der Universitätsmedizin Göttingen durch. Die zahnärztliche Untersuchung erfolgt während Deines/ Ihres Klinikaufenthaltes oder nach einem Untersuchungstermin in der kinderrheumatologischen Praxis in der Zahnklinik Göttingen (Poliklinik für Präventive Zahnmedizin, Parodontologie und Kariologie). Der Mehrauf- 
wand an Zeit pro Untersuchung beträgt ca. 30-45 Minuten für Patienten während des Klinikaufenthaltes und 60-90 Minuten für Patienten aus der Praxis; es entstehen für Dich / Sie keine zusätzlichen Kosten.

Risiken und Nebenwirkungen bei der Durchführung der Untersuchung sind nicht zu erwarten bzw. bestehen nicht, da keine Medikamente, operative Eingriffe oder Anfertigungen von Röntgenaufnahmen zu Studienzwecken notwendig sind; jedoch kann die Untersuchung sowohl der Zähne, des Zahnfleisches, als auch der Kiefergelenke und Kaumuskulatur ggf. unangenehm sein. Bei der Kontrolle des Zahnfleisches können möglicherweise geringfügige Blutungen provoziert werden.

Entsprechend der oben beschrieben routinemäßigen zahnärztlichen Untersuchung ist folgendes Vorgehen im Rahmen der Studie A) und B) vorgesehen:

A) Querschnittsstudie: Für unsere Studie wollen wir die bei der zahnärztlichen Untersuchung ermittelten Befunde, Daten und Fragebögen auswerten. Zudem benötigen wir vom Rheumatologen eine Auskunft über Zeitpunkt der Diagnosestellung der Juvenilen Idiopathischen Arthritis, Stadium der Erkrankung, Medikation und über ggf. vorhandene Blutparameter, wie z. B. Rheumafaktoren.

Hierfür bitten wir Dich / Sie um Einwilligung in die Datenauswertung.

B) Nach 3-5 bzw. 10-12 Jahren werden wir uns erneut an Dich / Sie wenden, um Dich / Sie zu einer Nachuntersuchung nach oben beschriebenem Muster einzuladen, um den Verlauf zu dokumentieren und auszuwerten. Allerdings werden keine studienbedingten/neuen Röntgenbilder mehr angefertigt. Diese Nachuntersuchung ist freiwillig und kostenfrei.

\section{Wiederruf}

Wir bitten um die freiwillige Teilnahme an der Studie. Du kannst / Sie können jederzeit die Teilnahme widerrufen, ohne Angabe von Gründen und ohne Nachteile erwarten zu müssen. Nach Deinem / Ihrem Wiederruf hast Du / haben Sie die Möglichkeit zum einen die unverzügliche Vernichtung Deiner/ Ihrer personenbezogenen Daten zu bewirken oder die Genehmigung der Auswertung der erhobenen Daten zu erteilen. Zudem entstehen Dir / Ihnen keine Nachteile durch eine Ablehnung der Teilnahme an der Untersuchung.

\section{Ausschluss}

Wenn Dir/ Ihnen bekannt ist, dass Du/ Sie eine infektiöse Gelbsucht (Hepatitis A, B oder C) oder eine Tuberkulose oder eine HIV-Infektion hast/ haben, bitten wir Dich/Sie dringend, uns das vertraulich mitzuteilen. Du/ Sie darfst/ dürfen in diesem Fall an der vorliegenden Studie nicht teilnehmen.

\section{Datenschutz}

Die personenbezogenen Daten unterliegen dem Datenschutz und werden vom Leiter der Prüfung nicht weitergegeben. Sie werden pseudonymisiert behandelt, d.h. Verschlüsselung von Daten ohne Namensnennung und Nummerncodierung. Die Zuordnung der Daten zu einer Person ist nur mög- 
lich, wenn hierfür der Schlüssel eingesetzt wird, mit dem die Daten pseudonymisiert wurden. Die personenbezogenen Daten werden unter besonderen Schutzvorkehrungen getrennt von den pseudonymisierten Daten aufbewahrt. Eine Entschlüsselung ist nur durch die verantwortlichen Studienärzte möglich; Dritte erhalten keinen Einblick in die Originalunterlagen. Im Rahmen der Studie werden die personenbezogenen Daten mit studienspezifischen Erfassungsbögen erhoben und pseudonymisiert in eine Exceltabelle übertragen. Auf die Daten haben nur der Leiter der Prüfung und die Prüfärzte Zugriff; die Daten sind durch ein Passwort gesichert. Die Prüfbögen (Erfassungsbögen) werden in einem Prüfordner gesammelt und mit den gespeicherten Daten beim Leiter der Prüfung für 10 Jahre aufbewahrt. Die gespeicherten Daten werden nur zu Untersuchungszwecken verwendet.

Die Teilnahme an der Studie ist freiwillig. Ohne Deine / Ihre Einwilligung und die Einwilligung Deiner / Ihrer Eltern durch eine schriftliche Einverständniserklärung als sorgeberechtigte Person dürfen wir die Untersuchungsergebnisse und Daten nicht auswerten und keine Informationen von Deinem/lhrem behandelndem Rheumatologen einholen.

Für Rückfragen stehen Ihnen der Studienleiter und der durchführende Zahnarzt unter o.g. Telefonnummer gerne zur Verfügung. 
PD. Dr. med. dent. Dirk Ziebolz, Telefon: 0551/39-22877; Universitätsmedizin Göttingen, GeorgAugust-Universität; Zentrum Zahn,- Mund- und Kieferheilkunde, Poliklinik für Präventive Zahnmedizin, Parodontologie und Kariologie; Robert-Koch-Str. 40, 37075 Göttingen

\section{Patientenaufklärung - Sorgeberechtigte}

„Klinische Querschnittsstudie zur Beurteilung der Mundgesundheit und möglicher Dysfunktionen des stomatognathen Systems bei Kindern und Jugendlichen mit Juveniler Idiopathischer Arthritis"

\section{Sehr geehrte Eltern, sehr geehrte Sorgeberechtigte!}

Hiermit möchten wir Sie um die freiwillige Teilnahme Ihres Kindes an der klinisch-wissenschaftlichen Studie zur Mundgesundheitssituation und Gesundheit der Kiefergelenke bei Kindern und Jugendlichen mit Juveniler idiopathischer Arthritis bitten.

\section{Hintergrund}

Bei Ihrem Kind wurde eine chronische, entzündliche Erkrankung der Gelenke (Arthritis) unbekannter Ursache (idiopathisch) diagnostiziert. Diese Erkrankung nennt man heute Juvenile Idiopathische Arthritis (abgekürzt JIA, früher: Juvenile rheumatoide Arthritis, Juvenile chronische Arthritis). Unter den verschiedenen ärztlichen Disziplinen wird immer mehr Wert darauf gelegt, herauszufinden, in welcher Art Zusammenhänge zwischen allgemeinen Erkrankungen und Erkrankungen der Mundhöhle, wie Karies (Erkrankung der Zähne), Gingivitis (Erkrankung des Zahnfleisches) oder Parodontitis (Erkrankung des Zahnhalteapparates) bestehen.

Bei JIA-Patienten konnte ein verstärktes Vorkommen oraler Erkrankungen, wie beispielsweise Karies beobachtet werden. Ein Grund hierfür könnten Funktionseinschränkungen sein, unter welchen Patienten mit einer JIA leiden und die eine adäquate Mundhygiene erschweren. Hier wäre insbesondere die eingeschränkte Mundöffnung bei Kiefergelenksbefall (entzündliche Mitbeteiligung der Kiefergelenke an der rheumatischen Erkrankung) und feinmotorische Einschränkungen bei Mitbeteiligung von Finger- und Handgelenken zu nennen.

Auch wenn vereinzelte Studien darauf hinweisen, so fehlen bis heute sichere Daten, ob ein klinischer Zusammenhang zwischen der JIA und entzündlichen Erkrankungen des Zahnhalteapparates (Gingivitis, Parodontitis) besteht. Allerdings gilt eine mögliche Mitbeteiligung der Kiefergelenke an der rheumatischen Grunderkrankung als gesichert. Es wird beschrieben, dass es bei bis zu $87 \%$ der Patienten zu einer Mitbeteiligung der Kiefergelenke kommt und dass in ca. 50\% der Fälle Symptome wie Schmerzen oder eine eingeschränkte Mundöffnung beobachtet werden können. Die Deutsche Rheumaliga weist darauf hin, dass die Untersuchung der Kiefergelenke aller Kinder und Jugendlichen mit JIA von großer Bedeutung ist. 
Aufgrund der häufig auftretenden Kiefergelenksentzündungen bei der Juvenilen Idiopathischen Arthritis und der bereits erwähnten Zusammenhänge zu Erkrankungen im Mundraum werden (A) die stationären Rheumapatienten, der Klinik Pädiatrie II mit Schwerpunkt Neuropädiatrie der Universitätsmedizin Göttingen, im Rahmen des Klinikaufenthaltes grundsätzlich einer routinemäßigen zahnärztlichen Untersuchung der Zähne, des Zahnhalteapparates, sowie des stomatognathen Systems (Kiefergelenk, Kaumuskulatur) unterzogen.

Bei Patienten der kinderrheumatologischen Praxis Dr. Rühlmann (B) entscheidet der behandelnde Arzt individuell, welche Patienten er zu einer zahnärztlichen Untersuchung in unsere Klinik überweist.

\section{Ziel der Studie}

Ziel der Studie ist die Überprüfung der Mund- und Kiefergelenksgesundheit bei Kindern und Jugendlichen mit der Diagnose „Juvenile Idiopathische Arthritis“; zur Mundgesundheit zählen sowohl der Zustand der Zähne als auch von Zahnfleisch und Kieferknochen (Zahnhalteapparat) sowie der Kiefergelenke und der Kaumuskulatur.

Die geplante Untersuchung setzt sich aus zwei Studien zusammen:

A) Querschnittstudie: Auswertung vorliegender Patientenunterlagen aus den zahnärztlichen Kontrolluntersuchungen

B) Verlaufsstudie: zahnärztliche Kontrolluntersuchung nach einer Beobachtungszeit von 3-5 bzw. 10-12 Jahren

\section{Ablauf der zahnärztlichen Untersuchung}

Um Aussagen über die vorliegende Mund- und Kiefergelenksgesundheit Ihres Kindes treffen zu können, werden innerhalb einer routinemäßigen zahnärztlichen Untersuchung (Konsilüberweisung) spezielle Befunde erhoben, die sich aus einem zahnärztlichen Befund (Erhebung von Anzahl der Zähne, Füllungen und Karies), der Messung der Zahnfleischentzündung (Blutung) und der Feststellung des Zustands des Zahnhalteapparates (Tiefenmessung des Bereiches zwischen Zahn und Zahnfleisch und Beurteilung evtl. auftretender Blutungspunkte) sowie einer Untersuchung der Kiefergelenke und der Kaumuskulatur (Funktionsstatus) zusammensetzen. Bei der klinischen Untersuchung der Kiefergelenke als auch der Kau- und Nackenmuskulatur werden diese abgetastet und die Beweglichkeit (Mobilität) des Unterkiefers ermittelt. Zudem werden Röntgenbilder zur Beurteilung der Kiefergelenke angefertigt.

Zudem bitten wir Sie, einige Fragen zum Allgemeinzustand, Lebensgewohnheiten und zahnärztlichem Verhalten sowie zu gegebenenfalls bestehenden Kiefergelenk- und/oder Muskelschmerzen Ihres Kindes zu beantworten. Bitte versuchen Sie diese Fragen wahrheitsgemäß und möglichst genau zu beantworten; ggf. werden vorliegende Röntgenbilder für die Auswertung berücksichtigt.

Die Befragung und Untersuchungen an den Zähnen und am Zahnfleisch führt ein Zahnarzt der Poliklinik für Präventive Zahnmedizin, Parodontologie und Kariologie der Universitätsmedizin Göttingen durch. Die zahnärztliche Untersuchung erfolgt während des Klinikaufenthaltes Ihres Kindes 
oder nach einem Untersuchungstermin in der kinderrheumatologischen Praxis in der Zahnklinik Göttingen (Poliklinik für Präventive Zahnmedizin, Parodontologie und Kariologie). Der Mehraufwand an Zeit pro Untersuchung beträgt ca. 30-45 Minuten für Patienten während des Klinikaufenthaltes und 60-90 Minuten für Patienten aus der Praxis; es entstehen für Sie keine zusätzlichen Kosten.

Risiken und Nebenwirkungen bei der Durchführung der Untersuchung sind nicht zu erwarten bzw. bestehen nicht, da keine Medikamente, operative Eingriffe oder Anfertigungen von Röntgenaufnahmen zu Studienzwecken notwendig sind; jedoch kann die Untersuchung sowohl der Zähne, des Zahnfleisches, als auch der Kiefergelenke und Kaumuskulatur ggf. unangenehm sein. Bei der Kontrolle des Zahnfleisches können möglicherweise geringfügige Blutungen provoziert werden.

Entsprechend der oben beschriebenen routinemäßigen zahnärztlichen Untersuchung ist folgendes Vorgehen im Rahmen der Studie A) und B) vorgesehen:

A) Querschnittsstudie: Für unsere Studie wollen wir die bei der zahnärztlichen Untersuchung ermittelten Befunde, Daten und Fragebögen Ihres Kindes auswerten. Zudem benötigen wir vom Rheumatolo-gen eine Auskunft über Zeitpunkt der Diagnosestellung der Juvenilen Idiopathischen Arthritis, Stadium der Erkrankung, Medikation und über ggf. vorhandene Blutparameter, wie z. B. Rheumafaktoren.

Hierfür bitten wir Sie um Einwilligung in die Datenauswertung.

B) Nach 3-5 bzw. 10-12 Jahren werden wir uns erneut an Sie wenden, um Ihr Kind zu einer Nachuntersuchung nach oben beschriebenem Muster einzuladen, um den Verlauf zu dokumentieren und auszuwerten. Allerdings werden keine studienbedingten/neuen Röntgenbilder mehr angefertigt. Diese Nachuntersuchung ist freiwillig und kostenfrei.

\section{Wiederruf}

Wir bitten um die freiwillige Teilnahme an der Studie. Sie können jederzeit die Teilnahme widerrufen, ohne Angabe von Gründen und ohne Nachteile erwarten zu müssen. Nach Ihrem Wiederruf haben Sie die Möglichkeit zum einen die unverzügliche Vernichtung Ihrer personenbezogenen Daten zu bewirken oder die Genehmigung der Auswertung der erhobenen Daten zu erteilen. Zudem entstehen Ihnen keine Nachteile durch eine Ablehnung der Teilnahme an der Untersuchung.

\section{Ausschluss}

Wenn Ihnen bekannt ist, dass Ihr Kind eine infektiöse Gelbsucht (Hepatitis A, B oder C) oder eine Tuberkulose oder eine HIV-Infektion hat, bitten wir Sie dringend, uns das vertraulich mitzuteilen. Ihr Kind darf in diesem Fall an der vorliegenden Studie nicht teilnehmen.

\section{Datenschutz}

Die personenbezogenen Daten unterliegen dem Datenschutz und werden vom Leiter der Prüfung nicht weitergegeben. Sie werden pseudonymisiert behandelt, d.h. Verschlüsselung von Daten ohne Namensnennung und Nummerncodierung. Die Zuordnung der Daten zu einer Person ist nur mög- 
lich, wenn hierfür der Schlüssel eingesetzt wird, mit dem die Daten pseudonymisiert wurden. Die personenbezogenen Daten werden unter besonderen Schutzvorkehrungen getrennt von den pseudonymisierten Daten aufbewahrt. Eine Entschlüsselung ist nur durch die verantwortlichen Studienärzte möglich; Dritte erhalten keinen Einblick in die Originalunterlagen. Im Rahmen der Studie werden die personenbezogenen Daten Ihres Kindes mit studienspezifischen Erfassungsbögen erhoben und pseudonymisiert in eine Exceltabelle übertragen. Auf die Daten haben nur der Leiter der Prüfung und die Prüfärzte Zugriff; die Daten sind durch ein Passwort gesichert. Die Prüfbögen (Erfassungsbögen) werden in einem Prüfordner gesammelt und mit den gespeicherten Daten beim Leiter der Prüfung für 10 Jahre aufbewahrt. Die gespeicherten Daten werden nur zu Untersuchungszwecken verwendet.

Die Teilnahme an der Studie ist freiwillig. Ohne die Einwilligung Ihres Kindes und Ihrer schriftlichen Einverständniserklärung als sorgeberechtigte Person dürfen wir die Untersuchungsergebnisse und Daten nicht auswerten und keine Informationen von dem Ihr Kind behandelndem Rheumatologen einholen.

Für Rückfragen stehen Ihnen der Studienleiter und der durchführende Zahnarzt unter o.g. Telefonnummer gerne zur Verfügung. 


\subsection{Einverständniserklärungen}

PD. Dr. med. dent. Dirk Ziebolz, Telefon: 0551/39-22877; Universitätsmedizin Göttingen, GeorgAugust-Universität; Zentrum Zahn,- Mund- und Kieferheilkunde, Poliklinik für Präventive Zahnmedizin, Parodontologie und Kariologie; Robert-Koch-Str. 40, 37075 Göttingen

\section{Einverständniserklärung (Patienten von 12-16 Jahren)}

„Klinische Querschnittsstudie zur Beurteilung der Mundgesundheit und möglicher Dysfunktionen des stomatognathen Systems bei Kindern und Jugendlichen mit Juveniler Idiopathischer Arthritis"

Ich (Patient), wurde von einem Arzt vollständig über Risiken, Wesen, Bedeutung und Tragweite sowie Durchführung der Studie (zum Zwecke der Forschung) mit dem Titel: „Klinische Querschnittsstudie zur Beurteilung der Mundgesundheit und möglicher Dysfunktionen des stomatognathen Systems bei Kindern und Jugendlichen mit Juveniler Idiopathischer Arthritis" aufgeklärt.

Mir ist bekannt, dass für die Untersuchungen persönliche Daten ausgewertet werden sollen. Die Daten werden verschlüsselt, also ohne dass mein Name genannt wird, notiert und gespeichert. Die Daten werden 10 Jahre lang aufbewahrt und danach gelöscht. Meine persönlichen Daten werden nicht an andere Personen weitergegeben.

Ich hatte ausreichend Zeit, mich zur Teilnahme an den Untersuchungen zu entscheiden und weiß, dass die Teilnahme freiwillig ist. Alle Fragen wurden zu meiner Zufriedenheit beantwortet.

Ich weiß, dass ich ohne einen Nachteil zu haben, jederzeit und ohne Angabe von Gründen meine Teilnahme wieder absagen kann. Ich bin damit einverstanden, dass meine persönlichen Daten auch nach meinem Widerruf weiter für die Auswertung verwendet werden dürfen.

ja $\square$ nein

Ich stimme zu, dass meine persönlichen Daten, Untersuchungsergebnisse und Röntgenbilder aus der zahnärztlichen Untersuchung ausgewertet werden dürfen

ja $\square$ nein $\square$

Ich bin damit einverstanden nach ein paar Jahren nochmals zu einer Kontrolluntersuchung eingeladen zu werden.

ja $\square$ nein $\square$

Für Praxispatienten: Ich entbinde meinen behandelnden Arzt (Dr. Rühlmann) von der Schweigepflicht gegenüber den Studienärzten der Poliklinik für Präventive Zahnmedizin, Parodontologie und Kariologie der Universitätsmedizin Göttingen. 
ja $\square$ nein $\square$

Ich möchte die in der Studie festgestellten Untersuchungsergebnisse mitgeteilt bekommen?

ja $\square$ nein $\square$

Ich habe eine Kopie der Patienteninformation und dieser Einwilligungserklärung erhalten und bin mit meiner freiwilligen Teilnahme an den zusätzlichen Untersuchungen einverstanden.

Ort und Datum

Unterschrift des Teilnehmers

Ort und Datum

Unterschrift des Prüfarztes 
PD. Dr. med. dent. Dirk Ziebolz, Telefon: 0551/39-22877; Universitätsmedizin Göttingen, GeorgAugust-Universität; Zentrum Zahn,- Mund- und Kieferheilkunde, Poliklinik für Präventive Zahnmedizin, Parodontologie und Kariologie; Robert-Koch-Str. 40, 37075 Göttingen

\section{Einverständniserklärung (Patienten ab 16 Jahren)}

„Klinische Querschnittsstudie zur Beurteilung der Mundgesundheit und mögliche Dysfunktionen des stomatognathen Systems bei Kindern und Jugendlichen mit Juveniler Idiopathischer Arthritis"

Ich (Patient), wurde von einem Arzt vollständig über Risiken, Wesen, Bedeutung und Tragweite sowie Durchführung der Studie (zum Zwecke der Forschung) mit dem Titel: „Klinische Querschnittsstudie zur Beurteilung der Mundgesundheit und möglicher Dysfunktionen des stomatognathen Systems bei Kindern und Jugendlichen mit Juveniler Idiopathischer Arthritis“ aufgeklärt.

Mir ist bekannt, dass im Rahmen dieses Forschungsvorhabens personenbezogene Daten ausgewertet werden sollen. Die Daten werden in pseudonymisierter (verschlüsselter) Form aufgezeichnet und gespeichert. Die Daten werden für einen Zeitraum von 10 Jahren aufbewahrt, danach werden alle personenbezogenen Daten gelöscht. Die personenbezogenen Daten werden nicht an Dritte weitergegeben.

Ich weiß, dass ich mein Einverständnis zur Teilnahme an der Studie jederzeit widerrufen kann. Im Falle des Widerrufs werden alle personenbezogenen Daten gelöscht.

Ich hatte ausreichend Zeit, mich zur Teilnahme meines Kindes an dieser Studie zu entscheiden und weiß, dass die Teilnahme freiwillig ist. Alle Fragen wurden zu meiner Zufriedenheit beantwortet.

Mir ist bekannt, dass ich jederzeit und ohne Angaben von Gründen diese Zustimmung widerrufen kann, ohne dass sich dieser Entschluss nachteilig auf meine weitere Behandlung auswirkt. Ich bin damit einverstanden, dass meine personenbezogenen Daten auch nach meinem Widerruf weiter für die Auswertung verwendet werden dürfen.

ja $\square$ nein $\square$

Ich stimme zu, dass die personenbezogenen Daten, Untersuchungsergebnisse und Röntgenbilder aus der konsiliarischen Untersuchung für die Querschnittsstudie ausgewertet werden dürfen.

ja $\square$ nein $\square$

Ich erkläre hiermit mein Einverständnis zur Teilnahme an der geplanten Verlaufsstudie.

ja $\square$ nein $\square$ 
Für Praxispatienten: Ich entbinde meinen behandelnden Arzt (Dr. Rühlmann) von der Schweigepflicht gegenüber den Studienärzten der Poliklinik für Präventive Zahnmedizin, Parodontologie und Kariologie der Universitätsmedizin Göttingen.

ja $\square$ nein $\square$

Ich möchte die in der Studie festgestellten Untersuchungsergebnisse mitgeteilt bekommen?

ja $\square$ nein $\square$

Ich habe eine Kopie der Patienteninformation und dieser Einwilligungserklärung erhalten und erkläre hiermit meine freiwillige Teilnahme an dieser klinischen Studie.

Ort und Datum

Unterschrift des Teilnehmers

Ort und Datum

Unterschrift des Prüfarztes 
PD. Dr. med. dent. Dirk Ziebolz, Telefon: 0551/39-22877; Universitätsmedizin Göttingen, GeorgAugust-Universität; Zentrum Zahn,- Mund- und Kieferheilkunde, Poliklinik für Präventive Zahnmedizin, Parodontologie und Kariologie; Robert-Koch-Str. 40, 37075 Göttingen

\section{Einverständniserklärung (Sorgeberechtigte)}

„Klinische Querschnittsstudie zur Beurteilung der Mundgesundheit und möglicher Dysfunktionen des stomatognathen Systems bei Kindern und Jugendlichen mit Juveniler Idiopathischer Arthritis“

Ich (Sorgeberechtigter), wurde von einem Arzt vollständig über Risiken, Wesen, Bedeutung und Tragweite sowie Durchführung der Studie (zum Zwecke der Forschung) mit dem Titel: „Klinische Querschnittsstudie zur Beurteilung der Mundgesundheit und möglicher Dysfunktionen des stomatognathen Systems bei Kindern und Jugendlichen mit Juveniler Idiopathischer Arthritis" aufgeklärt.

Mir ist bekannt, dass im Rahmen dieses Forschungsvorhabens personenbezogene Daten meines Kindes ausgewertet werden sollen. Die Daten werden in pseudonymisierter (verschlüsselter) Form aufgezeichnet und gespeichert. Die Daten werden für einen Zeitraum von 10 Jahren aufbewahrt, danach werden alle personenbezogenen Daten gelöscht. Die personenbezogenen Daten werden nicht an Dritte weitergegeben.

Ich weiß, dass ich mein Einverständnis zur Teilnahme an der Studie jederzeit widerrufen kann. Im Falle des Widerrufs werden alle personenbezogenen Daten gelöscht.

Ich hatte ausreichend Zeit, mich zur Teilnahme meines Kindes an dieser Studie zu entscheiden und weiß, dass die Teilnahme freiwillig ist. Alle Fragen wurden zu meiner Zufriedenheit beantwortet.

Mir ist bekannt, dass ich jederzeit und ohne Angaben von Gründen diese Zustimmung widerrufen kann, ohne dass sich dieser Entschluss nachteilig auf die weitere Behandlung meines Kindes auswirkt. Ich bin damit einverstanden, dass die personenbezogenen Daten auch nach meinem Widerruf weiter für die Auswertung verwendet werden dürfen.

ja $\square$ nein $\square$

Ich stimme zu, dass die personenbezogenen Daten, Untersuchungsergebnisse und Röntgenbilder meines Kindes aus der konsiliarischen Untersuchung für die Querschnittsstudie ausgewertet werden dürfen

ja $\square$ nein $\square$

Ich erkläre hiermit mein Einverständnis zur Teilnahme meines Kindes an der geplanten Verlaufsstudie. 
ja $\square$ nein $\square$

Für Praxispatienten: Ich entbinde meinen behandelnden Arzt (Dr. Rühlmann)von der Schweigepflicht gegenüber den Studienärzten der Poliklinik für Präventive Zahnmedizin, Parodontologie und Kariologie der Universitätsmedizin Göttingen.

ja $\square$ nein $\square$

Ich möchte die in der Studie festgestellten Untersuchungsergebnisse mitgeteilt bekommen?

ja $\square$ nein $\square$

Ich habe eine Kopie der Patienteninformation und dieser Einwilligungserklärung erhalten und erkläre hiermit meine freiwillige Teilnahme an dieser klinischen Studie.

Ort und Datum

Unterschrift des Teilnehmers

und / oder Sorgeberechtigte

Ort und Datum

Unterschrift des Prüfarztes 


\subsection{Untersuchungsblatt RDC/TMD}

\section{Untersuchungsblatt (Achse I Befunde)}

Alter:

Geschlecht:

männlich 0 口

weiblich 1 व

1. Haben Sie Schmerzen in der rechten Gesichts-

keine

rechts

$0 \square$

hälfte, in der linken oder in beiden?

links

1 ㅁ

beide

2 ㅁ

3 口

2. Können Sie auf die schmerzende Stelle zeigen?

(Der Untersucher tastet die gezeigte Stelle $a b$, wenn unklar ist, ob es Muskel- oder Gelenkschmerzen sind)

rechts

$\begin{array}{ll}\text { keine } & 0 \square \\ \text { Kiefergelenk } & 1 \text { ㅁ } \\ \text { Muskel } & 2 \text { 口 } \\ \text { beides } & 3 \square\end{array}$

links

keine

Kiefergelenk $1 \square$

Muskel

$2 \square$

beides

3 무

\section{Mundöffinungsbewegung}

gerade
seitliche Abweichung (Deflexion) nach rechts
"s $\mathrm{s}$-formige Abweichung (Deviation) nach rechts
seitliche Abweichung (Deflexion) nach links
"s"-formige Abweichung (Deviation) nach links
anderes Muster

Typ

4. Vertikaler Bewegungsumfang
a) akt. max. Öffnung ohne Schmerzen $\mathrm{mm}$
b) akt. max. Öffnung
c) passive max. Öffinung $\mathrm{mm}$
d) Overbite $\mathrm{mm}$
e) Overjet $\mathrm{mm}$ $\mathrm{mm}$

Muskelschmerzen

b) c)

keine $0 \square \quad 0 \square$

rechts $1 \square \quad 1 \square$

links $2 \square 2 \square$

beide $3 \square 3 \square$
Gelenkschmerzen

b) c)

keine $0 \square 0 \square$

rechts $1 \square \quad 1 \square$

links 2 口 2 口

beide $3 \square 3 \square$ 
5. Gelenkgeräusche (Palpation)

\begin{tabular}{|c|c|c|c|c|}
\hline a) Öffnung & $\begin{array}{l}\text { keine } \\
\text { Knacken } \\
\text { starkes Reiben } \\
\text { feines Reiben } \\
\text { Öffnungsknacken bei }\end{array}$ & & 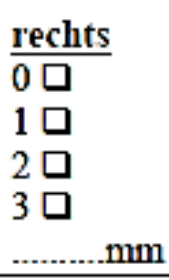 & $\begin{array}{r}\frac{\text { links }}{0 \square} \\
1 \square \\
2 \square \\
3 \mathrm{r} \\
\end{array}$ \\
\hline \multicolumn{5}{|l|}{ b) Schließen } \\
\hline & $\begin{array}{l}\text { keine } \\
\text { Knacken } \\
\text { starkes Reiben } \\
\text { feines Reiben } \\
\text { Schließungsknacken bei }\end{array}$ & & $\begin{array}{l}0 \square \\
1 \square \\
2 \square \\
3 \square \\
\text {..........mm }\end{array}$ & $\begin{array}{r}0 \square \\
1 \square \\
2 \square \\
3 \mathrm{~m} \\
\mathrm{~m}\end{array}$ \\
\hline c) reziprokes & $\begin{array}{l}\text { Knacken } \\
\text { verhindert bei } \\
\text { protrusiver Öffnung }\end{array}$ & $\begin{array}{l}\text { nein } \\
\text { ja } \\
\mathrm{NZ}\end{array}$ & $\begin{array}{l}\text { rechts } \\
0 \square \\
1 \square \\
9 \square\end{array}$ & $\begin{array}{l}\text { links } \\
0 \square \\
1 \square \\
9 \square\end{array}$ \\
\hline
\end{tabular}

\section{Bewegungen}

\begin{tabular}{|c|c|c|c|c|c|c|c|c|c|}
\hline \multirow[t]{2}{*}{ Bewegung } & \multirow{2}{*}{$\begin{array}{l}\text { Bewegung } \\
\text { in } \mathrm{mm}\end{array}$} & \multicolumn{4}{|c|}{ Muskelschmerzen } & \multicolumn{4}{|c|}{ Gelenkschmerzen } \\
\hline & & $\begin{array}{c}\text { keine } \\
0\end{array}$ & $\begin{array}{c}\text { rechts } \\
1\end{array}$ & $\begin{array}{c}\text { links } \\
2\end{array}$ & $\begin{array}{c}\text { beide } \\
3\end{array}$ & $\begin{array}{c}\text { keine } \\
0\end{array}$ & \begin{tabular}{|c} 
rechts \\
1
\end{tabular} & $\begin{array}{c}\text { links } \\
2\end{array}$ & $\begin{array}{c}\text { beide } \\
3\end{array}$ \\
\hline \multicolumn{10}{|l|}{ Laterotr.rechts } \\
\hline \multicolumn{10}{|l|}{ Laterotr.links } \\
\hline Protrusion & & & & & & & & & \\
\hline Mittellinienabr & chung & & $\mathrm{mm}$ & & & $\begin{array}{l}\square \\
\square\end{array}$ & & & \\
\hline
\end{tabular}

7. Gelenkgeräusche bei Bewegung

Geräusche rechts $\quad$ Gerāusche links

\begin{tabular}{|c|c|c|c|}
\hline Bewegung nach rechts & $\begin{array}{l}\text { keine } \\
\text { Knacken } \\
\text { starkes Reiben } \\
\text { feines Reiben }\end{array}$ & $\begin{array}{l}00 \\
1 \text { 口 } \\
2 \text { 口 } \\
3 \text { 口 }\end{array}$ & $\begin{array}{l}0 \square \\
1 \square \\
2 \square \\
3 \square\end{array}$ \\
\hline Bewegung nach links & $\begin{array}{l}\text { keine } \\
\text { Knacken } \\
\text { starkes Reiben } \\
\text { feines Reiben }\end{array}$ & $\begin{array}{l}0 \text { 口 } \\
1 \text { 口 } \\
2 \text { 口 } \\
3 \text { 口 }\end{array}$ & $\begin{array}{l}0 \square \\
1 \text { 口 } \\
2 \text { 口 } \\
3 \text { 口 }\end{array}$ \\
\hline Protrusion & $\begin{array}{l}\text { keine } \\
\text { Knacken } \\
\text { starkes Reiben } \\
\text { feines Reiben }\end{array}$ & $\begin{array}{l}0 \text { 口 } \\
1 \text { 口 } \\
2 \text { 口 } \\
3 \text { 口 }\end{array}$ & $\begin{array}{l}0 \square \\
1 \text { 口 } \\
2 \square \\
3 \square\end{array}$ \\
\hline
\end{tabular}


8. Extraorale Muskelpalpation

kein Schmerz/nur Druck $=0$

leichter Schmerz

$=1$

mäBiger Schmerz

heftiger Schmerz

$=2$

$=3$

a) Temporalis

links

posteriorer Teil

0 무

2 ㅁ 2 口

$\begin{array}{lll} & 3 \square & 3 \square \\ \text { b) Temporalis } & 0 \square & 0 \square\end{array}$

medialer Teil 1 口 $1 \square$

2 口 2 口

$\begin{array}{lll} & 3 \square & 3 \square \\ \text { c) Temporalis } & 0 \square & 0 \square\end{array}$

anteriorer Teil 1 口 1 口

2 口 2口

\begin{tabular}{lll} 
& $3 \square$ & $3 \square$ \\
\hline d) Masseterursprung & $0 \square$ & $0 \square$
\end{tabular}

1 口 1 口

2 口 2 口

\begin{tabular}{lll} 
& $3 \square$ & $3 \square$ \\
\hline e) Masseterkörper & $0 \square$ & $0 \square$
\end{tabular}

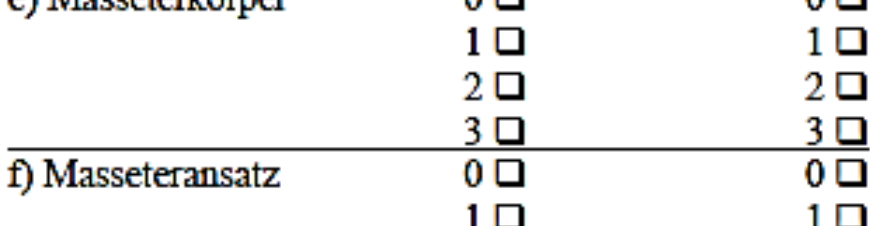

\begin{tabular}{ll}
$2 \square$ & $2 \square$ \\
$3 \square$ & $3 \square$ \\
\hline
\end{tabular}

\begin{tabular}{lll}
\hline g) Regio retromandibularis & $0 \mathrm{D}$ & $0 \mathrm{Q}$ \\
& $1 \mathrm{a}$ & $1 \mathrm{Q}$
\end{tabular}

2 口 2 口

3 3 口

h) Regio submandibularis 0 口 10

1 는

2 口 2 口

9. Palpation des Gelenkes

a) lateraler Kondylenpol $0 \mathrm{a}$

1 口 1 व

\begin{tabular}{lll} 
& 20 & $2 \square$ \\
& $3 \square$ & $3 \square$ \\
\hline b) posteriorer Kondylenpol & $0 \square$ & $0 \square$
\end{tabular}

1 口 1 व

2 口 2 口

10. intraorale Palpation

\begin{tabular}{lll} 
& $2 \square$ & $2 \square$ \\
\hline a) Pterygoideus lateralis & $3 \square$ & $3 \square$ \\
& $1 \square$ & $0 \square$ \\
& $2 \square$ & $1 \square$ \\
& $3 \square$ & $2 \square$ \\
b) Temporalissehne & $0 \square$ & $3 \square$ \\
& $1 \square$ & $0 \square$ \\
& $2 \square$ & $1 \square$ \\
& $3 \square$ & $2 \square$ \\
\hline
\end{tabular}

Datum:

Untersucher: 


\section{Literaturverzeichnis}

Abramowicz S, Kim S, Susarla HK, Kaban LB (2013): Differentiating arthritic from myofascial pain in children with juvenile idiopathic arthritis: preliminary report. J Oral Maxillofac Surg 71, 493-496

Ahmed N, Bloch-Zupan A, Murray KJ, Calvert M, Roberts GJ, Lucas VS (2004): Oral health of children with juvenile idiopathic arthritis. J Rheumatol $\underline{31}$, 1639-1643

Ainamo J, Barmes D, Beagrie G, Cutress T, Martin J, Sardo-Infirri J (1982): Development of the World Health Organization (WHO) community periodontal index of treatment needs (CPITN). Int Dent J $\underline{32}, 281-291$

Alessi S, Depaoli R, Canepari M, Bartolucci F, Zacchino M, Draghi F (2012): Baker's cyst in pediatric patients: Ultrasonographic characteristics. J Ultrasound $15,76-81$

Alkady EA, Helmy HA, Mohamed-Hussein AA (2012): Assessment of cardiac and pulmonary function in children with juvenile idiopathic arthritis. Rheumatol Int $\underline{32}, 39-46$

Arabshahi B, Cron RQ (2006): Temporomandibular joint arthritis in juvenile idiopathic arthritis: the forgotten joint. Curr Opin Rheumatol 18, 490-495

Argyropoulou MI, Margariti PN, Karali A, Astrakas L, Alfandaki S, Kosta P, Siamopoulou A (2009): Temporomandibular joint involvement in juvenile idiopathic arthritis: clinical predictors of magnetic resonance imaging signs. Eur Radiol $\underline{19}, 693-700$

Armitage GC (1999): Development of classification system for periodontal diseases and conditions. Ann Periodontol 4, 1-6

Arunachalam LT (2014): Autoimmune correlation of rheumatoid arthritis and periodontitis. J Indian Soc Periodontol 18, 666-669

Arvidsson LZ, Flatø B, Larheim TA (2009): Radiographic TMJ abnormalities in patients with juvenile idiopathic arthritis followed for 27 years. Oral Surg Oral Med Oral Pathol Oral Radiol Endod 108, 114-123

Barr T, Carmichael NM, Sándor GK (2008): Juvenile idiopathic arthritis: a chronic pediatric musculoskeletal condition with significant orofacial manifestations. J Can Dent Assoc 74, 813-821

Berntson L, Damgård M, Andersson-Gäre B, Herlin T, Nielsen S, Nordal E, Rygg M, Zak M, Fasth A (2008): HLA-B27 predicts a more extended disease with increasing age at onset in boys with juvenile idiopathic arthritis. J Rheumatol 35, 2055-2061

Beukelman T, Ringold S, Davis TE, DeWitt EM, Pelajo CF, Weiss PF, Kimura Y (2012): Disease-modifying antirheumatic drug use in the treatment of juvenile idiopathic arthritis: a cross-sectional analysis of the CARRA Registry. J Rheumatol $\underline{39}$, 1867-1874

Bhatt KH, Karjodkar FR, Sansare K, Patil D (2014): Juvenile idiopathic arthritis. Contemp Clin Dent $\underline{5}, 89-91$ 
Białowąs K, Swierkot J, Radwan-Oczko M (2014): [Role of Porphyromonas gingivalis in rheumatoid arthritis and inflammatory spondyloarthropathies]. Postepy Hig Med Dosw (Online) $\underline{68}$, 1171-1179

Billiau AD, Hu Y, Verdonck A, Carels C, Wouters C (2007): Temporomandibular joint arthritis in juvenile idiopathic arthritis: prevalence, clinical and radiological signs, and relation to dentofacial morphology. J Rheumatol 34, 19251933

Calderaro DC, Corrêa JD, Ferreira GA, Barbosa IG, Martins CC, Silva TA, Teixeira AL (2017): Influence of periodontal treatment on rheumatoid arthritis: a systematic review and meta-analysis. Rev Bras Reumatol Engl Ed 57, 238-244

Cannizzaro E, Schroeder S, Müller LM, Kellenberger CJ, Saurenmann RK (2011): Temporomandibular joint involvement in children with juvenile idiopathic arthritis. J Rheumatol $\underline{38}, 510-515$

Carlens C, Jacobsson L, Brandt L, Cnattingius S, Stephansson O, Askling J (2009): Perinatal characteristics, early life infections and later risk of rheumatoid arthritis and juvenile idiopathic arthritis. Ann Rheum Dis 68, 11591164

Carrasco R (2015): Juvenile idiopathic arthritis overview and involvement of the temporomandibular joint: prevalence, systemic therapy. Oral Maxillofac Surg Clin North Am 르, 1-10

Carvalho RT, Braga FS, Brito F, Capelli Junior J, Figueredo CM, Sztajnbok FR (2012): Temporomandibular joint alterations and their orofacial complications in patients with juvenile idiopathic arthritis. Rev Bras Reumatol $\underline{52}$, 907-911

Cedströmer AL, Andlin-Sobocki A, Berntson L, Hedenberg-Magnusson B, Dahlström L (2013): Temporomandibular signs, symptoms, joint alterations and disease activity in juvenile idiopathic arthritis - an observational study. Pediatr Rheumatol Online J 11, 37

Cedströmer AL, Ahlqwist M, Andlin-Sobocki A, Berntson L, HedenbergMagnusson B, Dahlström L (2014): Temporomandibular condylar alterations in juvenile idiopathic arthritis most common in longitudinally severe disease despite medical treatment. Pediatr Rheumatol Online J $\underline{12}, 43$

Celiker R, Gökçe-Kutsal Y, Eryilmaz M (1995): Temporomandibular joint involvement in rheumatoid arthritis. Relationship with disease activity. Scand $J$ Rheumatol 24, 22-25

Consolaro A, Ruperto N, Filocamo G, Lanni S, Bracciolini G, Garrone M, Scala S, Villa L, Silvestri G, Tani D et. al. (2012): Seeking insights into the Epidemiology, treatment and Outcome of Childhood Arthritis through a multinational collaborative effort: Introduction of the EPOCA study. Pediatr Rheumatol Online J $\underline{10}, 39$

Dāvidsone Z, Eglīte J, Lazareva A, Dzelzīte S, Šantere R, Bērziṇa D, Staṇēviča V (2016): HLA II class alleles in juvenile idiopathic arthritis patients with and without temporomandibular joint arthritis. Pediatr Rheumatol Online J $\underline{14}, 24$ 
Deschner J: Interaktionen zwischen Parodontitis und systemischen Erkrankungen. In: Stein JM (Hrsg.): Moderne Parodontologie in der Praxis. Band 1: Grundlagen, Klassifikation und Diagnostik. Spitta, Balingen 2011, 79-99

Detert J, Pischon N, Burmester GR, Buttgereit F (2010): Pathogenesis of parodontitis in rheumatic diseases. Z Rheumatol $\underline{69}, 109-112$

Deutsche Rheuma-Liga Bundesverband (Hrsg.): Kiefergelenkbeteiligung bei juveniler idiopathischer Arthritis. 2. Auflage; Deutscher Rheuma-Liga Bundesverband, Bonn 2016

Dev YP, Khuller N, Basavaraj P, Suresh G (2013): Rheumatoid Arthritis among Periodontitis Patients in Baddi Industrial Estate of Himachal Pradesh, India: A Cross Sectional Study. J Clin Diagn Res ㄱ, 2334-2337

Dick T: Labordiagnostik der juvenilen chronischen Arthritis. In: Venbrocks R. (Hrsg.): Neuroorthopädie und Rheumaorthopädie des Kindes (Praktische Orthopädie). Steinkopff-Verlag, Heidelberg 2000, 103-110

Dworkin SF, LeResche L (1992): Research diagnostic criteria for temporomandibular disorders: review, criteria, examinations and specifications, critique. J Craniomandib Disord $\underline{6}, 301-355$

Ellis JA, Munro JE, Chavez RA, Gordon L, Joo JE, Akikusa JD, Allen RC, Ponsonby AL, Craig JM, Saffery R (2012a): Genome-scale case-control analysis of CD4+ T-cell DNA methylation in juvenile idiopathic arthritis reveals potential targets involved in disease. Clin Epigenetics 4, 20

Ellis JA, Ponsonby AL, Pezic A, Chavez RA, Allen RC, Akikusa JD, Munro JE (2012b): CLARITY - ChiLdhood Arthritis Risk factor Identification sTudY. Pediatr Rheumatol Online J $\underline{10}, 37$

El-Shinnawi U, Soory M (2013): Associations between periodontitis and systemic inflammatory diseases: response to treatment. Recent Pat Endocr Metab Immune Drug Discov $\underline{7}, 169-188$

Emery P, Keystone E, Tony HP, Cantagrel A, van Vollenhoven R, Sanchez A, Alecock E, Lee J, Kremer J (2008): IL-6 receptor inhibition with tocilizumab improves treatment outcomes in patients with rheumatoid arthritis refractory to anti-tumour necrosis factor biologicals: results from a 24-week multicentre randomised placebo-controlled trial. Ann Rheum Dis $\underline{67}, 1516-1523$

Engelmann R, Müller-Hilke B (2009): Antibodies against citrullinated peptides in clinical practice and research. Z Rheumatol $\underline{68}, 485-490$

Erciyas K, Sezer U, Ustün K, Pehlivan Y, Kisacik B, Senyurt SZ, Tarakçioğlu M, Onat AM (2013): Effects of periodontal therapy on disease activity and systemic inflammation in rheumatoid arthritis patients. Oral Dis $\underline{19}$, 394-400

Fabri GM, Savioli C, Siqueira JT, Campos LM, Bonfá E, Silva CA (2014): Periodontal disease in pediatric rheumatic diseases. Rev Bras Reumatol 54, 311317

Feres de Melo AR, Ferreira de Souza A, de Oliveira Perestrelo B, Leite MF (2014): Clinical oral and salivary parameters of children with juvenile idiopathic arthritis. Oral Surg Oral Med Oral Pathol Oral Radiol 117, 75-80 
Fernandez-Viña M, Fink CW, Stastny P (1994): HLA associations in juvenile arthritis. Clin Exp Rheumatol 12, 205-214

Foeldvari I, Wierk A (2010): Effectiveness of leflunomide in patients with juvenile idiopathic arthritis in clinical practice. J Rheumatol $\underline{37}$, 1763-1767

Goldsmith DP (2012): Pericarditis: sometimes an autoinflammatory disease? J Clin Rheumatol 18, 227-228

Güntsch A, Seltmann T, Klinger G: Ätiologie und Pathogenese parodontaler Erkrankungen. In: Stein JM (Hrsg.): Moderne Parodontologie in der Praxis. Band 1: Grundlagen, Klassifikation und Diagnostik. Spitta, Balingen 2011, 29-77

Gustafsson BE, Quensel CE, Lanke LS, Lundqvist C, Grahen H, Bonow BE, Krasse B (1954): The Vipeholm dental caries study; the effect of different levels of carbohydrate intake on caries activity in 436 individuals observed for five years. Acta Odontol Scand 11, 232-264

Hahn YS, Kim JG (2010): Pathogenesis and clinical manifestations of juvenile rheumatoid arthritis. Korean J Pediatr $\underline{53}$, 921-930

Harris JG, Kessler EA, Verbsky JW (2013): Update on the treatment of juvenile idiopathic arthritis. Curr Allergy Asthma Rep 13, 337-346

Havemose-Poulsen A, Westergaard J, Stoltze K, Skjødt H, Danneskiold-Samsøe B, Locht H, Bendtzen K, Holmstrup P (2006): Periodontal and hematological characteristics associated with aggressive periodontitis, juvenile idiopathic arthritis, and rheumatoid arthritis. J Periodontol 77 , 280-288

Heasman PA, Waterhouse PJ: Periodontal diseases in children. In: Welbury R, Duggal MS, Hosey MT: Paediatric Dentistry. 4. Auflage; Oxford University Press, Oxford 2012, 199-218

Helkimo M (1974): Studies on function and dysfunction of the masticatory system. II. Index for anamnestic and clinical dysfunction and occlusal state. Sven Tandlak Tidskr $\underline{67}, 101-121$

Hellwig E, Klimek J, Attin T: Einführung in die Zahnerhaltung. Prüfungswissen Kariologie, Endodontologie und Parodontologie. 5. überarb. u. erw. Auflage; Deutscher Ärzte-Verlag, Köln 2009

Heubner G, Grosche M, Gahr M (2002): Therapie der juvenilen idiopathischen Arthritis. Monatsschr Kinderheilkd 4 , 445-451

Hiz O, Ediz L, Okzan Y, Bora A (2012): Clinical and magnetic resonance imaging findings of the temporomandibular joint in patients with rheumatoid arthritis. $\mathrm{J}$ Clin Med Res 4, 323-331

Hofer M, Southwood TR (2002): Classification of childhood arthritis. Best Pract Res Clin Rheumatol 16, 379-396

Holmstrup P, Damgaard C, Olsen I, Klinge B, Flyvbjerg A, Nielsen CH, Hansen PR (2017): Comorbidity of periodontal disease: two sides of the same coin? An introduction for the clinician. J Oral Microbiol $\underline{9}, 1332710$

Hu Y, Billiau AD, Verdonck A, Wouters C, Carels C (2009): Variation in dentofacial morphology and occlusion in juvenile idiopathic arthritis subjects: a casecontrol study. Eur J Orthod $\underline{31}, 51-58$ 
Huang JL (2012): New advances in juvenile idiopathic arthritis. Chang Gung Med J $\underline{35}, 1-14$

Hucke K: Pilotstudie zum Zahnfleischstatus bei Patienten/innen mit juveniler idiopathischer Arthritis. Med. Diss. Berlin 2014

Isenberg DA, Maddison PJ, Woo P, Glass D, Breedveld FC: Oxford textbook of rheumatology, 3. Auflage; Oxford University Press, Oxford 2004.

Jaakkola JJ, Gissler M (2005): Maternal smoking in pregnancy as a determinant of rheumatoid arthritis and other inflammatory polyarthropathies during the first 7 years of life. Int J Epidemiol 34, 664-671

Javed F, Ahmed HB, Mikami T, Almas K, Romanos GE, Al-Hezaimi K (2014): Cytokine profile in the gingival crevicular fluid of rheumatoid arthritis patients with chronic periodontitis. J Investig Clin Dent $\underline{5}, 1-8$

John MT, Hirsch C, Reiber T, Dworkin SF (2006): Translating the research diagnostic criteria for temporomandibular disorders into German: evaluation of content and process. J Orofac Pain 20, 43-52

Jordan A, Micheelis W (Hrsg.): Fünfte Deutsche Mundgesundheitsstudie - (DMS V). Deutscher Zahnärzte Verlag DÄV, Köln 2016.

Jordan AR, Becker N, Johren H-P, Zimmer S (2016): Early Childhood Caries and Caries Experience in Permanent Dentition: A 15-year Cohort Study. Swiss Dent J $\underline{126}, 114-119$

Kadavath S, Efthimiou P (2015): Adult-onset Still's disease-pathogenesis, clinical manifestations, and new treatment options. Ann Med 47, 6-14

Kahl-Nieke B (2013): The Forgotten Joint - Das Kiefergelenk bei Juveniler Idiopathischer Arthritis (JIA). Inf Orthod Kieferorthop 45, 221-226

Kahn P (2011): Juvenile idiopathic arthritis - an update on pharmacotherapy. Bull NYU Hosp Jt Dis $\underline{69}$, 264-276

Kanaparthy R, Kanaparthy A, Mahendra M (2012): C-reactive protein as a marker of periodontal disease. Gen Dent $\underline{60}, 1-5$

Kandilakis M, Lang NP: Plaqueinduzierte gingivale Erkrankungen. In: Lang NP (Hrsg.): Parodontalerkrankungen. Klassifikation und Charakterisierung. Quintessenz, Berlin 2003, 17-31

Karhulahti T, Ylijoki H, Rönning O (1993): Mandibular condyle lesions related to age at onset and subtypes of juvenile rheumatoid arthritis in 15-year-old children. Scand J Dent Res 101, 332-338

Kaslick RS, West TL, Chasens AI, Terasaki PI, Lazzara R, Weinberg S (1975): Association between HL-A2 antigen and various periodontal diseases in young adults. J Dent Res $\underline{54}, 424$

Kaur S, White S, Bartold PM (2013): Periodontal disease and rheumatoid arthritis: a systematic review. J Dent Res $\underline{92}$, 399-408

Kavanaugh A, Tomar R, Reveille J, Solomon DH, Homburger HA (2000): Guidelines for clinical use of the antinuclear antibody test and tests for specific autoantibodies to nuclear antigens. American College of Pathologists. Arch Pathol Lab Med 124, 71-81 
Keller H, Müller LM, Markic G, Schraner T, Kellenberger CJ, Saurenmann RK (2015): Is early TMJ involvement in children with juvenile idiopathic arthritis clinically detectable? Clinical examination of the TMJ in comparison with contrast enhanced MRI in patients with juvenile idiopathic arthritis. Pediatr Rheumatol Online J $\underline{13}, 56$

Keyes PH (1962): Recent advances in dental caries research. Bacteriology, Bacteriological findings, and biological implications. Int dent J $\underline{12}, 443-464$

Kinane DF, Peterson M, Stathopoulou PG (2006): Environmental and other modifying factors of the periodontal diseases. Periodontol $2000 \underline{40}, 107-119$

Kinderklinik Garmisch-Partenkirchen gGmbH - Deutsches Zentrum für Kinder- und Jugendrheumatologie (Hrsg.): Kinder- und Jugendrheuma - Wir können was tun. 2. Auflage; Kinderklinik Garmisch-Partenkirchen, GarmischPartenkirchen 2012

Kinloch A, Lundberg K, Wait R, Wegner N, Lim NH, Zendman AJ, Saxne T, Malmström V, Venables PJ (2008): Synovial fluid is a site of citrullination of autoantigens in inflammatory arthritis. Arthritis Rheum 58, 2287-2295

Kitai N, Kreiborg S, Murakami S, Bakke M, Møller E, Darvann TA, Takada K (2002): A three-dimensional method of visualizing the temporomandibular joint based on magnetic resonance imaging in a case of juvenile chronic arthritis. Int J Paediatr Dent $\underline{12}, 109-115$

Klein H, Palmer CE (1938): Studies on Dental Caries: V. Familial Resemblance in the Caries Experience of Siblings. Public Health Rep 53, 1353-1364

Klimm W: Kariologie. Ein Leitfaden für Studierende und Zahnärzte. Hanser, München 1997

Konig KG (1985): Kariogenität von zucker- und stärkehaltigen Nahrungsmitteln. Zahnarztl Prax $\underline{36}, 287-289$

Koos B, Fischer-Brandies H, Tzaribachev N (2011): Medikamente und Kieferorthopädie bei Kindern mit juveniler idiopathischer Arthritis. Arthritis Rheum (Munch.) $\underline{31}$, 410-414

Koos B, Twilt M, Kyank U, Fischer-Brandies H, Gassling V, Tzaribachev N (2014): Reliability of clinical symptoms in diagnosing temporomandibular joint arthritis in juvenile idiopathic arthritis. J Rheumatol $\underline{41}, 1871-1877$

Kumar P, Banik S (2013): Pharmacotherapy options in rheumatoid arthritis. Clin Med Insights Arthritis Musculoskelet Disord $\underline{6}$, 35-43

Landtwing K (1978): Evaluation of the normal range of vertical mandibular opening in children and adolescents with special reference to age and stature. $\mathrm{J} \mathrm{Ma-}$ xillofac Surg $\underline{6}, 157-162$

Lehmann RR: Ökologie der Mundhöhle. Grundlagen der Vorsorge. Thieme, Stuttgart 1991

Leksell E, Ernberg M, Magnusson B, Hedenberg-Magnusson B (2008): Intraoral condition in children with juvenile idiopathic arthritis compared to controls. Int J Paediatr Dent 18, 423-433

Lindroth AM, Park YJ (2013): Epigenetic biomarkers: a step forward for understanding periodontitis. J Periodontal Implant Sci $\underline{43}, 111-120$ 
Listgarten MA (1987): Nature of periodontal diseases: pathogenic mechanisms. J Periodont Res 22, 172-178.

Lucas VS, Roberts GJ (2005): Oro-dental health in children with chronic renal failure and after renal transplantation: a clinical review. Pediatr Nephrol $\underline{20}$, 1388-1394

Lyons H, Bernie J, Goldman HM (1959): Committee Reports...: Nomenclature and Classification Committee. J Periodontol 30, 74-77

Manger B (Hrsg.): Checkliste Rheumatologie (Checklisten der Medizin). 4. Auflage; Thieme, Stuttgart 2011

Manners PJ, Bower C (2002): Worldwide prevalence of juvenile arthritis why does it vary so much. J Rheumatol $\underline{29}, 1520-1530$

Marsh P, Martin MV: Orale Mikrobiologie. Thieme, Stuttgart 2003

Mason T, Rabinovich CE, Fredrickson DD, Amoroso K, Reed AM, Stein LD, Kredich DW (1995): Breast feeding and the development of juvenile rheumatoid arthritis. J Rheumatol 22, 1166-1170

Mayer Y, Balbir-Gurman A, Machtei EE (2009): Anti-tumor necrosis factor-alpha therapy and periodontal parameters in patients with rheumatoid arthritis. $\mathrm{J}$ Periodontol $\underline{80}, 1414-1420$

Meier FM, Frerix M, Hermann W, Müller-Ladner U (2013): Current immunotherapy in rheumatoid arthritis. Immunotherapy $\underline{5}$, 955-974

Mengel R, Flores-de-Jacoby L: Ätiologie und Pathogenese entzündlicher Erkrankungen. In: Mutschelknauss R (Hrsg.): Lehrbuch der klinischen Parodontologie. Quintessenz, Berlin 2000, 93-99

Micheelis E, Schroeder W: Risikogruppenstratifizierung bezüglich Karies (12jährige Kinder) und Parodontalerkrankungen (35- bis 44-jährige Erwachsene). In: Micheelis W, Schiffner U (Hrsg.): Vierte deutsche Mundgesundheitsstudie (DMS IV). Band 1. Deutscher Zahnärzte-Verlag DÄV, Köln 2006, 399-406

Mikuls TR, Payne JB, Yu F, Thiele GM, Reynolds RJ, Cannon GW, Markt J, McGowan D, Kerr GS, Redman RS et al. (2014): Periodontitis and Porphyromonas gingivalis in patients with rheumatoid arthritis. Arthritis Rheumatol $\underline{66}, 1090-1100$

Miller WD: Die Mikroorganismen der Mundhöhle. Die örtlichen und allgemeinen Erkrankungen, welche durch dieselben hervorgerufen werden. Thieme, Leipzig 1889.

Minden K (2009): Juvenile arthritis - clinical features and aspects of health care. Arthritis Rheum (Munch.) 29, 63-69

Miranda LA, Fischer RG, Sztajnbok FR, Figueredo CM, Gustafsson A (2003): Periodontal conditions in patients with juvenile idiopathic arthritis. J Clin Periodontol $\underline{30}, 969-974$

Mizuno N, Niitani M, Shiba H, Iwata T, Hayashi I, Kawaguchi H, Kurihara H (2011): Proteome analysis of proteins related to aggressive periodontitis combined with neutrophil chemotaxis dysfunction. J Clin Periodontol $\underline{38}, 310-317$ 
Müller HP: Parodontologie (Checklisten der Zahnmedizin). 3. aktualisierte Auflage; Thieme, Stuttgart 2012

Müller L, Kellenberger CJ, Cannizzaro E, Ettlin D, Schraner T, Bolt IB, Peltomäki T, Saurenmann RK (2009): Early diagnosis of temporomandibular joint involvement in juvenile idiopathic arthritis: a pilot study comparing clinical examination and ultrasound to magnetic resonance imaging. Rheumatology (Oxford) $\underline{48}, 680-685$

Neff D (1967): Acid production from different carbohydrate sources in human plaque in situ. Caries Res $1,78-87$

Neufeld KM, Karunanayake CP, Maenz LY, Rosenberg AM (2013): Stressful life events antedating chronic childhood arthritis. J Rheumatol $\underline{40}, 1756-1765$

Newbrun E (1969): Sucrose, the arch criminal of dental caries. ASDC J Dent Child $\underline{36}, 239-248$

Niibo P, Pruunsild C, Voog-Oras Ü, Nikopensius T, Jagomägi T, Saag M (2016): Contemporary management of TMJ involvement in JIA patients and its orofacial consequences. EPMA J $\underline{7}, 12$

Nimmrich S, Horneff G (2015): Incidence of herpes zoster infections in juvenile idiopathic arthritis patients. Rheumatol Int $\underline{35}$, 465-470

Nisihara RM, Skare T, Silva MB, Silva RM, Silva DJ (2009): Antinucleosome antibodies in juvenile chronic arthritis. Clin Rheumatol 28, 1461-1463

Nobre CM, Fernandes-Costa AN, de Melo Soares MS, Pugliesi DM, de Vasconcelos Gurgel BC (2016): Periodontal disease detection in primary and mixed dentitions. Eur Arch Paediatr Dent 17, 407-411

Oberle EJ, Harris JG, Verbsky JW (2014): Polyarticular juvenile idiopathic arthritis - epidemiology and management approaches. Clin Epidemiol $\underline{6}, 379-393$

Ogrendik M (2013): Rheumatoid arthritis is an autoimmune disease caused by periodontal pathogens. Int J Gen Med $\underline{6}$, 383-386

Omar A, Abo-Elyoun I, Hussein H, Nabih M, Atwa H, Gad S, Emad Y (2013): Anticyclic citrullinated peptide (anti-CCP) antibody in juvenile idiopathic arthritis (JIA): correlations with disease activity and severity of joint damage (a multicenter trial). Joint Bone Spine $\underline{80}$, 38-43

Omoyinmi E, Forabosco P, Hamaoui R, Bryant A, Hinks A, Ursu S, Wedderburn LR, Thomson W, Lewis CM, Woo P (2012): Association of the IL-10 gene family locus on chromosome 1 with juvenile idiopathic arthritis (JIA). PLoS ONE $\underline{7}$, e47673

Ortiz P, Bissada NF, Palomo L, Han YW, Al-Zahrani MS, Panneerselvam A, Askari A (2009): Periodontal therapy reduces the severity of active rheumatoid arthritis in patients treated with or without tumor necrosis factor inhibitors. $J$ Periodontol $\underline{80}$, 535-540

Page RC, Schroeder HE (1976): Pathogenesis of inflammatory periodontal disease. A summary of current work. Lab Invest $\underline{34}, 235-249$

Page RC, Kornman KS (1997): The pathogenesis of human periodontitis: an introduction. Periodontol $2000 \underline{14}$, 9-11 
Paris S, Meyer-Lückel H: Paradigmenwechsel. In: Meyer-Lückel H, Paris S, Ekstrand KR (Hrsg.): Karies. Wissenschaft und klinische Praxis. Thieme, Stuttgart 2012, 71-74

Pedersen TK, Küseler A, Gelineck J, Herlin T (2008): A prospective study of magnetic resonance and radiographic imaging in relation to symptoms and clinical findings of the temporomandibular joint in children with juvenile idiopathic arthritis. J Rheumatol $\underline{35}$, 1668-1675

Persson GR (2012): Rheumatoid arthritis and periodontitis- inflammatory and infectious connections. Review of the literature. J Oral Microbiol 4, 1-16

Peter KP, Mute BR, Pitale UM, Shetty S, Hc S, Satpute PS (2014): Prevalence of periodontal disease and characterization of its extent and severity in an adult population - an observational study. J Clin Diagn Res $\underline{8}$, ZC04-07

Ploski R, Vinje O, Rønningen KS, Spurkland A, Sørskaar D, Vartdal F, Førre O (1993): HLA class II alleles and heterogeneity of juvenile rheumatoid arthritis. DRB ${ }^{\star} 0101$ may define a novel subset of the disease. Arthritis Rheum $\underline{36}, 465-472$

Pohjankoski H, Kautiainen H, Korppi M, Savolainen A (2012): Simultaneous juvenile idiopathic arthritis and diabetes mellitus type 1 -- a Finnish nationwide study. J Rheumatol $\underline{39}$, 377-381

Prahalad S, Glass DN (2008): A comprehensive review of the genetics of juvenile idiopathic arthritis. Pediatr Rheumatol Online J $\underline{6}, 11$

Prahalad S, Martins TB, Tebo AE, Whiting A, Clifford B, Zeft AS, McNally B, Bohnsack JF, Hill HR (2008): Elevated serum levels of soluble CD154 in children with juvenile idiopathic arthritis. Pediatr Rheumatol Online J $\underline{6}, 8$

Prahalad S, Thompson SD, Conneely KN, Jiang Y, Leong T, Prozonic J, Brown MR, Ponder LA, Angeles-Han ST, Vogler LB et. al. (2012): Hierarchy of risk of childhood-onset rheumatoid arthritis conferred by HLA-DRB1 alleles encoding the shared epitope. Arthritis Rheum $\underline{64}, 925-930$

Prakken B, Albani S, Martini A (2011): Juvenile idiopathic arthritis. Lancet $\underline{377}$, 2138-2149

Raindi D (2016): Nutrition and Periodontal Disease. Dent Update $\underline{43}$, 66-68, 71-72

Ravelli A, Martini A (2007): Juvenile idiopathic arthritis. Lancet $\underline{369}$, 767-778

Reichert S, Machulla HK, Fuchs C, John V, Schaller HG, Stein J (2006): Is there a relationship between juvenile idiopathic arthritis and periodontitis? J Clin Periodontol $\underline{33}, 317-323$

Reichert S, Stein J, Fuchs C, John V, Schaller HG, Machulla HK (2007): Are there common human leucocyte antigen associations in juvenile idiopathic arthritis and periodontitis? J Clin Periodontol $\underline{34}$, 492-498

Reutter G, Girschick H: Juvenile idiopathische Arthritis. In: Gruber AA, DonhauserGruber U (Hrsg.): Rheuma - Untersuchen und Behandeln entzündlichrheumatischer Erkrankungen. Thieme, Stuttgart 2013, 61-75

Reutter-Simon G (2013): Rheuma - Was ist das?. Onlinequelle unter: https://www.yumpu.com/de/document/view/9547522/juvenile-idiopathischearthritis-jia-dr-med-gert-reutter-simon/3, letzter Zugriff am: 01.03.2016 
Reynolds MA (2014): Modifiable risk factors in periodontitis: at the intersection of aging and disease. Periodontol $2000 \underline{64}, 7-19$

Rohlin M, Petersson A (1989): Rheumatoid arthritis of the temporomandibular joint: radiologic evaluation based on standard reference films. Oral Surg Oral Med Oral Pathol 67, 594-599

Routsias JG, Goules JD, Goules A, Charalampakis G, Pikazis D (2011): Autopathogenic correlation of periodontitis and rheumatoid arthritis. Rheumatology (Oxford) $\underline{50}, 1189-1193$

Russo E, Trevisi E, Zulian F, Battaglia MA, Viel D, Facchin D, Chiusso A, Martinuzzi A (2012): Psychological profile in children and adolescents with severe course Juvenile Idiopathic Arthritis. ScientificWorldJournal 2012, 841375

Saurenmann R (2014): Clinical diagnosis of temporomandibular joint arthritis: a difficult task. J Rheumatol 41, 1734-1736

Sävendahl L (2012): The effect of acute and chronic stress on growth. Sci Signal $\underline{5}, \mathrm{pt} 9$

Savioli C, Silva CA, Ching LH, Campos LM, Prado EF, Siqueira JT (2004): Dental and facial characteristics of patients with juvenile idiopathic arthritis. Rev Hosp Clin Fac Med Sao Paulo 59, 93-98

Savolainen HA, Lehtimäki M, Kautiainen H, Aho K, Anttila P (1998): HLA B27: a prognostic factor in juvenile chronic arthritis. Clin Rheumatol 17, 121-124

Saxer UP, Mühlemann HR (1975): Motivation and education. SSO Schweiz Monatsschr Zahnheilkd 85, 905-919

Scardapane A, Breda L, Lucantoni M, Chiarelli F (2012): TNF-a Polymorphisms in Juvenile Idiopathic Arthritis: Which Potential Clinical Implications? Int J Rheumatol 2012, 756291

Schroeder HE: Pathobiologie oraler Strukturen. Zähne, Pulpa, Parodont. 3. überarb. Auflage; Karger, Basel 1997

Seguro LP, Rosario C, Shoenfeld Y (2013): Long-term complications of past glucocorticoid use. Autoimmun Rev $\underline{12}, 629-632$

Seymour RA (2003): Dentistry and the medically compromised patient. Surgeon 1 , 207-214

Shaw L, Glenwright HD (1989): The role of medications in dental caries formation: need for sugar-free medication for children. Pediatrician 16, 153-155

Siamopoulou A, Mavridis AK, Vasakos S, Benecos P, Tzioufas AG, Andonopoulos AP (1989): Sialochemistry in juvenile chronic arthritis. $\mathrm{Br} \mathrm{J}$ Rheumatol $\underline{28}$, 383-385

Socransky SS, Haffajee AD, Cugini MA, Smith C, Kent RL Jr (1998): Microbial complexes in subgingival plaque. J Clin Periodontol $\underline{25}$, 134-144

Stabrun AE, Larheim TA, Høyeraal HM (1989): Temporomandibular joint involvement in juvenile rheumatoid arthritis. Clinical diagnostic criteria. Scand $\mathrm{J}$ Rheumatol $\underline{18}, 197-204$ 
Stein SH, Borke JL, Cummings LA (1999): Effects of chronic adult periodontitis and endotoxin (LPS) on gingival fibroblast plasma membrane Ca++-pump. Connect Tissue Res $\underline{40}, 59-66$

Stock CJ, Ogilvie EM, Samuel JM, Fife M, Lewis CM, Woo P (2008): Comprehensive association study of genetic variants in the IL-1 gene family in systemic juvenile idiopathic arthritis. Genes Immun $\underline{9}$, 349-357

Stoll ML, Sharpe T, Beukelman T, Good J, Young D, Cron RQ (2012): Risk factors for temporomandibular joint arthritis in children with juvenile idiopathic arthritis. J Rheumatol 39, 1880-1887

Storhaug K, Holst D (1987): Caries experience of disabled school-age children. Community Dent Oral Epidemiol 15, 144-149

Straka M, Trapezanlidis M, Dzupa P, Pijak R (2012): Associations between marginal periodontitis and rheumatoid arthritis. Neuro Endocrinol Lett $\underline{33}, 16-20$

Sugiyama D, Nishimura K, Tamaki K, Tsuji G, Nakazawa T, Morinobu A, Kumagai $S$ (2010): Impact of smoking as a risk factor for developing rheumatoid arthritis: a meta-analysis of observational studies. Ann Rheum Dis $\underline{69}, 70-81$

Svensson B, Larsson A, Adell R (2001): The mandibular condyle in juvenile chronic arthritis patients with mandibular hypoplasia: a clinical and histological study. Int J Oral Maxillofac Surg $\underline{30}$, 300-305

Tanchyk AP (1991): Dental considerations for the patient with juvenile rheumatoid arthritis. Gen Dent 39, 330-332

Tang Q, Fu H, Qin B, Hu Z, Liu Y, Liang Y, Zhou L, Yang Z, Zhong R. (2017). A Possible Link Between Rheumatoid Arthritis and Periodontitis: Systematic Review and Meta-analysis. Int J Periodontics Restorative Dent 37, 79-86

Tebo AE, Jaskowski T, Davis KW, Whiting A, Clifford B, Zeft A, McNally B, Hill HR, Bohnsack J, Prahalad S (2012): Profiling anti-cyclic citrullinated peptide antibodies in patients with juvenile idiopathic arthritis. Pediatr Rheumatol Online J 10,29

Terasaki PI, Kaslick RS, West TL, Chasens Al (1975): Low HL-A2 frequency and periodontitis. Tissue Antigens $\underline{5}$, 286-288

Thomson W, Barrett JH, Donn R, Pepper L, Kennedy LJ, Ollier WE, Silman AJ, Woo P, Southwood T (2002): Juvenile idiopathic arthritis classified by the ILAR criteria: HLA associations in UK patients. Rheumatology (Oxford) 41, 1183-1189

Twilt M, Mobers SM, Arends LR, ten Cate R, van Suijlekom-Smit L (2004): Temporomandibular involvement in juvenile idiopathic arthritis. J Rheumatol $\underline{31}$, 1418-1422

Twilt M, Schulten AJ, Verschure F, Wisse L, Prahl-Andersen B, van SuijlekomSmit LW (2008): Long-term followup of temporomandibular joint involvement in juvenile idiopathic arthritis. Arthritis Rheum $\underline{59}, 546-552$

Tzaribachev N, Weber D, Horger M (2010): Juvenile idiopathic arthritis: the silent killer of pediatric temporomandibular joints. Z Rheumatol $\underline{69}$, 124-129 
Vahabi S, Rostamian A, Baniebrahimi G (2015): Characteristics and relationship of periodontal disease with juvenile idiopathic and rheumatoid arthritis. Dent Res J (Isfahan) 12, 541-547

Van der Velden U, Abbas F, Armand S, Loos BG, Timmerman MF, Van der Weijden GA, Van Winkelhoff AJ, Winkel EG (2006): Java project on periodontal diseases. The natural development of periodontitis: risk factors, risk predictors and risk determinants. J Clin Periodontol 33, 540-548

Van Dyke TE, Sheilesh D (2005): Risk factors for periodontitis. J Int Acad Periodontol $\underline{7}, 3-7$

von Koskull S, Truckenbrodt H, Holle R, Hörmann A (2001): Incidence and prevalence of juvenile arthritis in an urban population of southern Germany: a prospective study. Ann Rheum Dis $\underline{60}$, 940-945

Walton AG, Welbury RR, Foster HE, Thomason JM (1999): Juvenile chronic arthritis: a dental review. Oral Dis $\underline{5}, 68-75$

Walton AG, Welbury RR, Thomason JM, Foster HE (2000): Oral health and juvenile idiopathic arthritis: a review. Rheumatology (Oxford) $\underline{39}, 550-555$

Walton AG, Welbury RR, Foster HE, Wright WG, Thomason JM (2002): Sialochemistry in juvenile idiopathic arthritis. Oral Dis $\underline{8}, 287-290$

Weiss PF, Arabshahi B, Johnson A, Bilaniuk LT, Zarnow D, Cahill AM, Feudtner C, Cron RQ (2008): High prevalence of temporomandibular joint arthritis at disease onset in children with juvenile idiopathic arthritis, as detected by magnetic resonance imaging but not by ultrasound. Arthritis Rheum $\underline{58}$, 1189-1196

Welbury RR, Thomason JM, Fitzgerald JL, Steen IN, Marshall NJ, Foster HE (2003): Increased prevalence of dental caries and poor oral hygiene in juvenile idiopathic arthritis. Rheumatology (Oxford) $\underline{42}, 1445-1451$

Wentworth BA, Freitas-Neto CA, Foster CS (2014): Management of pediatric uveitis. F1000Prime Rep $\underline{6}, 41$

Wolf HF, Rateitschak EM, Rateitschak KH: Parodontologie (Farbatlanten der Zahnmedizin). 3. Auflage; Thieme, Stuttgart 2012

Woo P, Colbert RA (2009): An overview of genetics of paediatric rheumatic diseases. Best Pract Res Clin Rheumatol 23, 589-597

Yanagimachi M, Miyamae T, Naruto T, Hara T, Kikuchi M, Hara R, Imagawa T, Mori M, Kaneko T, Goto H et. al. (2011): Association of HLA-A*02:06 and HLA-DRB ${ }^{*} 04: 05$ with clinical subtypes of juvenile idiopathic arthritis. J Hum Genet $\underline{56}, 196-199$

Yoshinari N, Kameyama Y, Aoyama Y, Nishiyama H, Noguchi T (1994): Effect of long-term methotrexate-induced neutropenia on experimental periodontal lesion in rats. J Periodontal Res $\underline{29}$, 393-400

Young KA, Parrish LA, Zerbe GO, Rewers M, Deane KD, Michael Holers V, Norris JM (2007): Perinatal and early childhood risk factors associated with rheumatoid factor positivity in a healthy paediatric population. Ann Rheum Dis $\underline{66}, 179-183$ 


\section{Danksagung}

An dieser Stelle möchte ich allen, die mich bei der Durchführung dieser Arbeit unterstützt haben, herzlich danken.

Mein besonderer Dank gilt:

Dr. Sven Rinke für die Übernahme der Betreuung sowie die folgende effektive Zusammenarbeit bis zur Fertigstellung.

Prof. Dr. Dirk Ziebolz für die Überlassung und Betreuung dieser Doktorarbeit. Insbesondere für die stete fachliche Unterstützung und den konstruktiven, persönlichen Austausch bei allen mich bewegenden Fragen möchte ich mich herzlich bedanken.

Dr. Michael Rühlmann für die Bereitstellung von Informationen zum Thema juvenile idiopathische Arthritis.

Den Patienten von Dr. Michael Rühlmann und der Klinik für Kinder- und Jugendmedizin für ihre freiwillige Teilnahme an der Untersuchung.

Der Klinik für Kinder- und Jugendmedizin (unter der Leitung von Prof. Dr. med. Jutta Gärtner) sowie der Poliklinik für präventive Zahnmedizin, Parodontologie und Kariologie (unter der Leitung von Prof. Dr. Annette Wiegand) für die kooperative Bereitstellung benötigter Patientenunterlagen zur Auswertung. 